\author{
Maestría en Economía \\ Facultad de Ciencias Económicas \\ Universidad Nacional de La Plata
}

TESIS DE MAESTRIA

\begin{abstract}
ALUMNO
Matías Busso

TITULO

Un Análisis Extendido de la Discriminación Laboral por Género en Argentina

DIRECTOR

Leonardo Gasparini

FECHA DE DEFENSA
\end{abstract}

$12 / 23 / 2003$ 


\title{
Un Análisis Extendido de la Discriminación Laboral por Género en Argentina
}

\author{
Matías Busso \\ Tesis de Maestría \\ Maestría en Economía \\ Departamento de Economía \\ Universidad Nacional de La Plata
}

Director de Tesis: Dr. Leonardo Gasparini

La Plata, 26 de Diciembre de 2003 


\title{
Resumen
}

En este trabajo se analiza la existencia de discriminación por género en el mercado laboral argentino. En primer instancia se utiliza una descomposición de Oaxaca-Ransom para cuantificar e identificar las fuentes de discriminación salarial.

Adicionalmente, se utilizó una técnica novedosa que permite linealizar y luego descomponer un modelo probit con el objetivo de definir si existe discriminación en términos de la probabilidad de ser un trabajador informal y de ser un trabajador desempleado.

Se encontró que a pesar de que la brecha bruta de salarios ha presentado una tendencia decreciente, aún persiste cierto tipo de discriminación salarial en contra de las mujeres, aunque la misma es muy pequeña.

Por otra parte, la discriminación a través de la informalidad laboral y de la probabilidad de estar desempleado ha aumentado levemente, y la misma se mantiene positiva.

\begin{abstract}
In this paper we analyze the existence of gender discrimination in the Argentine labor market. We use the Oaxaca-Ransom decomposition to assess the quantity and source of wage discrimination by gender.

In addition, we use a new technique that first linearizes and then decompose a probit model to assess the existence of discrimination in employment probability and in the probability of being an informal worker.

We found that, although gender wage discrimination is declining in Argentina, at least some wage discrimination is still present in the labor market. More importantly, discrimination in terms of the probability of being an informal worker and on the probability of being unemployed is actually increasing.
\end{abstract}

Keywords: wage discrimination, gender discrimination, Argentina. 


\title{
Un Análisis Extendido de la Discriminación Laboral por Género en Argentina
}

\author{
Matías Busso $^{1}$ \\ Departamento de Economía \\ Universidad Nacional de La Plata
}

\section{Introducción}

La mayor parte de los trabajos sobre discriminación laboral realizados sobre Argentina coinciden en que la brecha de ingreso mensual entre hombres y mujeres es de alrededor del $30 \%$. Esto plantea un interrogante importante, ¿es esta brecha producto de la discriminación laboral en contra de las mujeres o es, en cambio, producto de diferencias en las características productivas de ambos grupos? Contestar esta pregunta es importante pues, al margen del problema ético subyacente, la existencia de discriminación es una de las fuentes de iniquidad distributiva. Si las mujeres, por el mero hecho de ser mujeres, no estuvieran en igualdad de oportunidades con relación a los hombres, se justificaría algún tipo de política económica tendiente a solucionar este problema.

El trabajo seminal de Becker "Economía de la Discriminación” de 1957, dio paso a una amplísima literatura cuyo principal objetivo es detectar, explicar y cuantificar la existencia de brechas "injustificadas" en el trato recibido por hombres y mujeres en el mercado laboral.

Oaxaca (1973) y Blinder (1973) contribuyeron de una manera fundamental a la medición de este tipo de eventos. Básicamente, puede pensarse que el salario percibido por cada trabajador depende de su dotación factorial (capital humano) y de la remuneración de mercado de esas dotaciones. Dado que en un contexto competitivo los factores se remuneran según su productividad marginal, toda diferencia en salarios que no esté explicada por diferencias en productividad podría calificarse como un fenómeno de

\footnotetext{
${ }^{1}$ Agradezco a Leo Gasparini por sus valiosos comentarios y colaboración a lo largo del trabajo. También a los participantes del Seminario de Tesis del Departamento de Economía de la Universidad Nacional de La Plata. Los errores remanentes son por supuesto mi responsabilidad exclusiva. Se agradecen comentarios a matiasb@umich.edu.
} 
discriminación. En otras palabras, puede decirse que existe discriminación siempre que se remunere distinto a un grupo de trabajadores con características iguales, o que se pague de manera similar a un conjunto de personas con características diferentes.

Ciertamente, podría argumentarse que el salario no es la única variable a través de la cual se discrimina a las mujeres. Muchas veces la discriminación está relacionada con la existencia de barreras a la entrada de trabajadoras mujeres a algunas actividades o a ciertos tipos de empresas. En otras ocasiones la discriminación puede tomar la forma de diferenciación en los tipos de tareas que se asignan a hombres y a mujeres, o de diferencias en la probabilidad de promoción dentro de la empresa. Cualquiera de estos fenómenos, sin embargo, tenderá a generar una caída en el salario relativo de las mujeres y, en consecuencia, será captado por la medida de discriminación salarial que se utilizará en este trabajo. Desde este punto de vista, analizar la discriminación a través de las diferencias salariales es un método bastante general de medir discriminación laboral.

Existen varios trabajos que documentan la existencia de discriminación salarial en distintos países del mundo.

Cain (1986) presenta un buen resumen de distintos resultados en materia de discriminación obtenidos para EEUU. Allí puede observarse claramente que cuando el ingreso se mide a partir del salario horario, la discriminación en contra de las mujeres varía en un rango que va del $15 \%$ al $40 \%$ dependiendo de la forma de medir productividad y del año analizado. Estas medidas tienden a aumentar considerablemente a valores que van del $20 \%$ al $60 \%$ cuando en lugar del salario horario se analizan las diferencias en el ingreso anual (lo que refleja diferencias en las horas trabajadas). Por otra parte Kunze (2000) encuentra resultados similares para los casos de Alemania y Gran Bretaña.

El fenómeno de discriminación por género también ha sido estudiado para el caso de varios países de Latinoamérica. Contreras y Galván (2001) hallan que en el caso de Bolivia durante los '90 ha persistido la discriminación por género (aunque la misma es menor para los individuos más jóvenes) variando en un rango que va del $30 \%$ al $40 \%$, 
aproximadamente. Asimismo, Loureiro (2001) muestra indicios de discriminación por género en Brasil; en tanto que Montenegro (2001) muestra para el caso de Chile la existencia de un diferencial sistemático en el retorno a la educación y experiencia de hombres y mujeres que se amplía para las personas ubicadas en los quintiles más altos de la distribución (condicional) del ingreso.

Para el caso argentino, Paz (2000) encuentra que en el año 1997 la discriminación resultó ser positiva pero baja (5\%) en relación con los resultados hallados para otros países. Este coeficiente está básicamente explicado por factores ligados al individuo y a su familia.

Este trabajo pretende avanzar en el análisis de la discriminación por género en Argentina básicamente en dos direcciones. En primer lugar, al igual que en los anteriores trabajos sobre discriminación realizados para Argentina y para otros países, se intentará detectar y cuantificar la existencia de discriminación salarial. Se pretende asimismo dar una idea de su evolución tanto en la Argentina como en su principal aglomerado urbano (el Gran Buenos Aires - GBA-), para lo cual es importante el cómputo de intervalos de confianza de las medidas de discriminación analizadas.

Ahora bien, bajo el supuesto de mercados competitivos donde no existen pagos extrasalariales al factor trabajo y los precios ajustan de manera instantánea ante desequilibrios de mercado, todo fenómeno de discriminación podría observarse a través de diferencias salariales. Sin embargo, el levantamiento de estos supuestos da lugar a la posible existencia de otras formas de discriminación en el mercado laboral que no son necesariamente captadas por la discriminación salarial. Un segundo objetivo entonces será analizar la posible existencia de discriminación en materia de formalidad laboral y de desempleo.

El resto del trabajo está ordenado de la siguiente manera. En la sección 2 se realiza un análisis descriptivo del comportamiento de las variables laborales enfatizando las diferencias por género y centrando la discusión en lo referido a la evolución en GBA y la estructura en Argentina. En la sección 3 se presenta y discute la metodología utilizada para 
medir discriminación y se discuten algunos supuestos que se hallan en los modelos sobre los cuales se apoyan estas metodologías. La sección 4 está destinada a exponer y analizar los principales resultados empíricos hallados brindando por un lado una idea de la evolución de la discriminación en GBA, y analizando para el caso argentino qué factores importan para explicar la discriminación, qué factores juegan a favor o en contra de los hombres y a favor o en contra de las mujeres. Finalmente, la sección 5 tiene por objetivo resumir los resultados hallados, dar algunos lineamientos de investigación futura y realizar algunos comentarios finales.

\section{Brechas laborales por género: un análisis descriptivo}

Este trabajo utiliza como fuente de información la Encuesta Permanente de Hogares (EPH) relevada en mayo y octubre de cada año por el Instituto Nacional de Estadística y Censos (INDEC) y diseñada especialmente para medir variables del mercado laboral y características demográficas y educativas de los individuos.

Más específicamente, en el caso de Argentina se trabajará con las encuestas de octubre de 1994 y octubre de 2000, acotándolas a individuos con edades entre 18 y 64 años. La elección de las edades mínima y máxima está relacionada con la edad a la cual se egresa de la escuela secundaria (en Argentina) y la edad a la cual los trabajadores tienen derecho a jubilarse, respectivamente. De esta forma la primer encuesta cuenta con 44.832 observaciones y la segunda con $34.947 .^{2}$

Asimismo, para analizar la evolución de la discriminación laboral se utilizaron las bases correspondientes al período 1985-2000 relevadas en octubre de cada año en el principal aglomerado urbano del país (Gran Buenos Aires). En promedio, cada base dispone de un total de 7.400 observaciones pertenecientes a personas entre 18 y 64 años. $^{3}$

\footnotetext{
${ }^{2}$ A lo largo de este trabajo, el aglomerado Argentina se refiere a un conjunto de 20 aglomerados urbanos los cuales se especifican en el Anexo 1.

${ }^{3}$ Aún así, debe notarse que existe una variabilidad importante en el número de observaciones de cada año (el desvío estándar es de 1.320 observaciones). Por otra parte, en el Anexo 1 se presenta un cuadro que detalla el significado y la construcción de las variables utilizadas a lo largo del trabajo.
} 
En el Cuadro 1 se presentan algunas estadísticas básicas. Puede observarse en primer lugar que las mujeres recibieron en promedio durante el año 2000, un ingreso mensual igual a un $74 \%$ del percibido por los hombres.

Debe notarse que este dato por si solo no constituye evidencia de que exista discriminación salarial en detrimento de las mujeres. Las mujeres podrían estar (por elección) en promedio menos capacitadas que los hombres, tener por lo tanto una menor productividad laboral y percibir desde luego un menor ingreso. Es importante hacer hincapié en la forma de interpretar los resultados de este trabajo. De aquí en más toda vez que se hable de la existencia de discriminación salarial, discriminación por desempleo o por informalidad se estará haciendo referencia a la existencia de una brecha en el salario (o en la tasa de desempleo o informalidad) que no puede ser explicada por las características observables de los individuos. Desde luego que esta medida es imperfecta. Existen una serie de características inobservables que generan diferencias observadas en ingreso, salario, tasa de desempleo e informalidad que no están relacionadas de manera alguna con la existencia de discriminación por género. Tómese por ejemplo el caso de diferencias en preferencias: si las mujeres, por ejemplo, prefieren trabajar en empleos con menor salario pero mayor remuneración no salarial (vgr. docencia), aún después de controlar por características observables, se observará una brecha salarial no explicada que no implica discriminación.

Ahora bien, la brecha entre hombres y mujeres observada en el ingreso mensual desaparece (de hecho se revierte) cuando se mira el salario horario. Una vez más, a priori podría pensarse que esta "brecha negativa" en el salario horario es un indicio de ausencia de discriminación salarial. Sin embargo, debe notarse que las mujeres tienen en promedio características productivas superiores a las de los hombres (Cuadro 2): en particular, poseen un nivel más alto de educación. En consecuencia, aún con un diferencial salarial negativo podría existir discriminación si el mercado no remunerase lo suficiente esas características para el caso de las mujeres o si remunerara de más a los hombres. Estaríamos entonces ante un caso de discriminación en el que el mercado remunera igual a dos grupos con características productivas diferentes. 
Por otra parte, debe tenerse en cuenta que cuando las personas reportan su ingreso en las encuestas de empleo suelen referirse a su salario de bolsillo. En un mercado en el que no existe intervención estatal, el salario de bolsillo es el salario pagado por la empresa y es todo lo recibido por el trabajador. Sin embargo, los trabajadores suelen recibir otros pagos adicionales: más específicamente, las personas, cuando son trabajadores formales, reciben distintos tipos de beneficios como obra social, indemnización y seguros de trabajo. Desde otro punto de vista, estos son costos cuasi-fijos (y hundidos) que en términos generales son solventados en parte por la empresa y en parte por el trabajador. ${ }^{4}$ En consecuencia las firmas podrían elegir la contratación de hombres en lugar de mujeres si es que les resultara costosa la potencial salida futura de la mujer del mercado laboral (que puede ocurrir, por ejemplo, ante un embarazo). También es posible que, al mismo tiempo, si a las mujeres les resultara difícil ingresar al mercado de trabajo, las trabajadoras discriminadas elijan ser contratadas informalmente como un mecanismo implícito para convencer a las firmas para que las contraten.

En ambos casos, los individuos pertenecientes al grupo discriminado enfrentarían una probabilidad mayor de ser informales y no gozarían de los beneficios asociados a la formalidad laboral. En este sentido, se puede observar también en el Cuadro 1 que las tasas de informalidad laboral ${ }^{5}$ resultaron, durante el año 2000, mayores en el caso de las mujeres lo que, si bien no demuestra la existencia de discriminación por informalidad, al menos justifica un análisis más exhaustivo.

Por último, si las rigideces del mercado laboral implican la imposibilidad de que la economía ajuste sus salarios para encontrar el equilibrio de mercado, entonces una parte de la discriminación no será captada por diferenciales salariales sino, en cambio, por diferenciales en la probabilidad de encontrar un empleo. Obsérvese que en este caso también, las tasas de desempleo femeninas son superiores a las masculinas y por lo tanto

\footnotetext{
${ }^{4}$ Quién pague realmente estos costos dependerá naturalmente de las elasticidades de oferta y demanda de trabajo.

$\mathrm{T}^{5}$ En este trabajo se define como trabajador informal a aquel que en el periodo de referencia declaró no gozar de jubilación o de otros beneficios (como por ejemplo, aguinaldo). Para una discusión detallada de la informalidad laboral en Argentina ver Gasparini (2000).
} 
también serán analizadas más profundamente. En particular, la conjetura es que las mujeres enfrentan una probabilidad de desempleo mayor que la enfrentada por los hombres.

En lo que sigue de esta sección se analizará por un lado, la evolución de estas brechas laborales (en términos de salarios, probabilidad de ser informal y probabilidad de ser desempleado) en el GBA ${ }^{6}$ entre 1985 y 2000 y, por el otro, los determinantes estructurales de las mismas.

\subsection{Un análisis de la tendencia en las brechas laborales}

La discriminación laboral en detrimento de las mujeres puede variar en el tiempo por ejemplo por cambios culturales o cambios económicos. Independientemente de las causas de esas variaciones, determinar el comportamiento tendencial es importante pues si se encontrara una tendencia creciente en la discriminación podrían comenzar a establecerse políticas públicas tendientes a detener ese crecimiento; si por el contrario se hallara una tendencia decreciente podría deducirse que el mero paso del tiempo estaría haciendo desaparecer la discriminación y, por ende, la intervención pública sería menos necesaria.

Como puede observarse en el Gráfico 1.A el ingreso mensual (tanto de los hombres como de las mujeres) presenta una tendencia decreciente en el último lustro de la década del '80, luego crece con la expansión económica de inicios de los '90 y finalmente cae nuevamente con el aumento del desempleo y el estancamiento de la economía.

Si bien el movimiento estilizado de la serie coincide entre sexos existe una brecha importante y persistente entre el ingreso mensual percibido por los hombres y el percibido por las mujeres. A lo largo del período las mujeres ganaron por mes en promedio un $66 \%$ de lo ganado por los hombres. Este resultado coincide con lo hallado por otros autores para

\footnotetext{
${ }^{6}$ La elección del aglomerado GBA para el análisis de la evolución de las diferentes variables se justifica por dos razones. En primer lugar, existen deficiencias informativas que no permiten un análisis de la evolución a nivel país a lo largo del tiempo (específicamente, para ciertos años no se relevaron algunos aglomerados). Dada esa limitación la elección del GBA se justifica por ser el mismo el aglomerado urbano más importante del país.
} 
el caso de EE.UU (Cain, 1986; Blau y Kahn, 2000), de Brasil (Loureiro, 2001) y de Argentina (Paz, 2000).

De todas formas si bien esta brecha parece alta, tiene también una tendencia a cerrarse. Mientras que en 1985 las mujeres ganaban un 62\% de los que ganaban los hombres, en el 2000 las mismas pasaron a percibir un salario mensual igual al 70\% del percibido por el sexo masculino.

Naturalmente, la brecha puede explicarse por la existencia de diferencias en el salario horario de hombres y mujeres o por diferencias en la participación del mercado de trabajo (o por ambos efectos conjuntamente).

En el Gráfico 1.B se observa de manera directa que el primer factor explicativo parece determinar, al menos en parte, el achicamiento de la brecha de ingresos mensuales. Si bien hasta 1990 los hombres venían recibiendo salarios horarios superiores a los de las mujeres (entre 1985 y 1990, las mismas ganaban en promedio un salario por hora igual al 85\% del ganado por los hombres) a partir de dicho año, esta diferencia se cerró súbitamente e inclusive llegó a revertirse.

La rapidez del cambio descartaría de antemano que el cierre de la brecha del salario horario se explicase por un cambio en las características productivas de las mujeres en relación a las de los hombres. Es difícil que en el muy corto plazo se adquiera o se destruya tanto capital humano como para cerrar una brecha del 15\% del salario horario. De esta manera una primera conjetura es que podría existir una reducción de la discriminación (si la misma existiese en un principio).

Para chequear preliminarmente esta hipótesis, en el Cuadro 3 se presenta la evolución de los años promedio de educación de hombres y mujeres. Nótese que las mujeres presentan niveles educativos más altos que los hombres. Más aún, esa diferencia tiende a ampliarse levemente con el paso del tiempo. Sin embargo no existe, como presuponíamos, ningún "salto" en el nivel educativo. 
Ahora bien, las reducciones de las brechas en el salario horario como en el ingreso mensual, coinciden con otros cambios bruscos acaecidos en el mercado laboral. En particular es un fenómeno muy documentado y conocido el incremento de la participación laboral femenina (Galiani, 2000) junto a una leve reducción en la intensidad de participación, expresada en horas trabajadas (Gasparini et al. 2002). Nótese que entre 1985 y 2000 la participación masculina permaneció relativamente estable en tanto que la femenina creció 17 puntos porcentuales (pasando del 40\% en 1985 al 57\% en 2000). Asimismo, el número de horas trabajadas por semana cayó fuertemente para los hombres y sólo levemente para las mujeres.

A pesar de estos cambios, persiste una importante diferencia a favor de los hombres tanto en las tasas de participación como en el número de horas trabajadas que explicaría la brecha existente en el ingreso mensual: trivialmente, a igual salario horario, si los hombres trabajan más que las mujeres percibirán un ingreso mensual superior. De manera que es posible que una parte importante de la brecha en el ingreso mensual no sea explicada por la existencia de discriminación sino por la elección de las mujeres de una menor participación en el mercado.

Por otra parte, el mencionado fenómeno de aumento de la participación laboral coincidió con un aumento en la tasa de desempleo tanto de las mujeres como de los hombres, tal como puede observarse en el Gráfico 2.a. Esto suele explicarse de la siguiente forma. A inicios de los '90 una gran cantidad de hombres sufrieron un shock negativo que les redujo el salario lo que incentivó a sus cónyuges a ingresar al mercado laboral para complementar ingresos de la familia. En un contexto de estancamiento de la demanda de trabajo, se incrementó la tasa de desempleo femenina. Nótese sin embargo que la tasa de desempleo femenina se incrementó en mayor medida que la masculina. Un análisis a realizar es, entonces, si un hombre y una mujer con características iguales enfrentaron en los '90 la misma probabilidad (condicional) de estar desempleados o si, en cambio, las mujeres sufrieron algún tipo de discriminación. 
Por último, en el Gráfico 2.b. se puede observar que además de la diferencia en materia de ingreso mensual, salario horario y desempleo, también existe una diferencia importante en las tasas de informalidad de hombres y mujeres. En particular, en casi todo el periodo bajo estudio la tasa de informalidad femenina superó a la masculina en un promedio de 4.8 puntos porcentuales. Esto también justifica analizar si parte de ese fenómeno se explica o no por la existencia de discriminación.

\subsection{Estructura de las brechas laborales en Argentina}

El análisis de la evolución de las brechas laborales (no condicionadas) debe ser complementado con un análisis de sus posibles determinantes. En particular, la existencia de las brechas por género en el ingreso, el salario horario, el desempleo y la informalidad, podrían simplemente estar justificadas por diferencias en las características productivas de hombres y mujeres y no por la presencia de un componente discriminatorio. El objetivo de esta sección es, en consecuencia, analizar si estas brechas se modifican cuando se condiciona en diferentes características sociodemográficas individuales que, a priori, podrían explicarlas. Este análisis se realizará ya no para el GBA sino para el caso argentino, con el fin de alcanzar una mayor generalidad de los resultados y en particular se analizarán dos años (1994 y 2000). Básicamente existen tres aspectos principales que pueden influir en la existencia de brechas en las variables laborales: factores ligados a la productividad (experiencia y educación), factores demográficos y otros factores inherentes a las industrias o las empresas.

Edad/Experiencia. El aumento de la edad de los individuos implica potencialmente dos efectos sobre su ingreso mensual (que puede definirse como el número de horas trabajadas en el mes multiplicado por el salario horario percibido). Por un lado, tiende a aumentar la participación en el mercado laboral y la intensidad del trabajo ${ }^{7}$ pues los individuos comienzan a salir del sistema educativo. Por otra parte, con el paso del tiempo los individuos irán ganando una mayor experiencia y por ende su salario horario también

\footnotetext{
${ }^{7}$ Se dice que una persona participa del mercado laboral cuando busca empleo o está trabajando. La intensidad del trabajo o de la participación, se refiere en cambio al número de horas trabajadas. La decisión de participar
} 
tenderá a aumentar. Dado que las mujeres presentan una participación cíclica en el mercado laboral $^{8}$ (Ehrenberg y Smith, 2000) y por ende acumulan un menor capital humano que los hombres, se espera encontrar un aumento de la brecha en el salario horario y por lo tanto en el ingreso mensual conforme se avanza hacia grupos etáreos más altos.

Las sospechas anteriores parecen de hecho estar confirmadas por los datos. En el Cuadro 4 se observa que el ingreso mensual crece con la edad pero el crecimiento es más fuerte en el caso de los hombres que en el de las mujeres, de modo que la brecha de ingresos mensuales se amplía conforme aumenta la edad de los individuos.

En 1994, las mujeres jóvenes ganaban por mes, en promedio, un $88 \%$ de lo que ganaban los hombres de su misma edad en tanto que las mujeres entre 44 y 64 años ganaban un $66 \%$ de lo percibido por hombres de ese grupo. Nótese que este fenómeno no parece estar explicado por diferencias en el salario horario que también presenta una brecha creciente con la edad, pero menor, o incluso favorable a las mujeres en las primeras etapas de la vida laboral. En cambio, están explicadas por importantes diferencias en participación e intensidad de participación: si bien ambas variables crecen con la edad en el caso de ambos sexos, el crecimiento en el caso de los hombres es superior al caso de las mujeres.

Un patrón similar se observa en materia de informalidad laboral: para el grupo de 19 a 24 años, en 2000 la tasa de informalidad de las mujeres es un 102\% de la de los hombres; estas diferencias se acentúan para los otros grupos etáreos (por ejemplo, la tasa de informalidad de las mujeres de 40 a 64 años es un $145 \%$ de la experimentada por los hombres del mismo grupo etáreo). En el caso de la tasa de desempleo la evidencia es menos clara: las diferencias son importantes en la primer etapa de la vida activa (19 a 39 años) y tienden a reducirse conforme se avanza a grupos de edades mayores.

es binaria (participa o no participa del mercado) en tanto que decisión de intensidad es (al menos hasta cierto punto) continua (trabaja $h$ horas por semana).

${ }^{8}$ La participación laboral femenina suele típicamente ser cíclica pues al inicio de la vida laboral la mujer entra al mercado, luego sale al momento de tener hijos y regresa una vez que los niños ingresan en edad escolar. 
Educación. Un segundo aspecto ligado a las características productivas de los individuos que resulta relevante a analizar, es la relación entre las brechas en las variables laborales y el nivel educativo de las personas (Cuadro 5). En principio, se observa que la brecha de ingresos mensuales se hace más pequeña a medida que aumenta el nivel de educación (esto puede verse más nítidamente en el año 2000 cuando las mujeres con nivel de educación primaria ganaban un 66\% de lo ganado por los hombres en tanto que para el grupo de educación superior la relación de ingresos aumentaba al 70\%). Es importante notar que este fenómeno está explicado por las decisiones de participación más que por el salario horario.

Más específicamente se halló que a mayores niveles educativos las diferencias en el salario horario que perciben hombres y mujeres tienden a aumentar (y no a reducirse como en el caso del ingreso mensual).

Sin embargo, al tiempo que en el caso de los hombres tanto la participación como las horas trabajadas permanecen relativamente constantes entre categorías educativas, para las mujeres ambos indicadores crecen fuertemente con la educación, reflejando probablemente el mayor costo de oportunidad de asignar tiempo a las tareas del hogar, reduciéndose así la brecha de participación por género.

Demografía. Históricamente, en muchas sociedades, la mujer ha tenido tenido un rol tradicional en el cuidado de la casa y de la familia lo que puede influir tanto en la decisión de participación como en la del número de horas trabajadas. En principio, se esperaría por ejemplo que mujeres casadas y con hijos tengan una menor participación promedio y una menor intensidad de oferta que mujeres solteras sin hijos, simplemente porque las primeras enfrentan un mayor costo de oportunidad de salir a trabajar. En otras palabras, existen factores demográficos (como la estructura del hogar y el tipo de hogar) que podrían potencialmente influir en la decisión de oferta laboral de las mujeres y no en la oferta laboral de los hombres.

Para analizar el efecto de la situación familiar en la existencia de discriminación se clasificó a los individuos en cuatro categorías familiares. En primer lugar, se define hogar 
conyugal a aquel que contiene una familia del tipo: (i) pareja sin hijos (jefe y cónyuge), (ii) pareja con uno o más hijos solteros, (iii) padre o madre con uno o más hijos solteros. De esta forma se dirá que un hogar es conyugal completo si ambos cónyuges están presentes en tanto que será incompleto si sólo un cónyuge está presente (hogar monoparental). En segundo lugar, se denomina parientes nucleares al jefe, cónyuge y a los hijos solteros del jefe; en tanto que los demás parientes (hijos casados, padres, suegros, nietos, etc.) se consideran parientes no nucleares. Finalmente, hay individuos que pertenecen a hogares no conyugales: son aquellos que no contienen un núcleo conyugal que incluya al jefe de hogar. Pueden estar formados por: un único individuo (hogar unipersonal), sólo parientes no nucleares, sólo no parientes, o por parientes no nucleares y no parientes.

Estas definiciones dan lugar a dos categorizaciones. Por un lado se clasifica a los hogares en: (1) completo nuclear, (2) completo no nuclear, (3) monoparental y (4) no conyugal. Asimismo, según el jefe sea hombre o mujer una segunda clasificación será: (1) hombrecompleto, (2) hombre-monoparental, (3) mujer-completo y (4) mujer-monoparental.

Como surge a simple vista en los Cuadros 6 a y 6b, las mayores brechas en casi todas las variables bajo análisis se verifican entre hombres y mujeres pertenecientes a grupos completos (nucleares y no nucleares), especialmente en aquellos cuyo jefe es hombre.

Nótese en primer término que las mujeres pertenecientes a familias completas tienden a tener una menor participación en el mercado laboral y a trabajar un menor número de horas por semana que el resto de las mujeres. A su vez, esto explicaría que las brechas por género de ingreso mensual sean más importantes en personas pertenecientes a estos grupos (mientras que la mujeres pertenecientes a hogares completos ganan un 68\%-69\% de lo que ganan los hombres de esos hogares, ese ratio aumenta al $84 \%$ para los no conyugales). Asimismo, es en los hogares completos donde se verifican también brechas en el ingreso horario favorables a los hombres, por contraposición a lo que ocurre en los no conyugales.

Sector Económico y Tipo de Empresa. Finalmente, un último aspecto que puede influir en la existencia de diferencias de ingreso y de formalidad laboral es el sector y el tipo de 
empresa en la que se trabaja. Específicamente, si las mujeres tienden a agruparse en sectores o empresas que pagan salarios bajos y contratan una gran proporción de trabajadores informales y los hombres lo hacen en empresas y sectores que pagan salarios altos y mantienen una alta proporción de su planta laboral de manera formal, entonces las brechas de ingreso y formalidad tenderán a ser mayores que en el caso en que hombres y mujeres estuvieran distribuidos de manera relativamente uniforme entre sectores y tipos de empresa. ${ }^{9}$

En el Cuadro 7 puede observarse que existen sectores donde predominan los hombres (como en el sector primario, en el sector construcción y en el sector transporte) y otros sectores donde hay una gran proporción de mujeres (como en los servicios domésticos y en el sector de provisión de servicios sociales). Asimismo, las mujeres tienden a trabajar más de manera unipersonal que los hombres.

Nótese, por ejemplo, que el salario horario promedio en el sector doméstico, sector predominantemente femenino, tiende por un lado a ser pequeño en relación al de otros sectores donde predominan los hombres (como el sector público) pero es mayor o igual al percibido en la construcción y en el sector primario, dos sectores donde la participación de los hombres es alta. La evidencia en materia de informalidad, es más clara. El sector de servicios domésticos y las empresas unipersonales presentan tasas de informalidad sustancialmente mayores que el resto de los sectores y tipos de empresas, lo que podría explicar en parte las mayores tasas de informalidad experimentadas por las mujeres.

Como hemos visto la educación, la experiencia, los factores demográficos y el tipo de firma donde trabajan hombres y mujeres parecen tener algún efecto en las diferencias entre hombres y mujeres en términos de ingreso mensual, salario horario, informalidad y desempleo. Naturalmente, el paso siguiente es realizar un análisis condicional de estas

\footnotetext{
9 Este fenómeno conocido como "segregación" puede ser una fuente adicional de discriminación. En particular, podría ocurrir que las mujeres, sabiendo que una vez que salgan al mercado laboral van a ser discriminadas, elijan trabajar en ciertos sectores o tipos de empresas donde la discriminación es menor. Existe también un patrón cultural, así como cuestiones de preferencias, que asignan distintas actividades a distintos sexos. Estos patrones generarán una brecha salarial que no será fruto de la discriminación sino en todo caso de diferencias compensatorias.
} 
diferencias intentando captar cuánto es explicado por diferencias en los retornos a estas características y cuánto es explicado por diferencias en las características mismas de hombres y mujeres.

\section{Medición de la discriminación: aspectos metodológicos}

Para detectar y cuantificar la existencia de discriminación salarial en el mercado laboral se utilizará la metodología presentada originalmente en Oaxaca (1973) que descompone la brecha salarial bruta en un componente de discriminación y otro de productividad. En Oaxaca y Ransom (1994) se presenta una estrategia que permite, a su vez, dividir al componente discriminatorio en dos: un primer término que indica cuánto se favorece a los hombres y un segundo componente que muestra cuánto se perjudica a las mujeres.

Por otra parte, para analizar la existencia de discriminación en materia de desempleo e informalidad se requerirá modificar la metodología anterior, basada en la estimación de modelos lineales, de forma tal de contemplar la estructura no lineal de los modelos de elección binaria utilizados para modelar la probabilidad de estar desempleado o de ser un trabajador informal. En este caso se seguirá la metodología desarrollada en Yun (2000).

\subsection{Discriminación a través de salarios}

Siguiendo a Oaxaca (1973), se define la brecha salarial bruta como $G_{m h}=\left(W_{h} / W_{m}\right)-1$. En ausencia de discriminación, y bajo el supuesto de mercados competitivos, esta brecha debería reflejar solamente diferencias en productividad. Esto es, $Q_{m h}=\left(W_{h}^{o} / W_{m}^{o}\right)-1=\left(P m g_{h} / P m g_{m}\right)-1$.

En este contexto, el coeficiente de discriminación $\left(D_{m h}\right)$ se define como la diferencia porcentual entre la brecha de salarios $\left(G_{m h}+1\right)$ y la brecha justificada por las diferencias en las características productivas de hombres y mujeres $\left(Q_{m h}+1\right)$. En símbolos, $D_{m h}=\left[\left(W_{h} / W_{m}\right)-\left(W_{h}^{o} / W_{m}^{o}\right)\right] /\left(W_{h}^{o} / W_{m}^{o}\right)$. De esta forma, se encuentra que, 


$$
\ln \left(G_{m h}+1\right)=\ln \left(D_{m h}+1\right)+\ln \left(Q_{m h}+1\right) .
$$

que indica que la brecha salarial bruta (o sea la observada) se puede descomponer en un término que indica cuánto de la misma se explica por discriminación y cuánto por diferencias en características productivas.

Oaxaca y Ransom (1994) proponen una descomposición adicional. En particular, el coeficiente de discriminación puede desagregarse en dos partes: una que indica cuánto beneficia el mercado a los hombres y otra que indica cuánto perjudica a las mujeres. Para ello, se define a la ventaja masculina como $\delta_{b o}=W_{b} / W_{b}^{o}-1$, que indica cuánto se remunera a los hombres por encima de su productividad marginal; y a la desventaja femenina como $\delta_{o m}=W_{m}^{\circ} / W_{m}-1$, que muestra cuánto se remunera a las mujeres por debajo de su productividad marginal.

Teniendo en cuenta estas definiciones y que $\ln \left(\mathrm{D}_{\mathrm{hm}}+1\right)=\ln \left[\left(\mathrm{W}_{\mathrm{h}} / \mathrm{W}_{\mathrm{m}}\right) /\left(\mathrm{W}_{\mathrm{h}}^{\circ} / \mathrm{W}_{\mathrm{m}}^{\circ}\right)\right]$, se puede escribir al coeficiente de discriminación como:

$$
\ln \left(\mathrm{D}_{\mathrm{hm}}+1\right)=\ln \left(\delta_{\mathrm{ho}}+1\right)+\ln \left(\delta_{\text {om }}+1\right)
$$

Finalmente, utilizando (1) y (2) tenemos que,

$$
\ln \left(\mathrm{G}_{\mathrm{hm}}+1\right)=\ln \left(\delta_{\mathrm{ho}}+1\right)+\ln \left(\delta_{\mathrm{om}}+1\right)+\ln \left(\mathrm{Q}_{\mathrm{mh}}+1\right)
$$

La forma estándar de estimar $D_{h m}$, consiste en hallar qué parte de la brecha salarial bruta no es explicada por las características productivas de los individuos. A tal efecto, la brecha salarial bruta promedio puede expresarse como $\ln \left(G_{m h}+1\right)=\ln \left(\tilde{\mathrm{W}}_{\mathrm{h}} / \tilde{\mathrm{W}}_{\mathrm{m}}\right)=\ln \left(\tilde{\mathrm{W}}_{\mathrm{h}}\right)-\ln \left(\tilde{\mathrm{W}}_{\mathrm{m}}\right)$, donde “ " indica media geométrica. 
La estrategia natural de estimación consiste entonces en estimar ecuaciones del logaritmo del salario separadas para hombres y mujeres y luego hacer uso de una propiedad básica del modelo lineal general; esto es que, $\ln \left(\tilde{W}_{h}\right)=\bar{X}_{h}^{\prime} \hat{\beta}_{h}$ y que $\ln \left(\tilde{W}_{m}\right)=\bar{X}_{m}^{\prime} \hat{\beta}_{m}$, donde “—“ “ indica promedio y “^” indica estimado. ${ }^{10}$

De esta forma, $\ln \left(G_{m h}+1\right)=\bar{X}_{h}^{\prime} \hat{\beta}_{h}-\bar{X}_{m}^{\prime} \hat{\beta}_{m}$. Si definimos a $\beta^{*}$ como el vector de coeficientes prevalecientes en la ecuación de salarios cuando no existe discriminación, sumando y restando $\overline{\mathrm{X}}_{\mathrm{h}}^{\prime} \beta^{*}$ y $\overline{\mathrm{X}}_{\mathrm{m}}^{\prime} \beta^{*}$ y rearreglando términos se obtiene que,

$$
\ln \left(G_{m h}+1\right)=\bar{X}_{h}^{\prime}\left(\hat{\beta}_{h}-\beta^{*}\right)+\bar{X}_{m}^{\prime}\left(\beta^{*}-\hat{\beta}_{m}\right)+\beta^{*}\left(\bar{X}_{h}^{\prime}-\bar{X}_{m}^{\prime}\right)
$$

La ecuación anterior muestra que la brecha bruta puede descomponerse en tres partes. El primer término corresponde al exceso del retorno recibido por los hombres multiplicado por sus características personales promedio. El segundo término indica la parte de la brecha explicada por el menor retorno recibido por las mujeres multiplicado por sus características personales promedio y, finalmente, el tercer término muestra la parte de la brecha bruta que es explicada por diferencias en características de hombres y mujeres, a los precios de factores prevalecientes en ausencia de discriminación. Por construcción, los dos primeros términos corresponden a $\ln \left(\delta_{\mathrm{mo}}+1\right)$ y a $\ln \left(\delta_{\mathrm{oh}}+1\right)$, respectivamente; en tanto que el tercer término corresponde a $\ln \left(\mathrm{Q}_{\mathrm{mh}}+1\right)$.

Oaxaca y Ransom (1988) y Neumark (1988) proponen que $\beta^{*}$ sea la estimación MCO de la muestra combinada de hombres y mujeres.

${ }^{10}$ Sea $\tilde{W}=\left(\prod_{i=1}^{N} w_{i}\right)^{\frac{1}{N}}$ la media geométrica de w. De modo que $\ln (\tilde{W})=\frac{\sum_{i=1}^{N} \ln \left(w_{i}\right)}{N}=\overline{\ln (w)}$, esto es la media aritmética del $\ln (\mathrm{w})$, la variable dependiente en una ecuación estándar del logaritmo del salario. Por 
Nótese además que la ecuación (4) permite conocer qué factores afectan a cada uno de los tres componentes mencionados. De hecho (4) puede reescribirse como,

$$
\ln \left(\mathrm{G}_{\mathrm{mh}}+1\right)=\sum_{\mathrm{i}=1}^{\mathrm{k}}\left[\overline{\mathrm{x}}_{\mathrm{hi}}^{\prime}\left(\hat{\beta}_{\mathrm{hi}}-\beta_{\mathrm{i}}^{*}\right)+\overline{\mathrm{x}}_{\mathrm{mi}}^{\prime}\left(\beta_{\mathrm{i}}^{*}-\hat{\beta}_{\mathrm{mi}}\right)+\beta_{\mathrm{i}}^{*}\left(\overline{\mathrm{x}}_{\mathrm{hi}}^{\prime}-\overline{\mathrm{x}}_{\mathrm{mi}}^{\prime}\right)\right]
$$

donde $k$ es el número de variables independientes en la ecuación de salarios. De forma tal que esta especificación permite identificar cuánto de la discriminación viene explicado, por ejemplo, por el hecho de que el mercado remunera más la características educativas de los hombres que las de las mujeres.

Por lo tanto, una gran parte de la discusión de cómo medir discriminación salarial reside en el método de estimación de la ecuación de salarios. ${ }^{11}$ Existen, en principio dos cuestiones adicionales a tener en cuenta.

En primer lugar, debemos definir qué variables explican el salario de los individuos. Siguiendo a Oaxaca (1973) en este trabajo se estimarán dos tipos de ecuaciones.

- Ecuaciones de características personales. En este caso la ecuación de salarios se especifica de manera estándar. Básicamente, a partir del modelo de Mincer (1974) se explica el logaritmo del salario a través de las características educativas y de experiencia laboral de los individuos.

- Ecuaciones completas. Alternativamente, puede alegarse que la percepción de discriminación en general ocurre cuando se remunera distinto a un hombre y a una mujer de características iguales y que desarrollan una misma tarea. En consecuencia se estimarán un segundo grupo de ecuaciones, que incorporan a las ecuaciones de

propiedad del estimador MCO, se tiene que $\overline{\ln (w)}=\bar{X} \hat{\beta}=\ln (\tilde{W})$.

${ }^{11}$ Kunze (2000) presenta un extensivo survey de esta cuestión. 
características personales, variables que indican el sector y el tamaño de la empresa en la que trabajan las personas. ${ }^{12}$

El segundo aspecto a tener en cuenta es la posible existencia de un sesgo de selección. Desde un punto de vista teórico sólo ingresarían al mercado laboral aquellos individuos cuya utilidad neta de trabajar sea positiva. Consecuentemente, sólo para estos individuos se observaría un salario positivo (para el resto se observará un salario igual a cero). En el caso particular de las mujeres existe un conjunto de factores observables e inobservables que hacen que tengan una participación menor que la de los hombres. Estos factores suelen estar correlacionados con la educación y con la experiencia de manera que no incorporarlos traería como consecuencia un sesgo en el vector de coeficientes estimados. Por lo tanto, se controla por la posible existencia de un sesgo de selección utilizando el procedimiento en dos etapas desarrollado en Heckman (1979). ${ }^{13}$

En suma, las ecuaciones de salario estimadas serán (omitiendo, para simplificar, los subíndices de individuos), ${ }^{14}$

$$
\begin{aligned}
\ln (\mathrm{w})=\beta_{0}+ & \sum_{\mathrm{i}=1}^{5} \beta_{\mathrm{i}} \mathrm{Edu}_{\mathrm{i}}+\beta_{6} \mathrm{E}+\beta_{7} \mathrm{E}^{2}+\beta_{8} \mathrm{~A}+\sum_{\mathrm{i}=9}^{11} \beta_{\mathrm{i}} \text { Tipo }_{\mathrm{i}}+ \\
& \sum_{\mathrm{i}=12}^{14} \beta_{\mathrm{i}} \text { Región }_{\mathrm{i}}+\beta_{15} \lambda+\delta\left[\sum_{\mathrm{i}=16}^{23} \beta_{\mathrm{i}} \text { Sector }_{\mathrm{i}}+\sum_{\mathrm{i}=24}^{29} \beta_{\mathrm{i}} \text { Tamaño }_{\mathrm{i}}\right]+\mathrm{e} ; \delta=\{0,1\}
\end{aligned}
$$

Donde, Edu son las categorías educativas; $E$ es la experiencia potencial; $A$ la antigüedad en el empleo actual; Tipo el tipo de trabajador (calificado, no calificado, profesional u otros); Región la región de residencia y $\lambda$ es una variable que controla por el sesgo de selección. ${ }^{15}$

\footnotetext{
${ }^{12}$ Nótese que, sin embargo, la incorporación de estas variables podría generar estimaciones inconsistentes si, por ejemplo, sabiendo que en el futuro van a ser discriminadas, las mujeres tendieran a educarse menos y por lo tanto esto limitara el acceso a determinados sectores o a determinados tipos de empresas.

${ }^{13}$ Se dejan de lado dos cuestiones que pueden resultar interesante analizar en investigaciones futuras: el conocido problema de endogeneidad en la ecuación de salarios y la posible existencia de diferencias en discriminación a lo largo de la distribución del ingreso.

${ }^{14}$ En el apéndice 1 se presenta detalladamente la definición y construcción de variables.

${ }^{15}$ En particular, $\lambda$ es la inversa del ratio de Mill asociado a la probabilidad de estar en la muestra
} 
$\delta$ toma valor cero en la ecuación de características personales y 1 en la ecuación completa. Sector hace referencia al sector donde está empleado el trabajador; Tamaño mide al tamaño de la firma en que trabaja y $e$ es el error aleatorio que se supone cumple con los supuestos clásicos. La variable dependiente $w$ se definió alternativamente como el ingreso mensual y como el salario horario. ${ }^{16}$

Asimismo, la ecuación de selección se estima a partir de un modelo probit que fue especificado de la siguiente manera:

$$
p=\mathrm{F}\left(\alpha_{0}+\sum_{\mathrm{i}=1}^{5} \alpha_{\mathrm{i}} \mathrm{Edu}_{\mathrm{i}}+\alpha_{6} \text { casado }+\alpha_{7} \text { hijosol }+\alpha_{8} \text { escuela }\right)
$$

donde $p$ indica la probabilidad de que el individuo participe en el mercado; casado es una variable dicotómica que indica si el individuo está casado; hijosol indica si la persona tiene a su cargo hijos solteros, y escuela si está asistiendo a la escuela.

\subsection{Discriminación a través del desempleo y la informalidad laboral}

La metodología de Oaxaca utilizada para medir discriminación en materia de ingresos y salarios debe ser ligeramente modificada para analizar el caso de la discriminación en desempleo e informalidad. Sin embargo, la estrategia de descomposición de la brecha salarial bruta se mantiene.

Supongamos que observamos la variable $\mathrm{S}_{\mathrm{gn}}$ que indica la situación de un individuo $n$ perteneciente al grupo g (hombres-mujeres). Básicamente, se observa si el trabajador es informal $\left(\mathrm{S}_{\mathrm{gn}}=1\right)$ o formal $\left(\mathrm{S}_{\mathrm{gn}}=0\right)$ o si está desempleado $\left(\mathrm{S}_{\mathrm{gn}}=1\right)$ o empleado $\left(\mathrm{S}_{\mathrm{gn}}=0\right)$. De esta forma, se estimará un modelo de elección binaria para la probabilidad de ser informal o ser desempleado,

$$
\mathrm{P}_{\mathrm{g}}=\Phi\left(\mathrm{Z}_{\mathrm{gn}} \gamma_{\mathrm{g}}\right)
$$

\footnotetext{
16 También se estimaron las ecuaciones de salario utilizando como variable dependiente el salario horario ajustado por trabajo parcial, siguiendo la metodología presentada en Paz (2000). Los resultados fueron
} 
Supongamos además que $\Phi($.$) es una distribución normal, resultando de esa forma un$ modelo probit. Nótese que, por construcción, $\overline{\mathrm{S}}_{\mathrm{g}}=\overline{\hat{\mathrm{P}}}=\overline{\Phi\left(\mathrm{Z}_{\mathrm{g}} \gamma_{\mathrm{g}}\right)}$. De manera que se puede definir la brecha bruta de probabilidades de ser informal o de ser desempleado, como:

$$
G_{h m}=\bar{S}_{h}-\bar{S}_{m}=\overline{\Phi\left(Z_{h} \gamma_{h}\right)}-\overline{\Phi\left(Z_{m} \gamma_{m}\right)}
$$

Esta brecha es análoga a la brecha bruta de ingresos analizada en la sección anterior. En este caso, la misma también se puede descomponer en un efecto características y un efecto discriminación. Básicamente, sumando y restando $\overline{\Phi\left(Z_{m} \gamma_{h}\right)}$ en (5) se obtiene,

$$
G_{b m}=Q_{b m}^{1}+D_{b m}^{1}=\left[\overline{\Phi\left(Z_{b} \gamma_{b}\right)}-\overline{\Phi\left(Z_{m} \gamma_{b}\right)}\right]+\left[\overline{\Phi\left(Z_{m} \gamma_{b}\right)}-\overline{\Phi\left(Z_{m} \gamma_{m}\right)}\right]
$$

Alternativamente se podría sumar y restar $\overline{\Phi\left(Z_{\mathrm{h}} \gamma_{\mathrm{m}}\right)}$ en (5) obteniendo,

$$
G_{h m}=Q_{h m}^{2}+D_{h m}^{2}=\left[\overline{\Phi\left(Z_{m} \gamma_{b}\right)}-\overline{\Phi\left(Z_{b} \gamma_{b}\right)}\right]+\left[\overline{\Phi\left(Z_{m} \gamma_{m}\right)}-\overline{\Phi\left(Z_{m} \gamma_{b}\right)}\right]
$$

Donde $\mathrm{Qhm}^{\mathrm{i}}$ y $\mathrm{D}_{\mathrm{hm}}{ }^{\mathrm{i}}$, representan el coeficiente de características y discriminación respectivamente.

Básicamente estas expresiones nos brindan una medida agregada del efecto diferencias en características individuales $\left(Q_{h m}{ }^{i}\right)$ y del efecto discriminación $\left(D_{h m}{ }^{i}\right)$. Sin embargo, dado que $\Phi($.$) es una función no lineal, no existe una única forma de encontrar el efecto de cada$ característica y de cada coeficiente por separado. De manera que podríamos obtener un cálculo agregado de la cuantía de la discriminación pero no sus determinantes.

Yun (2000) propone un método que emula los resultados obtenidos por Oaxaca (1973). Básicamente, allí se propone un método que consta de dos etapas. 
En una primer etapa se aproxima la media muestral de $\Phi($.$) con el valor de \Phi($.$) evaluado$ en la media de las características individuales. En símbolos, se sustituye $\overline{\Phi\left(Z_{\mathrm{g}} \gamma_{\mathrm{g}}\right)} \cong \Phi\left(\bar{Z}_{\mathrm{g}} \gamma_{\mathrm{g}}\right)$

La segunda etapa consiste en hallar una aproximación lineal alrededor de $\bar{Z}_{\mathrm{g}} \gamma_{\mathrm{g}^{\prime}}=\overline{\mathrm{Z}}_{\mathrm{g}} \gamma_{\mathrm{g}}$. En suma,

$$
\begin{aligned}
& \mathrm{G}_{\mathrm{hm}}^{1}=\left[\left(\bar{Z}_{\mathrm{h}}-\mathrm{Z}_{\mathrm{m}}\right) \gamma_{\mathrm{h}} \phi\left(\overline{\mathrm{Z}}_{\mathrm{h}} \gamma_{\mathrm{h}}\right)\right]+\left[\overline{\mathrm{Z}}_{\mathrm{m}}\left(\gamma_{\mathrm{h}}-\gamma_{\mathrm{m}}\right) \phi\left(\overline{\mathrm{Z}}_{\mathrm{m}} \gamma_{\mathrm{m}}\right)\right]+\mathrm{R}^{1} \\
& G_{h m}^{2}=\left[\left(\bar{Z}_{h}-Z_{m}\right) \gamma_{m} \phi\left(\bar{Z}_{m} \gamma_{m}\right)\right]+\left[\bar{Z}_{h}\left(\gamma_{h}-\gamma_{m}\right) \phi\left(\bar{Z}_{h} \gamma_{h}\right)\right]+R^{2}
\end{aligned}
$$

Donde $\phi($.$) es la densidad normal y los \mathrm{R}^{\mathrm{i}}$ son residuos que conforman la brecha y no están explicado en el resto de los términos. ${ }^{17}$

Sólo queda analizar cómo se estimaron los coeficientes $\gamma$ de cada uno de los modelos. Como en el caso de las ecuaciones de salario se estimaron dos tipos de ecuaciones (completas y de características personales ${ }^{18}$ ) Siguiendo un modelo teórico desarrollado en Gasparini (2000) se modeló la probabilidad de ser un trabajador informal como,

$$
\begin{aligned}
\mathrm{I}= & \Phi\left[\gamma_{0}+\sum_{\mathrm{i}=}^{5} \gamma_{\mathrm{i}} \mathrm{Edu}_{\mathrm{i}}+\gamma_{6} \mathrm{E}+\gamma_{7} \mathrm{E}^{2}+\gamma_{8} \mathrm{~A}+\sum_{\mathrm{i}=9}^{11} \gamma_{\mathrm{i}} \text { Tipo }_{\mathrm{i}}+\sum_{\mathrm{i}=12}^{14} \gamma_{\mathrm{i}} \text { Región }_{\mathrm{i}}+\right. \\
& \left.\delta\left[\sum_{\mathrm{i}=15}^{22} \gamma_{\mathrm{i}} \text { Sector }_{\mathrm{i}}+\sum_{\mathrm{i}=23}^{28} \gamma_{\mathrm{i}} \text { Tamaño }_{\mathrm{i}}\right]+\gamma_{29} \text { casado }+\gamma_{30} \text { hijosol }+\gamma_{31} \text { escuela }\right] ; \text { con } \delta=\{0,1\}
\end{aligned}
$$

En tanto que para la probabilidad de estar desempleado se estimó un modelo ad-hoc como el siguiente,

\footnotetext{
${ }^{17}$ Yun (2000) presenta un desarrollo más detallado de la metodología así como también una extensión al análisis de ecuaciones simultáneas.

${ }^{18}$ Para el caso de desempleo se utilizó el sector y el tipo de empresa en el cual realizó su último trabajo.
} 


$$
\begin{aligned}
\mathrm{D}= & \Phi\left[\gamma_{0}+\sum_{\mathrm{i}=1}^{5} \gamma_{\mathrm{i}} \text { Edu }_{\mathrm{i}}+\gamma_{6} \mathrm{E}+\gamma_{7} \mathrm{E}^{2}+\sum_{\mathrm{i}=9}^{11} \gamma_{\mathrm{i}} \text { Tipo }_{\mathrm{i}}+\sum_{\mathrm{i}=12}^{14} \gamma_{\mathrm{i}} \text { Región }_{\mathrm{i}}+\right. \\
& \left.\delta\left[\sum_{\mathrm{i}=15}^{22} \gamma_{\mathrm{i}} \text { Sector }_{\mathrm{i}}+\sum_{\mathrm{i}=23}^{28} \gamma_{\mathrm{i}} \text { Tamaño }_{\mathrm{i}}\right]+\gamma_{29} \text { casado }+\gamma_{30} \text { hijosol }+\gamma_{31} \text { escuela }\right] ; \text { con } \delta=\{0,1\}
\end{aligned}
$$

Por último, se hallaron intervalos de confianza para todas las medidas de discriminación utilizando para ello la técnica de bootstrap. La idea de esta técnica es obtener mediante una distribución de un estadístico de interés mediante simulaciones (que van modificando aleatoriamente la muestra utilizada). De esta forma, se puede hallar un error estándar asociado al estadístico calculado.

Nótese que en el caso del cálculo de las distribuciones de los distintos coeficientes de discriminación esto implica una complejidad computacional importante: por ejemplo, para calcular la discriminación en el salario horario (controlando por sesgo de selección) se deben estimar seis ecuaciones (tres con veintinueve parámetros y tres con nueve parámetros) y luego hacer los cálculos algebraicos explicados para obtener los coeficientes de interés. Por lo tanto, se calcularon los errores estándar en base a 500 repeticiones ( de todas maneras se probó que, en la muestras utilizadas y con los modelos estimados, a partir de las 200 repeticiones los resultados no cambian).

\section{Resultados empíricos}

El análisis empírico se divide en dos partes. En primer lugar, se presenta un resumen de los resultados hallados para GBA que permitirá obtener una idea acabada de la evolución a lo largo de los últimos quince años de la discriminación laboral, utilizando tal intervalos de confianza calculados por bootstrapping.

Luego se analiza la estructura de la discriminación laboral en Argentina en el periodo 19942000 buscando determinar si la discriminación se produjo a partir de un cierto favoritismo hacia los hombres o, en cambio, a través de un perjuicio contra las mujeres. Finalmente, en esa sección se hará hincapié en los determinantes observables de la discriminación laboral. 


\subsection{La evolución de la discriminación en GBA}

Existe cierta evidencia de que la discriminación laboral en Argentina parece estar decreciendo en el tiempo. En efecto, en las secciones anteriores se encontró cierta evidencia que muestra que la brecha salarial parece decrecer en el tiempo. ${ }^{19}$

Una primer pregunta que es interesante abordar entonces es si esa reducción de la brecha del salario horario está acompañada de una reducción en la discriminación. Asimismo, es interesante conocer qué ocurre mientras tanto con el resto de las variables del mercado laboral (ingreso mensual, informalidad y desempleo).

Para realizar el cálculo de la evolución de la discriminación salarial se utilizó las ecuaciones de características personales (y no las ecuaciones completas) presentadas en la sección anterior. En el Gráfico 3 puede observarse a simple vista que cuando se mide la discriminación salarial a través del ingreso mensual se encuentra que la misma es positiva persistentemente a lo largo de los últimos 15 años (algo que puede ser confirmado en el Cuadro 8 donde se presentan los estadísticos de significatividad individual). En promedio durante el periodo bajo análisis la discriminación resultó ser de alrededor del $42 \%$. Aunque esta es una medida difundida en los estudios de discriminación (Cain ,1986) tiene como desventaja que puede estar distorsionada por las preferencias de los individuos. Parte de la brecha no explicada se puede deber a factores no observables que hagan que las mujeres trabajen un menor número de horas al mes que los hombres y perciban por ende un ingreso mensual menor aún cuando tengan características productivas similares.

Por ello, puede considerarse que una medida más segura es la discriminación salarial calculada a partir del salario horario. En el Gráfico 3 se observa claramente que existe una tendencia decreciente en la discriminación salarial medida por el salario horario. El Cuadro 8 confirma esto: a partir de 1993 la discriminación salarial no es significativamente distinta de cero en el Gran Buenos Aires. ${ }^{20}$ Este resultado debe ser tomado como una señal positiva

\footnotetext{
${ }^{19}$ Los resultados de la estimación de los modelos para GBA no se presentan por cuestiones de espacio.

${ }^{20}$ Por otra parte, existe un "salto" en 1989 en ambas variables que podría ser fruto de problemas de medición debido a la alta inflación experimentada en ese período.
} 
pues estaría indicando la equiparación en el trato recibido por hombres y mujeres (al menos en el promedio).

Sin embargo, no todas han sido buenas noticias para las mujeres en materia de discriminación (Gráfico 4). En particular, se halló evidencia de que la reducción de discriminación por salarios se dio en un contexto de discriminación constante y positiva en materia de informalidad (Cuadro 9) y de discriminación creciente en términos de desempleo (Cuadro 10).

Por un lado, a partir de 1993 se produce un fuerte aumento de la discriminación por razón de desempleo. Nótese, sin embargo, que este fenómeno se da en un contexto de fuerte aumento del desempleo de hombres y mujeres. Por una parte, puede haber ocurrido que a partir de 1993 ingresaran al mercado laboral un gran número de mujeres con características productivas similares a las de los hombres. Muchos de los cuales ya estaban empleados en esa fecha y continuaron estándolo durante el periodo. Las condiciones económicas del momento impidieron a muchas de esas nuevas mujeres encontrar un empleo. Por otra parte, esta entrada de las mujeres al mercado puede haber puesto en evidencia un fenómeno de discriminación que antes no se observaba (por el menor número de mujeres en el mercado). Dados estos argumentos contrapuestos es importante ser cauteloso al momento de interpretar este aumento en la discriminación por desempleo.

En segundo lugar, se observa que la discriminación en materia de informalidad laboral ha permanecido en niveles positivos pero constantes (o levemente crecientes) en el tiempo. De hecho el coeficiente de discriminación por informalidad ha resultado significativo en todos los periodos.

El hecho de que esté tendiendo a desaparecer la discriminación por salario en tanto que continua existiendo algún tipo de discriminación por informalidad y desempleo es importante pues modifica completamente las prescripciones de política que se recomendarían en el caso argentino: dejan de ser importantes las políticas antidiscriminatorias del tipo "igual pago por igual trabajo" (presentes en la Constitución 
Nacional Argentina) y pasan a ser relevantes las políticas laborales que faciliten la contratación de trabajadores en igualdad de oportunidades.

\subsection{Estructura y determinantes de la discriminación laboral en Argentina}

Ingreso Mensual y Salario Horario. En los Cuadros 11 y 12 se presentan las estimaciones de las ecuaciones del logaritmo del salario horario y del ingreso mensual para el caso argentino, especificadas bajo la forma de características personales y bajo la forma completa para los años 1994 y $2000 .^{21}$

Los resultados son los esperados. Nótese que, en general, los coeficientes de las ecuaciones de los hombres tienden a ser mayores que los estimados para la muestra completa y éstos, a su vez, son superiores a los estimados para las mujeres. Esto nos da un indicio preliminar de la existencia de favoritismo hacia los hombres y perjuicio en contra de las mujeres. Estos hechos tienden a verificarse para ambas especificaciones y en los dos años bajo estudio. Además son más importantes para la ecuación de ingreso mensual y se verifican especialmente, para las dummies educativas, la experiencia potencial, el tipo de trabajador y el sector productivo. ${ }^{22}$

El Cuadro 13 resume los resultados hallados que surgen de aplicar la metodología de Oaxaca-Ransom al caso argentino. La brecha bruta en el ingreso mensual fue en 1994 del $37.1 \%$ y descendió en 2000 al 34.4\%. Las columnas (a) y (b) muestran la ventaja masculina y la desventaja femenina. Tanto en 1994 como en 2000 los hombres fueron favorecidos y las mujeres perjudicadas. Sin embargo, tanto el favoritismo como el perjuicio han tendido a reducirse en el tiempo implicando, naturalmente, una reducción de la discriminación. Asimismo, es importante notar que estos hechos son robustos ante cambios en la especificación de la ecuación de salarios.

\footnotetext{
${ }^{21}$ Las ecuaciones de selección presentaron los resultados estándar y pueden ser solicitadas al autor.

${ }^{22}$ En el caso de la comparación entre dummies debe notarse que la constante del modelo es mayor (o prácticamente igual ) en el caso de los hombres que en el de las mujeres -excepto en muy pocos casos.
} 
Por otro lado, la brecha bruta del salario horario fue negativa en ambos periodos. Como adelantamos en secciones anteriores, eso puede ser consistente con la existencia de discriminación si se diese en un contexto en el que las mujeres presentan características productivas superiores. Nótese que éste es el caso que se verifica en la Argentina. De hecho, los valores y la tendencia de la discriminación a favor de los hombres y en contra de las mujeres tienden a ser bastante parecidos a lo analizado para el caso del ingreso mensual.

Una cuestión que resulta relevante analizar es cuáles fueron los determinantes que jugaron a favor de los hombres y cuáles lo hicieron a favor de las mujeres. Para analizar esta cuestión se sumaron todos los factores que constituyeron una ventaja para los hombres y luego se sumaron los factores que se constituyeron como una ventaja a favor de las mujeres. Esto constituye lo que se denomina estructura vertical de la discriminación. ${ }^{23}$

En el Cuadro 14 se presenta la estructura vertical de la discriminación salarial para ambas especificaciones de la ecuación de salarios, tanto para el año 1994 como para el 2000.

En todos los casos, las variables asociadas al capital humano (educación, experiencia potencial y calificación) fueron las que más explicaron la discriminación en contra de las mujeres. Más importante aún, fue justamente la caída en estos coeficientes de discriminación los que básicamente explicaron la reducción de la discriminación salarial acaecida entre 1994 y 2000.

La antigüedad en el empleo y el tamaño de la firma tendieron a amortiguar el efecto discriminación, en tanto que la pertenencia a diferentes regiones no ha jugado ningún tipo de papel en la determinación del tamaño total del coeficiente de discriminación.

Informalidad y Desempleo. Asimismo, en los Cuadros 15 y 16 se muestran los resultados de estimar los modelos para la probabilidad de estar desempleado y para la probabilidad de

\footnotetext{
${ }^{23}$ Para la construcción de esta estructura y en pos de simplificar su exposición, se agruparon todos los coeficientes de discriminación correspondientes a cada grupo de variables bajo un único rótulo. Así, por ejemplo, los coeficientes correspondientes a las variables educativas se agruparon bajo el rótulo de Educación.
} 
ser informal. Puede observarse a simple vista que los signos de los coeficientes son los esperados: a mayor educación y mayor experiencia menor probabilidad de ser desempleado y de ser informal. Asimismo, las personas casadas tienen una menor probabilidad de estar desempleadas. En casi todos los casos los coeficientes negativos (o sea que corresponden a variables que reducen la probabilidad de ser desempleado o informal) tienden a ser mayores en valor absoluto para hombres que para mujeres; en tanto que los coeficientes con signos positivos tienden a ser mayores en valor absoluto para las mujeres que para los hombres. Esto indica que una vez más, a simple vista se observan retornos diferenciales a la educación, a la experiencia, y a las características demográficas.

En el Cuadro 17 se presenta un resumen de los resultados que surgen al aplicar la metodología de Yun (2000). Consistentemente con lo hallado para el caso del GBA, la brecha bruta de informalidad laboral entre mujeres y hombres en Argentina creció del 1.9\% en 1994 al 6\% en 2000. Además, este aumento en la brecha estuvo acompañado de un incremento en la discriminación. En 1994, la discriminación en términos de informalidad laboral era prácticamente nula (estimada entre el $-0.7 \%$ y el $0.9 \%$ mirando la ecuación completa y alrededor del $1.1 \%$ si se analiza la ecuación de características personales). En 2000, por su parte, las estimaciones de discriminación pasaron a ser del orden del 2.7\% / $2.9 \%$ en un caso y del $3.0 \% / 6.4 \%$ en otro.

Finalmente, en el caso de la probabilidad del desempleo, la brecha entre hombres y mujeres presentó una ligera caída, algo que como puede observarse también ocurrió en GBA. Llamativamente, al analizar el primer tipo de especificación (ecuación completa) se encuentra que la discriminación en términos de desempleo creció durante el período.

En el Cuadro 18 se presenta la estructura vertical de la discriminación a través de informalidad y desempleo. En el primer caso, tanto los factores asociados a la educación y a la experiencia potencial así como también los factores demográficos tendieron a aumentar la discriminación; en tanto que la antigüedad, el tipo de trabajador y el tamaño de la firma jugaron a favor de las mujeres reduciendo el tamaño de la discriminación total. En el segundo caso, sólo los factores demográficos tendieron a aumentar la discriminación. 
En suma, los resultados encontrados para Argentina indican que aún cuando la brecha bruta de salarios ha presentado una tendencia decreciente, aún persiste cierto tipo de discriminación salarial en contra de las mujeres. Este tipo de discriminación parece decrecer en el tiempo y esto está explicado tanto por una reducción del favoritismo hacia los hombres como por una disminución del perjuicio en contra de las mujeres.

Sin embargo, consistentemente con lo hallado para GBA, la reducción de la discriminación a través de salarios se produjo concomitantemente con un aumento de la discriminación en términos de la formalidad laboral y de la probabilidad de estar desempleado.

En términos simples, podría ocurrir que un hombre y una mujer de características iguales perciban un salario de bolsillo que tiende a igualarse a lo largo del tiempo pero que, sin embargo, las empresas estén aumentando la tendencia a contratar informalmente a las mujeres y manteniendo (o reduciendo) la proporción de hombres que contratan de manera informal. Algo similar, parece ocurrir en términos de a probabilidad de obtener un empleo.

Debe notarse finalmente que estos resultados son robustos ante cambios en la especificación del modelo y ante cambios en la muestra utilizada.

\section{Comentarios Finales}

En este trabajo se analizó la discriminación desde un punto de vista más amplio que el utilizado tradicionalmente. En general, los análisis de discriminación en el mercado laboral tienden a concentrarse en lo que ocurre en términos de los salarios y dejan de lado otros aspectos que pueden también ser fuente de discriminación (y no traducirse en una brecha salarial más amplia) como son la informalidad y el desempleo. En particular, hemos 
encontrado que aún cuando la brecha salarial ha tendido a cerrarse en los últimos años, persiste todavía un nivel de discriminación importante. Está brecha está básicamente explicada por diferencias en el trato que el mercado da al capital humano de hombres y mujeres. Asimismo, esta reducción en la discriminación salarial ocurrió concomitantemente a un aumento en la discriminación en la formalidad laboral y en la probabilidad del desempleo.

Este fenómeno de cambio en la forma de discriminación laboral implica la necesidad de un cambio en las políticas antidiscriminatorias. Básicamente además de la necesidad de implementar políticas tradicionales de "igual paga por igual trabajo" se requerirían políticas que igualen el tratamiento de hombres y mujeres en materia de formalidad y costo de contratación y despido. Naturalmente, una desregulación del mercado laboral y una mejora en los mecanismos de auditoria y control de obras sociales, seguros de trabajo y aportes a la seguridad social podrían lograr esta igualdad en el tratamiento de hombres y mujeres.

El análisis realizado sufre, sin embargo, de ciertas limitaciones. En primer lugar, se requeriría explorar mecanismos que controlen por la existencia del típico problema de endogeneidad que se encuentra en las ecuaciones de salarios y que puede conducir a estimaciones inconsistentes de los coeficientes y por ende del tamaño de la discriminación. La solución de este problema reside en la búsqueda de instrumentos que permitan eliminar dicha endogeneidad. Típicamente, las bases de datos utilizadas para este tipos de estudios carecen de información que sea utilizable como instrumentos. En segundo lugar, el análisis presentado estimó la discriminación promedio, dejando de lado la posible existencia de diferencias en el tamaño de la discriminación a lo largo de, por ejemplo, la distribución del ingreso. 


\section{Referencias}

Altonji, J. (1999). Race and Gender in the Labor Market. Handbook of Labor Economics, vol. 3, Chapter 48, Elsevier Ed.

Cain, S. (1986). The Economic Analysis of Labor Market. Handbook of Labor Economics, vol. 1, Chapter 13, Elsevier Ed.

Contreras, D. y M. Galván (2001). ¿Ha disminuido la discriminación salarial por género y etnia en bolivia ? Evidencia del período 1994 - 1999. Mimeo.

Ehrenberg, R., y R. Smith (2000) Modern Labor Economics: Theory and Public Policy. Seventh Edition, Addison Wesley.

Galiani, S. (2000). The Differential Evolution of Wages, Job Stability and Unemployment in Argentina: 1980-1998. Mimeo. Universidad de San Andrés.

Gasparini, L. (2000). Informalidad Laboral. En La Economía Oculta. FIEL.

Gasparini, L., M. Marchionni y W. Sosa Escudero (1999). A characterization of inequality changes in Argentina through microeconometric decompositions. Documento de Trabajo No 25. Departamento de Economía. Universidad Nacional de La Plata.

Hekman, J. (1979). Sample Selection Bias as a specification error. Econometrica. vol 47, nº 1.

Loureiro, P. (2001). Discriminacao, Salarios e densidades racial no Mercado de trabalho no Brasil. Texto para discussao. Serie Economa Ano 4, nº 25.

Oaxaca, R. (1973). Male-Female Wage Differential in Urban Labor Markets. International Economic Review. vol 14, no 3 .

Oaxaca, R. y M. Ransom (1994). On discrimination and the decomposition of wage differentials. Journal of Econometrics 61.

Paz, J. (1999) "La brecha entre géneros en Jujuy y Salta capital humano, segregación y selección muestral". Revista de la Escuela de Economía y Negocios, 1 (3) 137-154.

Yun, M. (2000). Decomposition Analisis for a Binary Choice Model. Institute for the Study of Labor (IZA). Discussion Paper $n^{\circ} 145$. 


\section{Cuadro 1}

Brechas laborales por género

Argentina, 1994 - 2000

\begin{tabular}{|c|c|c|c|c|c|c|}
\hline \multirow[b]{2}{*}{ Variable } & \multicolumn{3}{|c|}{1994} & \multicolumn{3}{|c|}{2000} \\
\hline & $\begin{array}{l}\text { Hombres } \\
\text { (a) }\end{array}$ & $\begin{array}{l}\text { Mujeres } \\
\text { (b) }\end{array}$ & $\begin{array}{c}\text { Brecha } \\
(\text { b) } /(\text { a)**100 }\end{array}$ & $\begin{array}{l}\text { Hombres } \\
\text { (a) }\end{array}$ & $\begin{array}{l}\text { Mujeres } \\
\text { (b) }\end{array}$ & $\begin{array}{l}\text { Brecha } \\
\text { (b) } /(a)^{\star} 100\end{array}$ \\
\hline Ingreso Laboral Horario (\$ corrientes) & 2.69 & 2.72 & 101 & 2.52 & 2.57 & 102 \\
\hline Ingreso Mensual (\$ corrientes) & 560 & 409 & 73 & 469 & 348 & 74 \\
\hline Informalidad (\%) & 36 & 34 & 95 & 29 & 35 & 122 \\
\hline Desempleo (\%) & 8 & 11 & 138 & 13 & 15 & 116 \\
\hline
\end{tabular}

Fuente: Elaboración propia en base a datos de la EPH.

Cuadro 2

Características promedio de la población argentina que participa del mercado laboral Argentina, 1994 - 2000

\begin{tabular}{|c|c|c|c|c|c|c|}
\hline & & 1994 & & & 2000 & \\
\hline Variable & $\begin{array}{l}\text { Hombres } \\
\text { (a) }\end{array}$ & $\begin{array}{l}\text { Mujeres } \\
\text { (b) }\end{array}$ & $\begin{array}{c}\text { Total } \\
\text { (c) }\end{array}$ & $\begin{array}{c}\text { Hombres } \\
\text { (a) }\end{array}$ & $\begin{array}{l}\text { Mujeres } \\
\text { (b) }\end{array}$ & $\begin{array}{c}\text { Total } \\
\text { (c) }\end{array}$ \\
\hline Primaria Incompleta & 0.11 & 0.09 & 0.10 & 0.09 & 0.07 & 0.08 \\
\hline Primaria Completa & 0.30 & 0.22 & 0.27 & 0.27 & 0.20 & 0.24 \\
\hline Secundaria Incompleta & 0.23 & 0.17 & 0.21 & 0.23 & 0.17 & 0.21 \\
\hline Secundaria Completa & 0.19 & 0.23 & 0.20 & 0.21 & 0.22 & 0.21 \\
\hline Superior Incompleta & 0.09 & 0.11 & 0.09 & 0.11 & 0.13 & 0.12 \\
\hline Superior Completa & 0.08 & 0.18 & 0.12 & 0.09 & 0.20 & 0.14 \\
\hline Experiencia Potencial & 21.01 & 19.23 & 20.32 & 20.92 & 19.64 & 20.39 \\
\hline Antigüedad & 4.35 & 4.19 & 4.29 & 4.27 & 4.13 & 4.21 \\
\hline Profesional & 0.08 & 0.08 & 0.08 & 0.07 & 0.07 & 0.07 \\
\hline Calificados & 0.65 & 0.42 & 0.56 & 0.68 & 0.48 & 0.60 \\
\hline No Calificado & 0.25 & 0.46 & 0.33 & 0.23 & 0.41 & 0.30 \\
\hline Otros & 0.01 & 0.03 & 0.02 & 0.02 & 0.03 & 0.02 \\
\hline Región 1 & 0.18 & 0.18 & 0.18 & 0.24 & 0.25 & 0.25 \\
\hline Región 2 & 0.21 & 0.21 & 0.21 & 0.15 & 0.16 & 0.16 \\
\hline Región 3 & 0.25 & 0.23 & 0.24 & 0.24 & 0.22 & 0.23 \\
\hline Región 4 & 0.36 & 0.37 & 0.37 & 0.37 & 0.36 & 0.37 \\
\hline Primario & 0.03 & 0.00 & 0.02 & 0.03 & 0.00 & 0.02 \\
\hline Industria & 0.17 & 0.08 & 0.14 & 0.14 & 0.07 & 0.11 \\
\hline Sector Público & 0.02 & 0.01 & 0.01 & 0.01 & 0.00 & 0.01 \\
\hline Construcción & 0.15 & 0.00 & 0.09 & 0.16 & 0.01 & 0.09 \\
\hline Comercio & 0.26 & 0.22 & 0.25 & 0.28 & 0.25 & 0.27 \\
\hline Transporte & 0.09 & 0.02 & 0.06 & 0.10 & 0.02 & 0.07 \\
\hline Servicios Sociales & 0.23 & 0.40 & 0.30 & 0.22 & 0.38 & 0.29 \\
\hline Servicio Doméstico & 0.02 & 0.22 & 0.10 & 0.02 & 0.21 & 0.10 \\
\hline Otros & 0.03 & 0.05 & 0.04 & 0.04 & 0.05 & 0.04 \\
\hline Tamaño 1 & 0.21 & 0.30 & 0.24 & 0.19 & 0.30 & 0.24 \\
\hline Tamaño 2 & 0.23 & 0.17 & 0.21 & 0.26 & 0.19 & 0.23 \\
\hline Tamaño 3 & 0.19 & 0.19 & 0.19 & 0.20 & 0.18 & 0.20 \\
\hline Tamaño 4 & 0.13 & 0.14 & 0.13 & 0.13 & 0.14 & 0.13 \\
\hline Tamaño 5 & 0.08 & 0.06 & 0.07 & 0.08 & 0.07 & 0.08 \\
\hline Tamaño 6 & 0.04 & 0.03 & 0.04 & 0.03 & 0.03 & 0.03 \\
\hline
\end{tabular}

Fuente: Elaboración propia en base a datos de la EPH. 
Cuadro 2 (Continuación)

Características promedio de la población argentina que participa del mercado laboral Argentina, 1994 - 2000

\begin{tabular}{lcccccc}
\hline \hline & \multicolumn{3}{c}{$\mathbf{1 9 9 4}$} & \multicolumn{3}{c}{$\mathbf{2 0 0 0}$} \\
\hline Variable & $\begin{array}{c}\text { Hombres } \\
(\mathrm{a})\end{array}$ & $\begin{array}{c}\text { Mujeres } \\
(\mathrm{b})\end{array}$ & $\begin{array}{c}\text { Total } \\
(\mathrm{c})\end{array}$ & $\begin{array}{c}\text { Hombres } \\
(\mathrm{a})\end{array}$ & $\begin{array}{c}\text { Mujeres } \\
(\mathrm{b})\end{array}$ & $\begin{array}{c}\text { Total } \\
(\mathrm{c})\end{array}$ \\
\hline Edad & 37 & 36 & 37 & 37 & 37 & 37 \\
Casado & 0.71 & 0.54 & 0.65 & 0.68 & 0.52 & 0.61 \\
Escuela & 0.04 & 0.07 & 0.05 & 0.06 & 0.09 & 0.07 \\
Hijos Solteros & 2.11 & 1.96 & 2.05 & 2.06 & 1.93 & 2.01 \\
\hline $\begin{array}{l}\text { Población que participa } \\
\text { del mercado laboral }\end{array}$ & 18178 & 11356 & 29534 & 14045 & 9762 & 23807 \\
$\begin{array}{l}\text { (observaciones) } \\
\text { Muestra: PEA 18-64 }\end{array}$ & 21405 & 23427 & 44832 & 16650 & 18297 & 34947 \\
$\begin{array}{l}\text { (observaciones) } \\
\text { Tasa de Participación }\end{array}$ & $\mathbf{0 . 8 5}$ & $\mathbf{0 . 4 8}$ & $\mathbf{0 . 6 6}$ & $\mathbf{0 . 8 4}$ & $\mathbf{0 . 5 3}$ & $\mathbf{0 . 6 8}$ \\
\hline
\end{tabular}

Fuente: Elaboración propia en base a datos de la EPH.

Cuadro 3

Evolución de la participación laboral, horas trabajadas y educación

Gran Buenos Aires , 1985-2000

\begin{tabular}{ccccccccccc}
\hline \hline & \multicolumn{3}{c}{$\begin{array}{c}\text { Participación } \\
\text { (\% de la PEA) }\end{array}$} & \multicolumn{3}{c}{$\begin{array}{c}\text { Horas Trabajadas } \\
\text { (Hs. Semanales) }\end{array}$} & \multicolumn{2}{c}{ Años de Educación } \\
\hline Año & Hombre & Mujer & Diferencia & Hombre & Mujer & Diferencia & Hombre & Mujer & Diferencia \\
\hline 1985 & 87.4 & 40.3 & 47.1 & 57 & 28 & 29.5 & 8.7 & 9.7 & -0.9 \\
1986 & 88.4 & 44.4 & 44.0 & 34 & 14 & 20.1 & 9.0 & 9.7 & -0.7 \\
1987 & 87.9 & 45.2 & 42.7 & 34 & 14 & 19.5 & 9.1 & 10.1 & -0.9 \\
1988 & 88.3 & 46.4 & 41.9 & 38 & 16 & 22.7 & 9.0 & 10.1 & -1.1 \\
1989 & 88.2 & 46.2 & 42.0 & 56 & 21 & 35.2 & 9.2 & 10.3 & -1.1 \\
1990 & 89.6 & 46.6 & 43.0 & 54 & 22 & 31.7 & 9.3 & 10.5 & -1.2 \\
1991 & 89.3 & 46.7 & 42.6 & 53 & 22 & 30.6 & 9.4 & 10.4 & -1.0 \\
1992 & 89.5 & 49.0 & 40.5 & 49 & 27 & 22.3 & 9.5 & 10.6 & -1.0 \\
1993 & 89.3 & 51.4 & 37.9 & 48 & 23 & 25.8 & 9.9 & 10.9 & -1.0 \\
1994 & 90.3 & 51.4 & 38.9 & 46 & 20 & 25.7 & 9.8 & 10.8 & -0.9 \\
1995 & 89.6 & 54.6 & 35.0 & 44 & 20 & 24.3 & 10.0 & 10.9 & -0.9 \\
1996 & 90.3 & 55.2 & 35.1 & 45 & 21 & 24.5 & 10.1 & 11.1 & -1.0 \\
1997 & 90.4 & 55.9 & 34.5 & 46 & 22 & 24.6 & 10.1 & 11.3 & -1.2 \\
1998 & 90.2 & 56.1 & 34.1 & 43 & 21 & 21.8 & 10.1 & 11.4 & -1.3 \\
1999 & 88.5 & 57.5 & 31.0 & 40 & 20 & 19.4 & 10.2 & 11.4 & -1.3 \\
2000 & 89.9 & 57.0 & 32.9 & 43 & 22 & 21.0 & 10.4 & 11.5 & -1.2 \\
Promedio & 89.2 & 50.2 & 39.0 & 45.7 & 20.8 & 24.9 & 9.6 & 10.7 & -1.0 \\
\hline
\end{tabular}

Fuente: Elaboración propia en base a datos de la EPH. 
Cuadro 4

Brechas en las principales variables laborales, por grupos etáreos

\begin{tabular}{l|ccc|ccc}
\multicolumn{5}{c}{ Argentina, $1994-2000$} \\
\hline
\end{tabular}

Fuente: Elaboración propia en base a datos de la EPH. Nota: \% indica la relación entre el valor observado para las mujeres y el observado para los hombres de una misma categoría.

\section{Cuadro 5}

Brechas en las principales variables laborales, por nivel de educación

Argentina, 1994-2000

\begin{tabular}{|c|c|c|c|c|c|c|}
\hline & \multicolumn{3}{|c|}{1994} & \multicolumn{3}{|c|}{2000} \\
\hline & Mujer & Hombre & $\%$ & Mujer & Hombre & $\%$ \\
\hline \multicolumn{7}{|c|}{ Ingreso Mensual (\$ Corr.) } \\
\hline Primario & 265 & 420 & 63 & 215 & 324 & 66 \\
\hline Secundario & 403 & 567 & 71 & 308 & 450 & 68 \\
\hline Superior & 561 & 863 & 65 & 503 & 713 & 70 \\
\hline \multicolumn{7}{|c|}{ Ingreso Horario (\$ Corr.) } \\
\hline Primario & 1.9 & 2.1 & 93 & 1.8 & 1.9 & 95 \\
\hline Secundario & 2.4 & 2.6 & 93 & 2.2 & 2.5 & 90 \\
\hline Superior & 4.3 & 5.1 & 84 & 3.9 & 4.3 & 90 \\
\hline \multicolumn{7}{|c|}{ Horas Trabajadas (Semanal) } \\
\hline Primario & 13 & 41 & 33 & 14 & 36 & 39 \\
\hline Secundario & 18 & 41 & 44 & 18 & 37 & 47 \\
\hline Superior & 21 & 39 & 54 & 22 & 36 & 61 \\
\hline \multicolumn{7}{|c|}{ Tasa de Participación (\%) } \\
\hline Primario & 38 & 87 & 44 & 45 & 89 & 51 \\
\hline Secundario & 50 & 86 & 58 & 53 & 85 & 62 \\
\hline Superior & 65 & 79 & 82 & 64 & 78 & 82 \\
\hline \multicolumn{7}{|c|}{ Tasa de Desempleo (\%) } \\
\hline Primario & 12 & 8 & 150 & 17 & 15 & 113 \\
\hline Secundario & 13 & 9 & 144 & 18 & 13 & 138 \\
\hline Superior & 9 & 6 & 150 & 10 & 8 & 125 \\
\hline \multicolumn{7}{|c|}{ Tasa de Informalidad (\%) } \\
\hline Primario & 39 & 24 & 163 & 57 & 37 & 154 \\
\hline Secundario & 19 & 20 & 95 & 37 & 29 & 128 \\
\hline Superior & 10 & 11 & 91 & 18 & 16 & 113 \\
\hline
\end{tabular}

Fuente: Elaboración propia en base a datos de la EPH. Nota: Idem Cuadro anterior 
Cuadro 6.a

Brechas en las principales variables laborales, por tipo y estructura de Familia Argentina, 1994-2000

\begin{tabular}{|c|c|c|c|c|c|c|}
\hline & \multicolumn{3}{|c|}{1994} & \multicolumn{3}{|c|}{2000} \\
\hline & Mujer & Hombre & $\%$ & Mujer & Hombre & $\%$ \\
\hline \multicolumn{7}{|c|}{ Ingreso Mensual (\$ Corr.) } \\
\hline Completo Nuclear & 407 & 590 & 69 & 354 & 503 & 70 \\
\hline Completo no Nuclear & 324 & 473 & 68 & 270 & 372 & 73 \\
\hline Monoparental & 380 & 376 & 101 & 317 & 305 & 104 \\
\hline No conyugal & 380 & 455 & 84 & 327 & 372 & 88 \\
\hline \multicolumn{7}{|c|}{ Ingreso Horario (\$ Corr.) } \\
\hline Completo Nuclear & 2.9 & 3.0 & 97 & 2.8 & 2.7 & 103 \\
\hline Completo no Nuclear & 2.2 & 2.3 & 98 & 2.1 & 2.1 & 98 \\
\hline Monoparental & 2.5 & 2.0 & 125 & 2.4 & 2.0 & 121 \\
\hline No conyugal & 3.2 & 2.4 & 132 & 2.5 & 2.5 & 101 \\
\hline \multicolumn{7}{|c|}{ Horas Trabajadas (Sem.) } \\
\hline Completo Nuclear & 15 & 43 & 35 & 16 & 40 & 41 \\
\hline Completo no Nuclear & 17 & 40 & 42 & 17 & 34 & 49 \\
\hline Monoparental & 25 & 34 & 73 & 21 & 30 & 71 \\
\hline No conyugal & 20 & 39 & 52 & 23 & 32 & 70 \\
\hline \multicolumn{7}{|c|}{ Tasa de Participación (\%) } \\
\hline Completo Nuclear & 44 & 87 & 51 & 49 & 87 & 56 \\
\hline Completo no Nuclear & 46 & 84 & 55 & 50 & 83 & 60 \\
\hline Monoparental & 66 & 79 & 84 & 67 & 78 & 86 \\
\hline No conyugal & 54 & 78 & 69 & 57 & 80 & 71 \\
\hline \multicolumn{7}{|c|}{ Tasa de Desempleo (\%) } \\
\hline Completo Nuclear & 11 & 7 & 157 & 13 & 10 & 130 \\
\hline Completo no Nuclear & 13 & 9 & 144 & 21 & 17 & 124 \\
\hline Monoparental & 12 & 14 & 86 & 16 & 20 & 80 \\
\hline No conyugal & 10 & 9 & 111 & 10 & 15 & 67 \\
\hline \multicolumn{7}{|c|}{ Tasa de Informalidad (\%) } \\
\hline Completo Nuclear & 19 & 17 & 112 & 30 & 25 & 120 \\
\hline Completo no Nuclear & 29 & 23 & 126 & 43 & 34 & 126 \\
\hline Monoparental & 24 & 32 & 75 & 38 & 42 & 90 \\
\hline No conyugal & 21 & 23 & 91 & 35 & 32 & 109 \\
\hline
\end{tabular}

Fuente: Elaboración propia en base a datos de la EPH. Nota: \% indica la relación entre el valor observado para las mujeres y el observado para los hombres de una misma categoría. 


\section{Cuadro 6.b}

Brechas en las principales variables laborales, por tipo y estructura de Familia

\begin{tabular}{|c|c|c|c|c|c|c|}
\hline & \multicolumn{3}{|c|}{1994} & \multicolumn{3}{|c|}{2000} \\
\hline & Mujer & Hombre & $\%$ & Mujer & Hombre & $\%$ \\
\hline \multicolumn{7}{|l|}{ Inqreso Mensual (\$ Corr.) } \\
\hline Hombre-completo & 384 & 567 & 68 & 334 & 478 & 70 \\
\hline Hombre-monoparental & 317 & 441 & 72 & 305 & 372 & 82 \\
\hline Mujer-completo & 392 & 416 & 94 & 308 & 324 & 95 \\
\hline Mujer-monoparental & 384 & 340 & 113 & 317 & 273 & 116 \\
\hline \multicolumn{7}{|l|}{ Ingreso Horario (\$ Corr.) } \\
\hline Hombre-completo & 2.7 & 2.8 & 97 & 2.6 & 2.6 & 101 \\
\hline Hombre-monoparental & 2.4 & 2.4 & 99 & 2.4 & 2.2 & 111 \\
\hline Mujer-completo & 2.2 & 2.3 & 95 & 2.4 & 2.0 & 120 \\
\hline $\begin{array}{l}\text { Mujer-monoparental } \\
\text { Horas Trabajadas (Sem.) }\end{array}$ & 2.5 & 1.8 & 138 & 2.4 & 1.8 & 128 \\
\hline Hombre-completo & 15 & 43 & 36 & 16 & 39 & 42 \\
\hline Hombre-monoparental & 15 & 34 & 45 & 15 & 36 & 43 \\
\hline Mujer-completo & 21 & 35 & 61 & 22 & 35 & 61 \\
\hline Mujer-monoparental & 26 & 34 & 76 & 22 & 27 & 80 \\
\hline \multicolumn{7}{|l|}{ Tasa de Participación (\%) } \\
\hline Hombre-completo & 44 & 86 & 51 & 49 & 86 & 57 \\
\hline Hombre-monoparental & 50 & 80 & 63 & 53 & 82 & 65 \\
\hline Mujer-completo & 56 & 83 & 67 & 64 & 84 & 76 \\
\hline Mujer-monoparental & 67 & 79 & 85 & 68 & 76 & 89 \\
\hline \multicolumn{7}{|l|}{ Tasa de Desempleo (\%) } \\
\hline Hombre-completo & 11 & 7 & 157 & 15 & 11 & 136 \\
\hline Hombre-monoparental & 15 & 10 & 150 & 18 & 12 & 150 \\
\hline Mujer-completo & 11 & 10 & 110 & 20 & 16 & 125 \\
\hline Mujer-monoparental & 12 & 16 & 75 & 16 & 24 & 67 \\
\hline \multicolumn{7}{|l|}{ Tasa de Informalidad (\%) } \\
\hline Hombre-completo & 21 & 18 & 117 & 33 & 26 & 127 \\
\hline Hombre-monoparental & 31 & 27 & 115 & 34 & 41 & 83 \\
\hline Mujer-completo & 34 & 23 & 148 & 36 & 36 & 100 \\
\hline Mujer-monoparental & 23 & 34 & 68 & 39 & 43 & 91 \\
\hline
\end{tabular}

Fuente: Elaboración propia en base a datos de la EPH. Nota: \% indica la relación entre el valor observado para las mujeres y el observado para los hombres de una misma categoría.

\section{Cuadro 7}

Análisis por sectores y tamaño de empresa

\begin{tabular}{|c|c|c|c|c|c|c|}
\hline & \multicolumn{2}{|c|}{$\begin{array}{c}\text { Participación } \\
\text { Masculina }\end{array}$} & \multicolumn{2}{|c|}{ Salario Horario } & \multicolumn{2}{|c|}{ Informalidad } \\
\hline & 1,994 & 2000 & 1,994 & 2000 & 1,994 & 2000 \\
\hline Primario & 92 & 91 & 1.8 & 2.9 & 22 & 29 \\
\hline Industria & 76 & 74 & 2.3 & 2.3 & 18 & 29 \\
\hline Sector Público & 84 & 87 & 4.2 & 4.0 & 4 & 11 \\
\hline Construcción & 98 & 98 & 2.0 & 1.9 & 46 & 53 \\
\hline Comercio & 65 & 61 & 2.2 & 2.0 & 28 & 38 \\
\hline Transporte & 89 & 86 & 2.7 & 2.3 & 30 & 41 \\
\hline Financiero & 66 & 61 & 5.2 & 4.7 & 6 & 11 \\
\hline Sector Social & 48 & 45 & 3.9 & 3.7 & 6 & 14 \\
\hline Servicios Domésticos & 13 & 13 & 2.2 & 1.9 & 61 & 77 \\
\hline 1 Persona & 53 & 49 & 2.3 & 2.0 & 58 & 75 \\
\hline 2-5 Personas & 67 & 66 & 2.1 & 2.1 & 39 & 55 \\
\hline 6-25 personas & 62 & 61 & 2.9 & 2.8 & 16 & 25 \\
\hline $26-100$ personas & 60 & 58 & 3.3 & 3.4 & 6 & 11 \\
\hline $101-500$ personas & 66 & 64 & 3.6 & 3.7 & 5 & 7 \\
\hline más de 500 personas & 70 & 64 & 4.0 & 4.2 & 4 & 7 \\
\hline
\end{tabular}

Fuente: Elaboración propia en base a datos de la EPH. 


\section{Cuadro 8}

Discriminación salarial

Gran Buenos Aires, 1985-2000

\begin{tabular}{|c|c|c|c|c|c|c|}
\hline \multirow[b]{2}{*}{ Año } & \multicolumn{3}{|c|}{ Log del Salario Horario } & \multicolumn{3}{|c|}{ Log del Ingreso Mensual } \\
\hline & Discriminación & $\begin{array}{c}\text { Desvío } \\
\text { Estándar }\end{array}$ & $\begin{array}{c}\text { Estadístico } \\
t\end{array}$ & Discriminación & $\begin{array}{l}\text { Desvío } \\
\text { Estándar }\end{array}$ & $\begin{array}{c}\text { Estadístico } \\
\mathrm{t}\end{array}$ \\
\hline 1985 & 0.263 & 0.053 & 4.993 & 0.476 & 0.061 & 7.775 \\
\hline 1986 & 0.261 & 0.056 & 4.648 & 0.471 & 0.069 & 6.833 \\
\hline 1987 & 0.128 & 0.048 & 2.686 & 0.456 & 0.064 & 7.185 \\
\hline 1988 & 0.285 & 0.066 & 4.320 & 0.561 & 0.083 & 6.727 \\
\hline 1989 & 0.431 & 0.080 & 5.383 & 0.765 & 0.106 & 7.222 \\
\hline 1990 & 0.126 & 0.084 & 1.488 & 0.357 & 0.110 & 3.244 \\
\hline 1991 & 0.057 & 0.068 & 0.851 & 0.257 & 0.076 & 3.368 \\
\hline 1992 & 0.120 & 0.057 & 2.117 & 0.281 & 0.068 & 4.136 \\
\hline 1993 & -0.028 & 0.090 & -0.312 & 0.368 & 0.076 & 4.827 \\
\hline 1994 & 0.044 & 0.096 & 0.462 & 0.483 & 0.065 & 7.461 \\
\hline 1995 & 0.077 & 0.091 & 0.854 & 0.456 & 0.079 & 5.749 \\
\hline 1996 & -0.043 & 0.057 & -0.758 & 0.294 & 0.069 & 4.249 \\
\hline 1997 & 0.125 & 0.067 & 1.862 & 0.323 & 0.076 & 4.265 \\
\hline 1998 & 0.132 & 0.054 & 2.469 & 0.518 & 0.077 & 6.762 \\
\hline 1999 & 0.096 & 0.054 & 1.759 & 0.342 & 0.065 & 5.296 \\
\hline 2000 & 0.059 & 0.046 & 1.300 & 0.404 & 0.072 & 5.595 \\
\hline
\end{tabular}

Fuente: Elaboración propia en base a datos de la EPH.

\section{Cuadro 9}

Discriminación en informalidad laboral Gran Buenos Aires, 1985-2000

\begin{tabular}{lcccccc}
\hline \hline Año & $\begin{array}{c}\text { Discriminación } \\
\text { I }\end{array}$ & $\begin{array}{c}\text { Desvío } \\
\text { Estándar }\end{array}$ & $\begin{array}{c}\text { Estadístico } \\
\mathbf{t}\end{array}$ & $\begin{array}{c}\text { Discriminación } \\
\mathbf{I}\end{array}$ & $\begin{array}{c}\text { Desvío } \\
\text { Estándar }\end{array}$ & $\begin{array}{c}\text { Estadístico } \\
\mathbf{t}\end{array}$ \\
\hline 1985 & 0.064 & 0.013 & 4.837 & 0.047 & 0.018 & 2.686 \\
1986 & 0.087 & 0.011 & 8.155 & 0.063 & 0.019 & 3.353 \\
1987 & 0.089 & 0.011 & 7.751 & 0.069 & 0.020 & 3.459 \\
1988 & 0.089 & 0.014 & 6.199 & 0.050 & 0.019 & 2.600 \\
1989 & 0.071 & 0.013 & 5.292 & 0.037 & 0.017 & 2.146 \\
1990 & 0.083 & 0.017 & 5.000 & 0.038 & 0.023 & 1.6337 \\
1991 & 0.082 & 0.023 & 3.593 & 0.028 & 0.024 & 1.1342 \\
1992 & 0.096 & 0.016 & 5.872 & 0.036 & 0.022 & 1.6688 \\
1993 & 0.096 & 0.020 & 4.845 & 0.059 & 0.024 & 2.4762 \\
1994 & 0.060 & 0.018 & 3.369 & 0.011 & 0.021 & 0.5089 \\
1995 & 0.091 & 0.017 & 5.441 & 0.042 & 0.022 & 1.9205 \\
1996 & 0.087 & 0.019 & 4.647 & 0.030 & 0.022 & 1.3557 \\
1997 & 0.109 & 0.019 & 5.640 & 0.060 & 0.023 & 2.5909 \\
1998 & 0.098 & 0.018 & 5.453 & 0.038 & 0.021 & 1.8222 \\
1999 & 0.104 & 0.017 & 5.950 & 0.065 & 0.021 & 3.0978 \\
2000 & 0.124 & 0.018 & 6.927 & 0.071 & 0.021 & 3.3304 \\
\hline
\end{tabular}

Fuente: Elaboración propia en base a datos de la EPH. 


\section{Cuadro 10}

Discriminación en desempleo

Gran Buenos Aires, 1985-2000

\begin{tabular}{lcccccc}
\hline \hline Año & $\begin{array}{c}\text { Discriminación } \\
\text { I }\end{array}$ & $\begin{array}{c}\text { Desvío } \\
\text { Estándar }\end{array}$ & $\begin{array}{c}\text { Estadístico } \\
\mathbf{t}\end{array}$ & $\begin{array}{c}\text { Discriminación } \\
\text { II }\end{array}$ & $\begin{array}{c}\text { Desvío } \\
\text { Estándar }\end{array}$ & $\begin{array}{c}\text { Estadístico } \\
\mathbf{t}\end{array}$ \\
\hline 1985 & -0.003 & 0.007 & -0.452 & -0.002 & 0.006 & -0.265 \\
1986 & 0.002 & 0.006 & 0.269 & 0.001 & 0.007 & 0.121 \\
1987 & 0.013 & 0.005 & 2.408 & 0.009 & 0.008 & 1.128 \\
1988 & 0.014 & 0.005 & 2.787 & 0.008 & 0.008 & 1.111 \\
1989 & -0.005 & 0.009 & -0.550 & -0.004 & 0.007 & -0.532 \\
1990 & -0.008 & 0.009 & -0.850 & -0.003 & 0.008 & -0.394 \\
1991 & 0.003 & 0.007 & 0.462 & 0.003 & 0.008 & 0.356 \\
1992 & 0.003 & 0.008 & 0.315 & 0.000 & 0.009 & -0.035 \\
1993 & 0.034 & 0.007 & 4.878 & 0.042 & 0.013 & 3.260 \\
1994 & 0.045 & 0.008 & 5.924 & 0.043 & 0.012 & 3.505 \\
1995 & 0.059 & 0.009 & 6.514 & 0.066 & 0.015 & 4.480 \\
1996 & 0.059 & 0.009 & 6.289 & 0.060 & 0.014 & 4.156 \\
1997 & 0.051 & 0.008 & 6.261 & 0.063 & 0.014 & 4.385 \\
1998 & 0.046 & 0.007 & 6.197 & 0.054 & 0.013 & 4.231 \\
1999 & 0.040 & 0.008 & 4.734 & 0.035 & 0.012 & 2.932 \\
2000 & 0.043 & 0.009 & 4.846 & 0.035 & 0.013 & 2.815 \\
\hline
\end{tabular}

Fuente: Elaboración propia en base a datos de la EPH. 


\section{Cuadro 11}

Ecuación del Log del Ingreso Mensual, MCO ajustado por sesgo de selección Argentina, 1994-2000

\begin{tabular}{|c|c|c|c|c|c|c|c|c|c|c|c|c|}
\hline & \multicolumn{6}{|c|}{ Ecuación Completa } & \multicolumn{6}{|c|}{ Ecuación de Características Personales } \\
\hline & \multicolumn{3}{|c|}{1994} & \multicolumn{3}{|c|}{2000} & \multicolumn{3}{|c|}{1994} & \multicolumn{3}{|c|}{2000} \\
\hline & Hombre & Mujer & Total & Hombre & Mujer & Total & Hombre & Mujer & Total & Hombre & Mujer & Total \\
\hline Constante & $\begin{array}{c}6.068 \\
(41.059)\end{array}$ & $\begin{array}{c}5.952 \\
(33.409)\end{array}$ & $\begin{array}{c}\mathbf{5 . 2 3 8} \\
(43.091)\end{array}$ & $\begin{array}{c}\mathbf{5 . 0 9 7} \\
(37.670)\end{array}$ & $\begin{array}{c}\mathbf{5 . 0 9 0} \\
(27.911)\end{array}$ & $\begin{array}{c}\mathbf{5 . 1 3 2} \\
(45.212)\end{array}$ & $\begin{array}{c}\mathbf{5 . 9 4 7} \\
(40.393)\end{array}$ & $\begin{array}{c}\mathbf{5 . 5 9 6} \\
(31.446)\end{array}$ & $\begin{array}{c}4.971 \\
(40.483)\end{array}$ & $\begin{array}{c}4.757 \\
(34.942)\end{array}$ & $\begin{array}{c}4.898 \\
(26.943)\end{array}$ & $\begin{array}{c}4.855 \\
(42.405)\end{array}$ \\
\hline Primaria Completa & $\begin{array}{c}\mathbf{0 . 1 8 3} \\
(10.804)\end{array}$ & $\begin{array}{c}\mathbf{0 . 1 2 1} \\
(4.814)\end{array}$ & $\begin{array}{c}\mathbf{0 . 1 7 2} \\
(11.851)\end{array}$ & $\begin{array}{c}\mathbf{0 . 1 4 2} \\
(5.868)\end{array}$ & $\begin{array}{c}0.103 \\
(2.926)\end{array}$ & $\begin{array}{c}\mathbf{0 . 1 2 5} \\
(6.026)\end{array}$ & $\begin{array}{c}\mathbf{0 . 2 1 2} \\
(12.346)\end{array}$ & $\begin{array}{c}\mathbf{0 . 1 7 7} \\
(6.883)\end{array}$ & $\begin{array}{c}\mathbf{0 . 2 1 4} \\
(14.299)\end{array}$ & $\begin{array}{c}\mathbf{0 . 1 7 9} \\
(7.106)\end{array}$ & $\begin{array}{c}\mathbf{0 . 1 1 6} \\
(3.175)\end{array}$ & $\begin{array}{c}\mathbf{0 . 1 4 8} \\
(6.853)\end{array}$ \\
\hline Secundaria Incompleta & $\begin{array}{c}\mathbf{0 . 3 4 9} \\
(18.729)\end{array}$ & $\begin{array}{c}\mathbf{0 . 2 3 9} \\
(8.342)\end{array}$ & $\begin{array}{c}\mathbf{0 . 3 2 8} \\
(20.021)\end{array}$ & $\begin{array}{c}\mathbf{0 . 3 2 9} \\
(12.806)\end{array}$ & $\begin{array}{c}\mathbf{0 . 1 6 7} \\
(4.287)\end{array}$ & $\begin{array}{c}\mathbf{0 . 2 5 8} \\
(11.524)\end{array}$ & $\begin{array}{c}\mathbf{0 . 3 9 6} \\
(21.171)\end{array}$ & $\begin{array}{c}\mathbf{0 . 3 3 9} \\
(11.717)\end{array}$ & $\begin{array}{c}\mathbf{0 . 3 9 2} \\
(23.510)\end{array}$ & $\begin{array}{c}0.389 \\
(14.634)\end{array}$ & $\begin{array}{c}\mathbf{0 . 1 9 8} \\
(4.939)\end{array}$ & $\begin{array}{c}\mathbf{0 . 2 9 9} \\
(12.927)\end{array}$ \\
\hline Secundaria Completa & $\begin{array}{c}\mathbf{0 . 5 7 6} \\
(29.587)\end{array}$ & $\begin{array}{c}\mathbf{0 . 4 1 4} \\
(14.038)\end{array}$ & $\begin{array}{c}\mathbf{0 . 5 0 2} \\
(29.926)\end{array}$ & $\begin{array}{c}\mathbf{0 . 5 4 4} \\
(20.180)\end{array}$ & $\begin{array}{c}\mathbf{0 . 3 9 9} \\
(9.563)\end{array}$ & $\begin{array}{c}\mathbf{0 . 4 5 4} \\
(19.087)\end{array}$ & $\begin{array}{c}\mathbf{0 . 6 3 2} \\
(32.529)\end{array}$ & $\begin{array}{c}\mathbf{0 . 5 7 1} \\
(19.732)\end{array}$ & $\begin{array}{c}0.575 \\
(34.142)\end{array}$ & $\begin{array}{c}\mathbf{0 . 6 3 5} \\
(23.001)\end{array}$ & $\begin{array}{c}0.472 \\
(11.141)\end{array}$ & $\begin{array}{c}\mathbf{0 . 5 1 8} \\
(21.281)\end{array}$ \\
\hline Superior Incompleta & $\begin{array}{c}0.738 \\
(31.298)\end{array}$ & $\begin{array}{c}0.493 \\
(14.037)\end{array}$ & $\begin{array}{c}\mathbf{0 . 6 1 9} \\
(30.851)\end{array}$ & $\begin{array}{c}0.710 \\
(23.466)\end{array}$ & $\begin{array}{c}\mathbf{0 . 5 0 8} \\
(11.380)\end{array}$ & $\begin{array}{c}\mathbf{0 . 5 7 6} \\
(22.431)\end{array}$ & $\begin{array}{c}\mathbf{0 . 8 0 8} \\
(34.399)\end{array}$ & $\begin{array}{c}\mathbf{0 . 6 6 0} \\
(19.107)\end{array}$ & $\begin{array}{c}0.692 \\
(34.414)\end{array}$ & $\begin{array}{c}\mathbf{0 . 8 2 2} \\
(26.606)\end{array}$ & $\begin{array}{c}\mathbf{0 . 6 0 0} \\
(13.239)\end{array}$ & $\begin{array}{c}\mathbf{0 . 6 4 8} \\
(24.789)\end{array}$ \\
\hline Superior Completa & $\begin{array}{c}0.845 \\
(29.033)\end{array}$ & $\begin{array}{c}\mathbf{0 . 5 2 0} \\
(13.577)\end{array}$ & $\begin{array}{c}0.727 \\
(29.329)\end{array}$ & $\begin{array}{c}0.891 \\
(26.008)\end{array}$ & $\begin{array}{c}0.559 \\
(10.286)\end{array}$ & $\begin{array}{c}\mathbf{0 . 6 3 3} \\
(20.302)\end{array}$ & $\begin{array}{c}\mathbf{0 . 8 7 4} \\
(30.050)\end{array}$ & $\begin{array}{c}\mathbf{0 . 6 4 4} \\
(17.166)\end{array}$ & $\begin{array}{c}\mathbf{0 . 7 4 2} \\
(29.976)\end{array}$ & $\begin{array}{c}0.987 \\
(28.288)\end{array}$ & $\begin{array}{c}\mathbf{0 . 6 1 7} \\
(11.207)\end{array}$ & $\begin{array}{c}\mathbf{0 . 6 5 5} \\
(20.857)\end{array}$ \\
\hline Experiencia Potencial & $\begin{array}{c}\mathbf{0 . 0 3 8} \\
(25.197)\end{array}$ & $\begin{array}{c}\mathbf{0 . 0 2 4} \\
(13.047)\end{array}$ & $\begin{array}{c}\mathbf{0 . 0 3 6} \\
(31.260)\end{array}$ & $\begin{array}{c}\mathbf{0 . 0 3 3} \\
(17.695)\end{array}$ & $\begin{array}{c}\mathbf{0 . 0 2 6} \\
(11.907)\end{array}$ & $\begin{array}{c}\mathbf{0 . 0 3 3} \\
(22.546)\end{array}$ & $\begin{array}{c}\mathbf{0 . 0 3 8} \\
(24.655)\end{array}$ & $\begin{array}{c}\mathbf{0 . 0 2 2} \\
(11.846)\end{array}$ & $\begin{array}{c}\mathbf{0 . 0 3 5} \\
(29.342)\end{array}$ & $\begin{array}{c}\mathbf{0 . 0 3 2} \\
(16.393)\end{array}$ & $\begin{array}{c}\mathbf{0 . 0 2 4} \\
(10.557)\end{array}$ & $\begin{array}{c}\mathbf{0 . 0 3 0} \\
(19.881)\end{array}$ \\
\hline Exper. Potencial 2 & $\begin{array}{c}-0.001 \\
(-20.995)\end{array}$ & $\begin{array}{c}\mathbf{0 . 0 0 0} \\
(-10.353)\end{array}$ & $\begin{array}{c}-0.001 \\
(-24.810)\end{array}$ & $\begin{array}{c}0.000 \\
(-13.184)\end{array}$ & $\begin{array}{c}0.000 \\
(-8.941)\end{array}$ & $\begin{array}{c}-0.001 \\
(-17.700)\end{array}$ & $\begin{array}{c}-0.001 \\
(-20.883)\end{array}$ & $\begin{array}{c}0.000 \\
(-9.273)\end{array}$ & $\begin{array}{c}-0.001 \\
(-23.624)\end{array}$ & $\begin{array}{c}0.000 \\
(-12.611)\end{array}$ & $\begin{array}{c}0.000 \\
(-8.413)\end{array}$ & $\begin{array}{c}\mathbf{0 . 0 0 0} \\
(-16.466)\end{array}$ \\
\hline Antigüedad & $\begin{array}{c}\mathbf{0 . 0 5 2} \\
(16.308)\end{array}$ & $\begin{array}{c}\mathbf{0 . 0 7 1} \\
(15.509)\end{array}$ & $\begin{array}{c}\mathbf{0 . 0 6 3} \\
(23.373)\end{array}$ & $\begin{array}{c}\mathbf{0 . 0 8 9} \\
(22.642)\end{array}$ & $\begin{array}{c}\mathbf{0 . 1 1 6} \\
(21.346)\end{array}$ & $\begin{array}{c}\mathbf{0 . 1 0 6} \\
(32.355)\end{array}$ & $\begin{array}{c}\mathbf{0 . 0 5 3} \\
(16.690)\end{array}$ & $\begin{array}{c}\mathbf{0 . 0 8 2} \\
(17.654)\end{array}$ & $\begin{array}{c}\mathbf{0 . 0 6 6} \\
(24.250)\end{array}$ & $\begin{array}{c}\mathbf{0 . 0 9 7} \\
(24.139)\end{array}$ & $\begin{array}{c}\mathbf{0 . 1 2 9} \\
(23.481)\end{array}$ & $\begin{array}{c}0.113 \\
(33.816)\end{array}$ \\
\hline Profesional & $\begin{array}{c}\mathbf{0 . 5 7 5} \\
(5.561)\end{array}$ & $\begin{array}{c}0.235 \\
(1.522)\end{array}$ & $\begin{array}{c}\mathbf{0 . 5 3 2} \\
(5.994)\end{array}$ & $\begin{array}{c}\mathbf{0 . 6 2 8} \\
(4.892)\end{array}$ & $\begin{array}{c}0.663 \\
(3.978)\end{array}$ & $\begin{array}{c}\mathbf{0 . 6 9 9} \\
(6.681)\end{array}$ & $\begin{array}{c}0.554 \\
(5.244)\end{array}$ & $\begin{array}{c}\mathbf{0 . 1 3 6} \\
(0.857)\end{array}$ & $\begin{array}{c}\mathbf{0 . 4 7 7} \\
(5.177)\end{array}$ & $\begin{array}{c}\mathbf{0 . 5 9 7} \\
(4.451)\end{array}$ & $\begin{array}{c}0.450 \\
(2.630)\end{array}$ & $\begin{array}{c}\mathbf{0 . 6 3 2} \\
(5.794)\end{array}$ \\
\hline Calificados & $\begin{array}{c}\mathbf{0 . 1 7 4} \\
(1.709)\end{array}$ & $\begin{array}{c}-0.219 \\
(-1.433)\end{array}$ & $\begin{array}{c}\mathbf{0 . 0 4 9} \\
(0.561)\end{array}$ & $\begin{array}{c}\mathbf{0 . 1 2 8} \\
(1.013)\end{array}$ & $\begin{array}{c}\mathbf{0 . 0 5 6} \\
(0.337)\end{array}$ & $\begin{array}{c}\mathbf{0 . 0 9 1} \\
(0.878)\end{array}$ & $\begin{array}{c}\mathbf{0 . 1 4 5} \\
(1.397)\end{array}$ & $\begin{array}{c}-0.302 \\
(-1.920)\end{array}$ & $\begin{array}{c}\mathbf{0 . 0 0 0} \\
(0.005)\end{array}$ & $\begin{array}{c}\mathbf{0 . 0 7 8} \\
(0.590)\end{array}$ & $\begin{array}{c}-0.146 \\
(-0.865)\end{array}$ & $\begin{array}{c}\mathbf{0 . 0 1 2} \\
(0.111)\end{array}$ \\
\hline No Calificado & $\begin{array}{c}\mathbf{0 . 0 1 2} \\
(0.114)\end{array}$ & $\begin{array}{c}-0.283 \\
(-1.844)\end{array}$ & $\begin{array}{c}-0.112 \\
(-1.274)\end{array}$ & $\begin{array}{c}-0.104 \\
(-0.818)\end{array}$ & $\begin{array}{c}-0.172 \\
(-1.039)\end{array}$ & $\begin{array}{c}-0.174 \\
(-1.678)\end{array}$ & $\begin{array}{c}-0.005 \\
(-0.048)\end{array}$ & $\begin{array}{c}-0.441 \\
(-2.795)\end{array}$ & $\begin{array}{c}-0.244 \\
(-2.681)\end{array}$ & $\begin{array}{c}-0.148 \\
(-1.116)\end{array}$ & $\begin{array}{c}-0.472 \\
(-2.785)\end{array}$ & $\begin{array}{c}-0.353 \\
(-3.270)\end{array}$ \\
\hline Lambda & $\begin{array}{c}-0.624 \\
(-10.858)\end{array}$ & $\begin{array}{c}-0.235 \\
(-6.376)\end{array}$ & $\begin{array}{c}-0.011 \\
(-0.259)\end{array}$ & $\begin{array}{c}-0.354 \\
(-13.408)\end{array}$ & $\begin{array}{c}-0.184 \\
(-4.675)\end{array}$ & $\begin{array}{c}-0.220 \\
(-7.225)\end{array}$ & $\begin{array}{c}-0.670 \\
(-11.418)\end{array}$ & $\begin{array}{c}-0.243 \\
(-6.402)\end{array}$ & $\begin{array}{c}-0.006 \\
(-0.139)\end{array}$ & $\begin{array}{c}-0.395 \\
(-14.364)\end{array}$ & $\begin{array}{c}-0.194 \\
(-4.767)\end{array}$ & $\begin{array}{c}-0.256 \\
(-8.048)\end{array}$ \\
\hline Primario & $\begin{array}{c}-0.022 \\
(-0.551)\end{array}$ & $\begin{array}{c}-0.084 \\
(-0.802)\end{array}$ & $\begin{array}{c}-0.006 \\
(-0.156)\end{array}$ & $\begin{array}{c}\mathbf{0 . 0 2 0} \\
(0.367)\end{array}$ & $\begin{array}{c}-0.357 \\
(-2.360)\end{array}$ & $\begin{array}{c}\mathbf{0 . 0 1 4} \\
(0.284)\end{array}$ & & & & & & \\
\hline Industria & $\begin{array}{c}-0.160 \\
(-4.978)\end{array}$ & $\begin{array}{c}-0.392 \\
(-7.724)\end{array}$ & $\begin{array}{c}-0.225 \\
(-8.050)\end{array}$ & $\begin{array}{c}-0.267 \\
(-6.243)\end{array}$ & $\begin{array}{c}-0.558 \\
(-9.183)\end{array}$ & $\begin{array}{c}-0.342 \\
(-9.542)\end{array}$ & & & & & & \\
\hline Sector Público & $\begin{array}{c}-0.100 \\
(-2.191)\end{array}$ & $\begin{array}{c}-0.202 \\
(-2.093)\end{array}$ & $\begin{array}{c}-0.117 \\
(-2.775)\end{array}$ & $\begin{array}{c}-0.109 \\
(-1.669)\end{array}$ & $\begin{array}{c}-0.147 \\
(-0.950)\end{array}$ & $\begin{array}{c}-0.093 \\
(-1.528)\end{array}$ & & & & & & \\
\hline Construcción & $\begin{array}{c}-0.283 \\
(-8.484)\end{array}$ & $\begin{array}{c}-0.154 \\
(-1.427)\end{array}$ & $\begin{array}{c}-0.267 \\
(-9.015)\end{array}$ & $\begin{array}{c}-0.346 \\
(-7.904)\end{array}$ & $\begin{array}{c}-0.315 \\
(-2.612)\end{array}$ & $\begin{array}{c}-0.330 \\
(-8.792)\end{array}$ & & & & & & \\
\hline Comercio & $\begin{array}{c}-0.124 \\
(-3.888)\end{array}$ & $\begin{array}{c}-0.236 \\
(-4.850)\end{array}$ & $\begin{array}{c}-0.168 \\
(-6.127)\end{array}$ & $\begin{array}{c}-0.194 \\
(-4.639)\end{array}$ & $\begin{array}{c}-0.392 \\
(-6.956)\end{array}$ & $\begin{array}{c}-0.263 \\
(-7.581)\end{array}$ & & & & & & \\
\hline Transporte & $\begin{array}{c}-0.037 \\
(-1.098)\end{array}$ & $\begin{array}{c}-0.092 \\
(-1.393)\end{array}$ & $\begin{array}{c}-0.029 \\
(-0.971)\end{array}$ & $\begin{array}{c}-0.141 \\
(-3.217)\end{array}$ & $\begin{array}{c}-0.165 \\
(-2.300)\end{array}$ & $\begin{array}{c}-0.125 \\
(-3.337)\end{array}$ & & & & & & \\
\hline Servicios Sociales & $\begin{array}{c}-0.241 \\
(-7.736)\end{array}$ & $\begin{array}{c}-0.381 \\
(-8.190)\end{array}$ & $\begin{array}{c}-0.370 \\
(-13.921)\end{array}$ & $\begin{array}{c}-0.290 \\
(-6.985)\end{array}$ & $\begin{array}{c}-0.463 \\
(-8.528)\end{array}$ & $\begin{array}{c}-0.416 \\
(-12.269)\end{array}$ & & & & & & \\
\hline Servicio Doméstico & $\begin{array}{c}-0.311 \\
(-6.743)\end{array}$ & $\begin{array}{c}-0.429 \\
(-8.206)\end{array}$ & $\begin{array}{c}-0.468 \\
(-15.073)\end{array}$ & $\begin{array}{c}-0.442 \\
(-7.612)\end{array}$ & $\begin{array}{c}-0.317 \\
(-5.172)\end{array}$ & $\begin{array}{c}-0.382 \\
(-9.762)\end{array}$ & & & & & & \\
\hline Tamaño 1 & $\begin{array}{c}-0.149 \\
(-8.415)\end{array}$ & $\begin{array}{c}-0.171 \\
(-5.905)\end{array}$ & $\begin{array}{c}-0.185 \\
(-11.897)\end{array}$ & $\begin{array}{c}-0.340 \\
(-13.470)\end{array}$ & $\begin{array}{c}-0.239 \\
(-6.457)\end{array}$ & $\begin{array}{c}-0.334 \\
(-15.607)\end{array}$ & & & & & & \\
\hline Tamaño 2 & $\begin{array}{c}-0.029 \\
(-1.720)\end{array}$ & $\begin{array}{c}\mathbf{0 . 0 2 0} \\
(0.730)\end{array}$ & $\begin{array}{c}-0.038 \\
(-2.546)\end{array}$ & $\begin{array}{c}-0.143 \\
(-6.050)\end{array}$ & $\begin{array}{c}\mathbf{0 . 0 7 6} \\
(2.214)\end{array}$ & $\begin{array}{c}-0.086 \\
(-4.300)\end{array}$ & & & & & & \\
\hline Tamaño 3 & $\begin{array}{c}\mathbf{0 . 0 3 9} \\
(2.311)\end{array}$ & $\begin{array}{c}\mathbf{0 . 0 6 9} \\
(2.707)\end{array}$ & $\begin{array}{c}\mathbf{0 . 0 1 6} \\
(1.063)\end{array}$ & $\begin{array}{c}-0.016 \\
(-0.689)\end{array}$ & $\begin{array}{c}0.192 \\
(5.703)\end{array}$ & $\begin{array}{c}\mathbf{0 . 0 4 3} \\
(2.145)\end{array}$ & & & & & & \\
\hline Tamaño 4 & $\begin{array}{c}0.093 \\
(5.116)\end{array}$ & $\begin{array}{c}0.085 \\
(3.182)\end{array}$ & $\begin{array}{c}\mathbf{0 . 0 6 5} \\
(4.194)\end{array}$ & $\begin{array}{c}\mathbf{0 . 1 0 8} \\
(4.235)\end{array}$ & $\begin{array}{c}\mathbf{0 . 2 0 6} \\
(5.901)\end{array}$ & $\begin{array}{c}\mathbf{0 . 1 2 1} \\
(5.718)\end{array}$ & & & & & & \\
\hline Tamaño 5 & $\begin{array}{c}\mathbf{0 . 1 3 1} \\
(6.287)\end{array}$ & $\begin{array}{c}\mathbf{0 . 1 8 6} \\
(5.857)\end{array}$ & $\begin{array}{c}0.142 \\
(7.880)\end{array}$ & $\begin{array}{c}\mathbf{0 . 1 4 2} \\
(5.086)\end{array}$ & $\begin{array}{c}\mathbf{0 . 2 9 9} \\
(7.379)\end{array}$ & $\begin{array}{c}0.209 \\
(8.788)\end{array}$ & & & & & & \\
\hline Tamaño 6 & $\begin{array}{c}0.093 \\
(3.569) \\
\end{array}$ & $\begin{array}{c}\mathbf{0 . 2 7 2} \\
(6.554) \\
\end{array}$ & $\begin{array}{r}\mathbf{0 . 1 6 1} \\
(7.052) \\
\end{array}$ & $\begin{array}{c}\mathbf{0 . 1 6 4} \\
(4.447) \\
\end{array}$ & $\begin{array}{c}\mathbf{0 . 2 6 6} \\
(5.129) \\
\end{array}$ & $\begin{array}{c}\mathbf{0 . 2 1 4} \\
(6.895) \\
\end{array}$ & & & & & & \\
\hline F-Stat & 393 & 204 & 567 & 361 & 231 & 558 & 680 & 334 & 896 & 574 & 386 & 879 \\
\hline Observaciones & 14962 & 9001 & 23963 & 10707 & 7296 & 18003 & 14962 & 9001 & 23963 & 10707 & 7296 & 18003 \\
\hline
\end{tabular}

Fuente: Elaboración propia en base a datos de la EPH. 


\section{Cuadro 12}

Ecuación del Log del Salario Horario, MCO ajustado por sesgo de selección Argentina, 1994-2000

\begin{tabular}{|c|c|c|c|c|c|c|c|c|c|c|c|c|}
\hline & \multicolumn{6}{|c|}{ Ecuación Completa } & \multicolumn{6}{|c|}{ Ecuación de Características Personales } \\
\hline & \multicolumn{3}{|c|}{1994} & \multicolumn{3}{|c|}{2000} & \multicolumn{3}{|c|}{1994} & \multicolumn{3}{|c|}{2000} \\
\hline & Hombre & Mujer & Total & Hombre & Mujer & Total & Hombre & Mujer & Total & Hombre & Mujer & Total \\
\hline Constante & $\begin{array}{c}\mathbf{0 . 4 1 5} \\
(0.664)\end{array}$ & $\begin{array}{c}2.327 \\
(3.683)\end{array}$ & $\begin{array}{c}\mathbf{0 . 7 3 6} \\
(1.622)\end{array}$ & $\begin{array}{c}\mathbf{0 . 1 1 3} \\
(0.830)\end{array}$ & $\begin{array}{c}-0.032 \\
(-0.187)\end{array}$ & $\begin{array}{c}\mathbf{0 . 0 8 3} \\
(0.766)\end{array}$ & $\begin{array}{c}-0.141 \\
(-0.223)\end{array}$ & $\begin{array}{c}1.666 \\
(2.615)\end{array}$ & $\begin{array}{c}\mathbf{0 . 0 9 7} \\
(0.212)\end{array}$ & $\begin{array}{c}-0.244 \\
(-1.809)\end{array}$ & $\begin{array}{c}-0.199 \\
(-1.168)\end{array}$ & $\begin{array}{c}-0.273 \\
(-2.538)\end{array}$ \\
\hline Primaria Completa & $\begin{array}{c}\mathbf{0 . 1 4 6} \\
(3.445)\end{array}$ & $\begin{array}{c}-0.036 \\
(-0.594)\end{array}$ & $\begin{array}{c}\mathbf{0 . 1 0 0} \\
(2.873)\end{array}$ & $\begin{array}{c}\mathbf{0 . 1 3 0} \\
(5.317)\end{array}$ & $\begin{array}{c}\mathbf{0 . 0 5 3} \\
(1.607)\end{array}$ & $\begin{array}{c}\mathbf{0 . 1 0 0} \\
(5.039)\end{array}$ & $\begin{array}{c}\mathbf{0 . 1 8 5} \\
(4.335)\end{array}$ & $\begin{array}{c}-0.065 \\
(-1.043)\end{array}$ & $\begin{array}{c}0.109 \\
(3.088)\end{array}$ & $\begin{array}{c}\mathbf{0 . 1 4 3} \\
(5.718)\end{array}$ & $\begin{array}{c}\mathbf{0 . 0 2 2} \\
(0.630)\end{array}$ & $\begin{array}{c}\mathbf{0 . 1 0 4} \\
(5.147)\end{array}$ \\
\hline Secundaria Incompleta & $\begin{array}{c}\mathbf{0 . 2 5 2} \\
(5.473)\end{array}$ & $\begin{array}{c}\mathbf{0 . 0 8 9} \\
(1.328)\end{array}$ & $\begin{array}{c}\mathbf{0 . 2 2 3} \\
(5.769)\end{array}$ & $\begin{array}{c}\mathbf{0 . 2 7 4} \\
(10.576)\end{array}$ & $\begin{array}{c}\mathbf{0 . 0 8 6} \\
(2.363)\end{array}$ & $\begin{array}{c}\mathbf{0 . 2 0 4} \\
(9.530)\end{array}$ & $\begin{array}{c}\mathbf{0 . 3 1 7} \\
(6.901)\end{array}$ & $\begin{array}{c}\mathbf{0 . 0 5 4} \\
(0.796)\end{array}$ & $\begin{array}{c}\mathbf{0 . 2 4 9} \\
(6.449)\end{array}$ & $\begin{array}{c}\mathbf{0 . 2 9 9} \\
(11.357)\end{array}$ & $\begin{array}{c}\mathbf{0 . 0 4 2} \\
(1.113)\end{array}$ & $\begin{array}{c}\mathbf{0 . 2 1 8} \\
(10.041)\end{array}$ \\
\hline Secundaria Completa & $\begin{array}{c}\mathbf{0 . 4 4 2} \\
(9.040)\end{array}$ & $\begin{array}{c}0.303 \\
(4.158)\end{array}$ & $\begin{array}{c}0.405 \\
(9.931)\end{array}$ & $\begin{array}{c}\mathbf{0 . 4 8 2} \\
(17.766)\end{array}$ & $\begin{array}{c}\mathbf{0 . 3 6 2} \\
(9.350)\end{array}$ & $\begin{array}{c}\mathbf{0 . 4 1 9} \\
(18.469)\end{array}$ & $\begin{array}{c}0.535 \\
(11.082)\end{array}$ & $\begin{array}{c}\mathbf{0 . 3 0 9} \\
(4.288)\end{array}$ & $\begin{array}{c}\mathbf{0 . 4 6 9} \\
(11.761)\end{array}$ & $\begin{array}{c}0.541 \\
(19.783)\end{array}$ & $\begin{array}{c}0.313 \\
(7.878)\end{array}$ & $\begin{array}{c}\mathbf{0 . 4 6 5} \\
(20.377)\end{array}$ \\
\hline Superior Incompleta & $\begin{array}{c}\mathbf{0 . 6 8 2} \\
(11.395)\end{array}$ & $\begin{array}{c}\mathbf{0 . 4 9 1} \\
(5.634)\end{array}$ & $\begin{array}{c}\mathbf{0 . 6 1 1} \\
(12.586)\end{array}$ & $\begin{array}{c}\mathbf{0 . 6 7 1} \\
(21.998)\end{array}$ & $\begin{array}{c}\mathbf{0 . 4 6 9} \\
(11.317)\end{array}$ & $\begin{array}{c}0.576 \\
(23.513)\end{array}$ & $\begin{array}{c}\mathbf{0 . 8 1 2} \\
(13.790)\end{array}$ & $\begin{array}{c}\mathbf{0 . 5 4 7} \\
(6.439)\end{array}$ & $\begin{array}{c}\mathbf{0 . 7 1 8} \\
(15.150)\end{array}$ & $\begin{array}{c}\mathbf{0 . 7 4 8} \\
(24.456)\end{array}$ & $\begin{array}{c}\mathbf{0 . 4 6 0} \\
(10.822)\end{array}$ & $\begin{array}{c}\mathbf{0 . 6 4 3} \\
(26.219)\end{array}$ \\
\hline Superior Completa & $\begin{array}{c}\mathbf{0 . 8 2 1} \\
(11.122)\end{array}$ & $\begin{array}{c}\mathbf{0 . 6 5 0} \\
(6.805)\end{array}$ & $\begin{array}{c}\mathbf{0 . 8 2 7} \\
(13.690)\end{array}$ & $\begin{array}{c}\mathbf{0 . 8 4 7} \\
(24.530)\end{array}$ & $\begin{array}{c}0.754 \\
(14.936)\end{array}$ & $\begin{array}{c}\mathbf{0 . 7 5 2} \\
(25.240)\end{array}$ & $\begin{array}{c}0.976 \\
(13.327)\end{array}$ & $\begin{array}{c}\mathbf{0 . 7 0 5} \\
(7.560)\end{array}$ & $\begin{array}{c}0.965 \\
(16.356)\end{array}$ & $\begin{array}{c}\mathbf{0 . 9 6 2} \\
(27.856)\end{array}$ & $\begin{array}{c}\mathbf{0 . 7 9 8} \\
(15.457)\end{array}$ & $\begin{array}{c}0.892 \\
(30.245)\end{array}$ \\
\hline Experiencia Potencial & $\begin{array}{c}\mathbf{0 . 0 2 8} \\
(7.166)\end{array}$ & $\begin{array}{c}\mathbf{0 . 0 1 7} \\
(3.990)\end{array}$ & $\begin{array}{c}\mathbf{0 . 0 2 6} \\
(9.252)\end{array}$ & $\begin{array}{c}\mathbf{0 . 0 2 2} \\
(11.354)\end{array}$ & $\begin{array}{c}0.015 \\
(7.388)\end{array}$ & $\begin{array}{c}\mathbf{0 . 0 2 0} \\
(14.696)\end{array}$ & $\begin{array}{c}0.032 \\
(8.047)\end{array}$ & $\begin{array}{c}\mathbf{0 . 0 2 0} \\
(4.487)\end{array}$ & $\begin{array}{c}\mathbf{0 . 0 2 9} \\
(10.226)\end{array}$ & $\begin{array}{c}\mathbf{0 . 0 2 3} \\
(11.580)\end{array}$ & $\begin{array}{c}\mathbf{0 . 0 1 9} \\
(9.117)\end{array}$ & $\begin{array}{c}\mathbf{0 . 0 2 3} \\
(16.199)\end{array}$ \\
\hline Exper. Potencial 2 & $\begin{array}{c}\mathbf{0 . 0 0 0} \\
(-6.113)\end{array}$ & $\begin{array}{c}\mathbf{0 . 0 0 0} \\
(-3.322)\end{array}$ & $\begin{array}{c}\mathbf{0 . 0 0 0} \\
(-7.505)\end{array}$ & $\begin{array}{c}\mathbf{0 . 0 0 0} \\
(-7.413)\end{array}$ & $\begin{array}{c}\mathbf{0 . 0 0 0} \\
(-5.150)\end{array}$ & $\begin{array}{c}0.000 \\
(-10.398)\end{array}$ & $\begin{array}{c}-0.001 \\
(-6.886)\end{array}$ & $\begin{array}{c}0.000 \\
(-3.866)\end{array}$ & $\begin{array}{c}\mathbf{0 . 0 0 0} \\
(-8.326)\end{array}$ & $\begin{array}{c}\mathbf{0 . 0 0 0} \\
(-7.975)\end{array}$ & $\begin{array}{c}\mathbf{0 . 0 0 0} \\
(-7.185)\end{array}$ & $\begin{array}{c}\mathbf{0 . 0 0 0} \\
(-12.033)\end{array}$ \\
\hline Antigüedad & $\begin{array}{c}\mathbf{0 . 0 5 1} \\
(6.276)\end{array}$ & $\begin{array}{c}\mathbf{0 . 0 5 7} \\
(4.996)\end{array}$ & $\begin{array}{c}0.055 \\
(8.316)\end{array}$ & $\begin{array}{c}\mathbf{0 . 0 7 6} \\
(19.346)\end{array}$ & $\begin{array}{c}\mathbf{0 . 0 8 2} \\
(16.211)\end{array}$ & $\begin{array}{c}\mathbf{0 . 0 8 1} \\
(26.048)\end{array}$ & $\begin{array}{c}\mathbf{0 . 0 6 3} \\
(7.742)\end{array}$ & $\begin{array}{c}\mathbf{0 . 0 7 0} \\
(6.048)\end{array}$ & $\begin{array}{c}0.065 \\
(9.804)\end{array}$ & $\begin{array}{c}\mathbf{0 . 0 9 0} \\
(22.740)\end{array}$ & $\begin{array}{c}0.097 \\
(18.842)\end{array}$ & $\begin{array}{c}0.093 \\
(29.604)\end{array}$ \\
\hline Profesional & $\begin{array}{c}0.581 \\
(1.021)\end{array}$ & $\begin{array}{c}-1.673 \\
(-2.881)\end{array}$ & $\begin{array}{c}-0.481 \\
(-1.182)\end{array}$ & $\begin{array}{c}\mathbf{0 . 4 3 3} \\
(3.345)\end{array}$ & $\begin{array}{c}0.409 \\
(2.643)\end{array}$ & $\begin{array}{c}\mathbf{0 . 4 4 8} \\
(4.488)\end{array}$ & $\begin{array}{c}0.515 \\
(0.886)\end{array}$ & $\begin{array}{c}-1.415 \\
(-2.359)\end{array}$ & $\begin{array}{c}-0.426 \\
(-1.024)\end{array}$ & $\begin{array}{c}0.391 \\
(2.948)\end{array}$ & $\begin{array}{c}0.299 \\
(1.864)\end{array}$ & $\begin{array}{c}0.379 \\
(3.701)\end{array}$ \\
\hline Calificados & $\begin{array}{c}\mathbf{0 . 2 3 9} \\
(0.423)\end{array}$ & $\begin{array}{c}-2.040 \\
(-3.525)\end{array}$ & $\begin{array}{c}-0.858 \\
(-2.118)\end{array}$ & $\begin{array}{l}-0.020 \\
(-0.156)\end{array}$ & $\begin{array}{c}-0.038 \\
(-0.249)\end{array}$ & $\begin{array}{c}-0.023 \\
(-0.234)\end{array}$ & $\begin{array}{c}\mathbf{0 . 1 2 5} \\
(0.216)\end{array}$ & $\begin{array}{l}-1.800 \\
(-3.009)\end{array}$ & $\begin{array}{c}-0.828 \\
(-1.996)\end{array}$ & $\begin{array}{c}-0.077 \\
(-0.589)\end{array}$ & $\begin{array}{c}-0.115 \\
(-0.724)\end{array}$ & $\begin{array}{l}-0.081 \\
(-0.805)\end{array}$ \\
\hline No Calificado & $\begin{array}{c}\mathbf{0 . 0 3 1} \\
(0.054)\end{array}$ & $\begin{array}{c}-2.193 \\
(-3.789)\end{array}$ & $\begin{array}{c}-1.055 \\
(-2.603)\end{array}$ & $\begin{array}{c}-0.213 \\
(-1.668)\end{array}$ & $\begin{array}{c}-0.321 \\
(-2.085)\end{array}$ & $\begin{array}{c}-0.253 \\
(-2.558)\end{array}$ & $\begin{array}{l}-0.060 \\
(-0.103)\end{array}$ & $\begin{array}{c}-1.933 \\
(-3.230)\end{array}$ & $\begin{array}{c}-0.988 \\
(-2.382)\end{array}$ & $\begin{array}{c}-0.286 \\
(-2.188)\end{array}$ & $\begin{array}{c}-0.388 \\
(-2.440)\end{array}$ & $\begin{array}{c}-0.311 \\
(-3.072)\end{array}$ \\
\hline Lambda & $\begin{array}{c}-0.174 \\
(-1.209)\end{array}$ & $\begin{array}{c}\mathbf{0 . 1 7 9} \\
(1.972)\end{array}$ & $\begin{array}{c}0.317 \\
(3.133)\end{array}$ & $\begin{array}{c}-0.087 \\
(-3.288)\end{array}$ & $\begin{array}{c}\mathbf{0 . 2 1 0} \\
(5.738)\end{array}$ & $\begin{array}{c}\mathbf{0 . 0 3 2} \\
(1.094)\end{array}$ & $\begin{array}{c}-0.082 \\
(-0.564)\end{array}$ & $\begin{array}{c}\mathbf{0 . 1 5 0} \\
(1.617)\end{array}$ & $\begin{array}{c}0.373 \\
(3.613)\end{array}$ & $\begin{array}{c}-0.081 \\
(-2.992)\end{array}$ & $\begin{array}{c}\mathbf{0 . 2 1 6} \\
(5.679)\end{array}$ & $\begin{array}{c}\mathbf{0 . 0 8 6} \\
(2.872)\end{array}$ \\
\hline Primario & $\begin{array}{c}-0.534 \\
(-4.833)\end{array}$ & $\begin{array}{c}-0.180 \\
(-0.572)\end{array}$ & $\begin{array}{c}-0.528 \\
(-5.223)\end{array}$ & $\begin{array}{c}-0.125 \\
(-2.282)\end{array}$ & $\begin{array}{c}-0.498 \\
(-3.543)\end{array}$ & $\begin{array}{c}-0.129 \\
(-2.711)\end{array}$ & & & & & & \\
\hline Industria & $\begin{array}{c}-0.294 \\
(-3.578)\end{array}$ & $\begin{array}{c}-0.616 \\
(-4.565)\end{array}$ & $\begin{array}{c}-0.386 \\
(-5.468)\end{array}$ & $\begin{array}{c}-0.335 \\
(-7.759)\end{array}$ & $\begin{array}{c}-0.432 \\
(-7.654)\end{array}$ & $\begin{array}{c}-0.353 \\
(-10.321)\end{array}$ & & & & & & \\
\hline Sector Público & $\begin{array}{c}-0.005 \\
(-0.040)\end{array}$ & $\begin{array}{c}-0.257 \\
(-1.021)\end{array}$ & $\begin{array}{c}-0.047 \\
(-0.462)\end{array}$ & $\begin{array}{c}-0.055 \\
(-0.837)\end{array}$ & $\begin{array}{c}\mathbf{0 . 0 6 5} \\
(0.455)\end{array}$ & $\begin{array}{c}-0.013 \\
(-0.218)\end{array}$ & & & & & & \\
\hline Construcción & $\begin{array}{c}-0.396 \\
(-4.664)\end{array}$ & $\begin{array}{c}-0.045 \\
(-0.155)\end{array}$ & $\begin{array}{c}-0.410 \\
(-5.539)\end{array}$ & $\begin{array}{c}-0.335 \\
(-7.592)\end{array}$ & $\begin{array}{c}-0.200 \\
(-1.781)\end{array}$ & $\begin{array}{c}-0.317 \\
(-8.867)\end{array}$ & & & & & & \\
\hline Comercio & $\begin{array}{c}-0.295 \\
(-3.611)\end{array}$ & $\begin{array}{c}-0.485 \\
(-3.680)\end{array}$ & $\begin{array}{c}-0.354 \\
(-5.093)\end{array}$ & $\begin{array}{c}-0.339 \\
(-8.031)\end{array}$ & $\begin{array}{c}-0.391 \\
(-7.475)\end{array}$ & $\begin{array}{c}-0.362 \\
(-10.934)\end{array}$ & & & & & & \\
\hline Transporte & $\begin{array}{c}-0.255 \\
(-2.943)\end{array}$ & $\begin{array}{c}-0.242 \\
(-1.293)\end{array}$ & $\begin{array}{l}-0.265 \\
(-3.486)\end{array}$ & $\begin{array}{l}-0.320 \\
(-7.224)\end{array}$ & $\begin{array}{l}-0.223 \\
(-3.333)\end{array}$ & $\begin{array}{c}-0.290 \\
(-8.090)\end{array}$ & & & & & & \\
\hline Servicios Sociales & $\begin{array}{c}-0.150 \\
(-1.888)\end{array}$ & $\begin{array}{c}-0.260 \\
(-2.055)\end{array}$ & $\begin{array}{c}-0.204 \\
(-3.052)\end{array}$ & $\begin{array}{c}-0.172 \\
(-4.106)\end{array}$ & $\begin{array}{c}-0.122 \\
(-2.419)\end{array}$ & $\begin{array}{c}-0.168 \\
(-5.205)\end{array}$ & & & & & & \\
\hline Servicio Doméstico & $\begin{array}{c}-0.316 \\
(-2.820)\end{array}$ & $\begin{array}{c}-0.151 \\
(-1.103)\end{array}$ & $\begin{array}{c}-0.154 \\
(-2.023)\end{array}$ & $\begin{array}{c}-0.306 \\
(-5.231)\end{array}$ & $\begin{array}{c}0.064 \\
(1.124)\end{array}$ & $\begin{array}{c}-0.056 \\
(-1.504)\end{array}$ & & & & & & \\
\hline Tamaño 1 & $\begin{array}{l}-0.125 \\
(-2.815)\end{array}$ & $\begin{array}{c}-0.162 \\
(-2.254)\end{array}$ & $\begin{array}{c}-0.142 \\
(-3.749)\end{array}$ & $\begin{array}{c}-0.137 \\
(-5.411)\end{array}$ & $\begin{array}{c}-0.104 \\
(-3.035)\end{array}$ & $\begin{array}{c}-0.129 \\
(-6.280)\end{array}$ & & & & & & \\
\hline Tamaño 2 & $\begin{array}{c}-0.192 \\
(-4.232)\end{array}$ & $\begin{array}{c}-0.132 \\
(-1.802)\end{array}$ & $\begin{array}{c}-0.179 \\
(-4.615)\end{array}$ & $\begin{array}{c}-0.101 \\
(-4.226)\end{array}$ & $\begin{array}{c}-0.029 \\
(-0.902)\end{array}$ & $\begin{array}{c}-0.079 \\
(-4.131)\end{array}$ & & & & & & \\
\hline Tamaño 3 & $\begin{array}{c}-0.044 \\
(-0.966)\end{array}$ & $\begin{array}{c}-0.004 \\
(-0.061)\end{array}$ & $\begin{array}{c}-0.047 \\
(-1.239)\end{array}$ & $\begin{array}{c}-0.012 \\
(-0.482)\end{array}$ & $\begin{array}{c}\mathbf{0 . 1 1 4} \\
(3.647)\end{array}$ & $\begin{array}{c}0.034 \\
(1.773)\end{array}$ & & & & & & \\
\hline Tamaño 4 & $\begin{array}{c}-0.033 \\
(-0.699)\end{array}$ & $\begin{array}{c}-0.003 \\
(-0.041)\end{array}$ & $\begin{array}{c}-0.034 \\
(-0.858)\end{array}$ & $\begin{array}{c}0.093 \\
(3.642)\end{array}$ & $\begin{array}{c}\mathbf{0 . 1 4 4} \\
(4.426)\end{array}$ & $\begin{array}{c}\mathbf{0 . 1 0 9} \\
(5.386)\end{array}$ & & & & & & \\
\hline Tamaño 5 & $\begin{array}{c}-0.050 \\
(-0.947)\end{array}$ & $\begin{array}{c}\mathbf{0 . 0 6 7} \\
(0.818)\end{array}$ & $\begin{array}{c}-0.015 \\
(-0.347)\end{array}$ & $\begin{array}{c}\mathbf{0 . 1 4 8} \\
(5.282)\end{array}$ & $\begin{array}{c}\mathbf{0 . 1 0 0} \\
(2.654)\end{array}$ & $\begin{array}{c}\mathbf{0 . 1 3 5} \\
(5.966)\end{array}$ & & & & & & \\
\hline Tamaño 6 & $\begin{array}{c}\mathbf{0 . 0 1 7} \\
(0.271) \\
\end{array}$ & $\begin{array}{c}\mathbf{0 . 1 2 3} \\
(1.245) \\
\end{array}$ & $\begin{array}{c}0.047 \\
(0.894) \\
\end{array}$ & $\begin{array}{c}\mathbf{0 . 1 3 0} \\
(3.499) \\
\end{array}$ & $\begin{array}{c}\mathbf{0 . 0 6 3} \\
(1.295) \\
\end{array}$ & $\begin{array}{c}0.110 \\
(3.722) \\
\end{array}$ & & & & & & \\
\hline F-Stat & 67 & 36 & 99 & 271 & 209 & 461 & 114 & 57 & 168 & 455 & 330 & 773 \\
\hline Observaciones & 2554 & 1515 & 4069 & 10705 & 7296 & 18001 & 2554 & 1515 & 4069 & 10705 & 7296 & 18001 \\
\hline
\end{tabular}

Fuente: Elaboración propia en base a datos de la EPH. 


\section{Cuadro 13}

Tamaño y estructura de las brechas de ingreso mensual y salario horario Argentina, 1994 - 2000

\begin{tabular}{|c|c|c|c|c|c|}
\hline & $\begin{array}{c}\text { Ventaja Hombes } \\
\text { (a) }\end{array}$ & $\begin{array}{c}\text { Desventaja } \\
\text { Mujeres } \\
\text { (b) }\end{array}$ & $\begin{array}{l}\text { Discriminación } \\
(\mathrm{c})=(\mathrm{a})+(\mathrm{b})\end{array}$ & $\begin{array}{c}\text { Características } \\
\text { (d) }\end{array}$ & $\begin{array}{l}\text { Brecha } \\
(\mathrm{c})+(\mathrm{d})\end{array}$ \\
\hline \multicolumn{6}{|c|}{ Ecuación Completa } \\
\hline \multicolumn{6}{|c|}{ Salario Horario } \\
\hline 1994 & $\begin{array}{c}0.076 \\
(0.017)\end{array}$ & $\begin{array}{c}0.092 \\
(0.018)\end{array}$ & $\begin{array}{c}0.169 \\
(0.038)\end{array}$ & $\begin{array}{c}-0.157 \\
(0.028)\end{array}$ & $\begin{array}{c}0.012 \\
(0.023)\end{array}$ \\
\hline 2000 & $\begin{array}{c}0.037 \\
(0.009)\end{array}$ & $\begin{array}{c}0.048 \\
(0.008)\end{array}$ & $\begin{array}{c}0.085 \\
(0.018)\end{array}$ & $\begin{array}{c}-0.097 \\
(0.015)\end{array}$ & $\begin{array}{c}-0.012 \\
(0.012)\end{array}$ \\
\hline \multicolumn{6}{|c|}{ Ingreso Mensual } \\
\hline 1994 & $\begin{array}{c}0.080 \\
(0.012)\end{array}$ & $\begin{array}{c}0.138 \\
(0.012)\end{array}$ & $\begin{array}{c}0.217 \\
(0.026)\end{array}$ & $\begin{array}{c}0.116 \\
(0.024)\end{array}$ & $\begin{array}{c}0.333 \\
(0.013)\end{array}$ \\
\hline 2000 & $\begin{array}{c}0.020 \\
(0.010)\end{array}$ & $\begin{array}{c}0.080 \\
(0.009)\end{array}$ & $\begin{array}{c}0.100 \\
(0.020)\end{array}$ & $\begin{array}{c}0.220 \\
(0.024)\end{array}$ & $\begin{array}{c}0.320 \\
(0.018)\end{array}$ \\
\hline \multicolumn{6}{|c|}{ Ecuación de Características Personales } \\
\hline \multicolumn{6}{|c|}{ Salario Horario } \\
\hline 1994 & $\begin{array}{c}0.068 \\
(0.019)\end{array}$ & $\begin{array}{c}0.070 \\
(0.021)\end{array}$ & $\begin{array}{c}0.138 \\
(0.042)\end{array}$ & $\begin{array}{l}-0.132 \\
(0.030)\end{array}$ & $\begin{array}{c}0.006 \\
(0.023)\end{array}$ \\
\hline 2000 & $\begin{array}{c}0.033 \\
(0.009)\end{array}$ & $\begin{array}{c}0.029 \\
(0.008)\end{array}$ & $\begin{array}{c}0.062 \\
(0.017)\end{array}$ & $\begin{array}{c}-0.077 \\
(0.016)\end{array}$ & $\begin{array}{c}-0.015 \\
(0.012)\end{array}$ \\
\hline \multicolumn{6}{|c|}{ Ingreso Mensual } \\
\hline 1994 & $\begin{array}{c}0.120 \\
(0.013)\end{array}$ & $\begin{array}{c}0.208 \\
(0.014)\end{array}$ & $\begin{array}{c}0.328 \\
(0.031)\end{array}$ & $\begin{array}{c}0.013 \\
(0.023)\end{array}$ & $\begin{array}{c}0.341 \\
(0.014)\end{array}$ \\
\hline 2000 & $\begin{array}{c}0.040 \\
(0.011) \\
\end{array}$ & $\begin{array}{c}0.120 \\
(0.010) \\
\end{array}$ & $\begin{array}{c}0.160 \\
(0.023) \\
\end{array}$ & $\begin{array}{c}0.154 \\
(0.023) \\
\end{array}$ & $\begin{array}{c}0.314 \\
(0.018) \\
\end{array}$ \\
\hline
\end{tabular}

Fuente: Elaboración propia en base a datos de la EPH. Nota: entre paréntesis se presentan los errores estándar (bootstrap). 


\section{Cuadro 14}

\section{Estructura vertical de la discriminación salarial}

Argentina, 1994 - 2000

\begin{tabular}{|c|c|c|c|c|c|c|c|c|c|c|c|c|c|c|c|c|}
\hline \multirow{3}{*}{$\begin{array}{c}\text { Ingreso laboral } \\
\text { Horario }\end{array}$} & \multicolumn{8}{|c|}{ Modelo Completo } & \multicolumn{8}{|c|}{ Modelo Características Personales } \\
\hline & \multicolumn{4}{|c|}{1994} & \multicolumn{4}{|c|}{2000} & \multicolumn{4}{|c|}{1994} & \multicolumn{4}{|c|}{2000} \\
\hline & $\mathrm{HO}$ & $\mathrm{MU}$ & $\% \mathrm{HO}$ & $\% \mathrm{MU}$ & $\mathrm{HO}$ & $\mathrm{MU}$ & $\% \mathrm{HO}$ & $\% \mathrm{MU}$ & $\mathrm{HO}$ & $\mathrm{MU}$ & $\% \mathrm{HO}$ & $\% \mathrm{MU}$ & $\mathrm{HO}$ & $\mathrm{MU}$ & $\% \mathrm{HO}$ & $\% \mathrm{MU}$ \\
\hline Educación & 0.16 & 0.00 & 6 & 0 & 0.11 & 0.00 & 28 & 0 & 0.24 & 0.00 & 10 & 0 & 0.08 & 0.00 & 31 & 0 \\
\hline Experiencia & 0.12 & 0.00 & 5 & 0 & 0.10 & 0.00 & 24 & 0 & 0.14 & 0.00 & 6 & 0 & 0.08 & 0.00 & 30 & 0 \\
\hline Antigüedad & 0.00 & 0.03 & 0 & 1 & 0.00 & 0.02 & 0 & 7 & 0.00 & 0.03 & 0 & 1 & 0.00 & 0.02 & 0 & 6 \\
\hline Tipo de Trabajador & 2.25 & 0.00 & 85 & 0 & 0.05 & 0.00 & 12 & 0 & 1.90 & 0.00 & 83 & 0 & 0.11 & 0.00 & 40 & 0 \\
\hline Región & 0.00 & 0.00 & 0 & 0 & 0.00 & 0.02 & 0 & 8 & 0.00 & 0.00 & 0 & 0 & 0.00 & 0.02 & 0 & 7 \\
\hline Sector Productivo & 0.11 & 0.00 & 4 & 0 & 0.00 & 0.04 & 0 & 11 & - & - & - & - & - & - & - & - \\
\hline Tamaño de la Firma & 0.00 & 0.02 & 0 & 1 & 0.00 & 0.05 & 0 & 15 & - & - & - & - & - & - & - & - \\
\hline Lambda & 0.00 & 0.51 & 0 & 21 & 0.00 & 0.19 & 0 & 59 & 0.00 & 0.30 & 0 & 14 & 0.00 & 0.21 & 0 & 71 \\
\hline Constante & 0.00 & 1.91 & 0 & 77 & 0.15 & 0.00 & 36 & 0 & 0.00 & 1.81 & 0 & 84 & 0.00 & 0.05 & 0 & 16 \\
\hline Total & 2.64 & 2.48 & 100 & 100 & 0.40 & 0.32 & 100 & 100 & 2.28 & 2.15 & 100 & 100 & 0.27 & 0.29 & 100 & 100 \\
\hline \multirow{3}{*}{ Ingreso Mensual } & \multicolumn{8}{|c|}{ Modelo Completo } & \multicolumn{8}{|c|}{ Modelo Características Personales } \\
\hline & \multicolumn{4}{|c|}{1994} & \multicolumn{4}{|c|}{2000} & \multicolumn{4}{|c|}{1994} & \multicolumn{4}{|c|}{2000} \\
\hline & $\mathrm{HO}$ & $\mathrm{MU}$ & $\% \mathrm{HO}$ & $\% \mathrm{MU}$ & $\mathrm{HO}$ & $\mathrm{MU}$ & $\% \mathrm{HO}$ & $\% \mathrm{MU}$ & $\mathrm{HO}$ & $\mathrm{MU}$ & $\% \mathrm{HO}$ & $\% \mathrm{MU}$ & $\mathrm{HO}$ & $\mathrm{MU}$ & $\% \mathrm{HO}$ & $\% \mathrm{MU}$ \\
\hline Educación & 0.14 & 0.00 & 17 & 0 & 0.14 & 0.00 & 32 & 0 & 0.07 & 0.00 & 7 & 0 & 0.09 & 0.00 & 16 & 0 \\
\hline Experiencia & 0.17 & 0.00 & 20 & 0 & 0.10 & 0.00 & 22 & 0 & 0.18 & 0.00 & 17 & 0 & 0.13 & 0.00 & 23 & 0 \\
\hline Antigüedad & 0.00 & 0.08 & 0 & 13 & 0.00 & 0.11 & 0 & 34 & 0.00 & 0.12 & 0 & 17 & 0.00 & 0.12 & 0 & 46 \\
\hline Tipo de Trabajador & 0.34 & 0.00 & 40 & 0 & 0.06 & 0.00 & 13 & 0 & 0.42 & 0.00 & 41 & 0 & 0.30 & 0.00 & 54 & 0 \\
\hline Región & 0.01 & 0.00 & 1 & 0 & 0.03 & 0.00 & 6 & 0 & 0.01 & 0.00 & 1 & 0 & 0.03 & 0.00 & 5 & 0 \\
\hline Sector Productivo & 0.08 & 0.00 & 9 & 0 & 0.11 & 0.00 & 25 & 0 & - & - & - & - & - & - & - & - \\
\hline Tamaño de la Firma & 0.00 & 0.02 & 0 & 3 & 0.00 & 0.15 & 0 & 43 & - & - & - & - & - & - & - & - \\
\hline Lambda & 0.00 & 0.55 & 0 & 84 & 0.00 & 0.08 & 0 & 23 & 0.00 & 0.60 & 0 & 83 & 0.02 & 0.00 & 3 & 0 \\
\hline Constante & 0.12 & 0.00 & 13 & 0 & 0.01 & 0.00 & 2 & 0 & 0.35 & 0.00 & 34 & 0 & 0.00 & 0.14 & 0 & 54 \\
\hline Total & 0.86 & 0.65 & 100 & 100 & 0.44 & 0.34 & 100 & 100 & 1.03 & 0.73 & 100 & 100 & 0.56 & 0.26 & 100 & 100 \\
\hline
\end{tabular}

Fuente: Elaboración propia en base a datos de la EPH. 


\section{Cuadro 15}

Ecuación para la probabilidad de estar desempleado (Modelo Probit) Argentina, 1994-2000

\begin{tabular}{|c|c|c|c|c|c|c|c|c|c|c|c|c|}
\hline & \multicolumn{6}{|c|}{ Ecuación Completa } & \multicolumn{6}{|c|}{ Ecuación de Características Personales } \\
\hline & \multicolumn{3}{|c|}{1994} & \multicolumn{3}{|c|}{2000} & \multicolumn{3}{|c|}{1994} & \multicolumn{3}{|c|}{2000} \\
\hline & Hombre & Mujer & Total & Hombre & Mujer & Total & Hombre & Mujer & Total & Hombre & Mujer & Total \\
\hline Constante & $\begin{array}{c}1.811 \\
(12.681)\end{array}$ & $\begin{array}{c}1.305 \\
(10.599)\end{array}$ & $\begin{array}{c}1.565 \\
(17.300)\end{array}$ & $\begin{array}{c}1.790 \\
(12.577)\end{array}$ & $\begin{array}{c}1.228 \\
(10.107)\end{array}$ & $\begin{array}{c}1.489 \\
(16.437)\end{array}$ & $\begin{array}{c}1.848 \\
(13.507)\end{array}$ & $\begin{array}{c}1.319 \\
(11.338)\end{array}$ & $\begin{array}{c}1.608 \\
(18.623)\end{array}$ & $\begin{array}{c}1.819 \\
(13.167)\end{array}$ & $\begin{array}{c}1.400 \\
(11.981)\end{array}$ & $\begin{array}{c}1.633 \\
(18.653)\end{array}$ \\
\hline Primaria Completa & $\begin{array}{c}\mathbf{0 . 0 1 2} \\
(0.161)\end{array}$ & $\begin{array}{c}-0.049 \\
(-0.949)\end{array}$ & $\begin{array}{l}-0.023 \\
(-0.559)\end{array}$ & $\begin{array}{c}-0.096 \\
(-1.281)\end{array}$ & $\begin{array}{c}-\mathbf{0 . 0 3 0} \\
(-0.550)\end{array}$ & $\begin{array}{c}-0.058 \\
(-1.311)\end{array}$ & $\begin{array}{c}-0.002 \\
(-0.021)\end{array}$ & $\begin{array}{c}-0.117 \\
(-2.312)\end{array}$ & $\begin{array}{c}-0.076 \\
(-1.852)\end{array}$ & $\begin{array}{c}-0.113 \\
(-1.507)\end{array}$ & $\begin{array}{c}-0.126 \\
(-2.358)\end{array}$ & $\begin{array}{c}-0.128 \\
(-2.961)\end{array}$ \\
\hline Secundaria Incompleta & $\begin{array}{c}\mathbf{0 . 0 2 8} \\
(0.346)\end{array}$ & $\begin{array}{c}-0.012 \\
(-0.214)\end{array}$ & $\begin{array}{c}\mathbf{0 . 0 1 5} \\
(0.321)\end{array}$ & $\begin{array}{c}-0.072 \\
(-0.886)\end{array}$ & $\begin{array}{c}-\mathbf{0 . 0 0 8} \\
(-0.139)\end{array}$ & $\begin{array}{c}-0.032 \\
(-0.674)\end{array}$ & $\begin{array}{c}-0.004 \\
(-0.057)\end{array}$ & $\begin{array}{c}-0.123 \\
(-2.228)\end{array}$ & $\begin{array}{c}-0.079 \\
(-1.758)\end{array}$ & $\begin{array}{c}-0.099 \\
(-1.240)\end{array}$ & $\begin{array}{c}-0.178 \\
(-3.095)\end{array}$ & $\begin{array}{c}-0.158 \\
(-3.409)\end{array}$ \\
\hline Secundaria Completa & $\begin{array}{c}-0.212 \\
(-2.513)\end{array}$ & $\begin{array}{c}-0.110 \\
(-1.768)\end{array}$ & $\begin{array}{c}-0.113 \\
(-2.313)\end{array}$ & $\begin{array}{c}-0.283 \\
(-3.361)\end{array}$ & $\begin{array}{c}-0.143 \\
(-2.231)\end{array}$ & $\begin{array}{c}-0.168 \\
(-3.360)\end{array}$ & $\begin{array}{c}-0.282 \\
(-3.512)\end{array}$ & $\begin{array}{c}-0.251 \\
(-4.229)\end{array}$ & $\begin{array}{c}-0.251 \\
(-5.346)\end{array}$ & $\begin{array}{c}-0.321 \\
(-3.915)\end{array}$ & $\begin{array}{c}-0.381 \\
(-6.251)\end{array}$ & $\begin{array}{c}-0.359 \\
(-7.469)\end{array}$ \\
\hline Superior Incompleta & $\begin{array}{c}-0.178 \\
(-1.732)\end{array}$ & $\begin{array}{c}-0.134 \\
(-1.684)\end{array}$ & $\begin{array}{c}-0.103 \\
(-1.673)\end{array}$ & $\begin{array}{l}-0.419 \\
(-4.217)\end{array}$ & $\begin{array}{c}-0.211 \\
(-2.714)\end{array}$ & $\begin{array}{c}-0.271 \\
(-4.503)\end{array}$ & $\begin{array}{c}-0.263 \\
(-2.697)\end{array}$ & $\begin{array}{c}-0.269 \\
(-3.539)\end{array}$ & $\begin{array}{l}-0.246 \\
(-4.196)\end{array}$ & $\begin{array}{l}-0.489 \\
(-5.047)\end{array}$ & $\begin{array}{c}-0.487 \\
(-6.545)\end{array}$ & $\begin{array}{c}-0.497 \\
(-8.560)\end{array}$ \\
\hline Superior Completa & $\begin{array}{c}-0.496 \\
(-4.697)\end{array}$ & $\begin{array}{c}-0.397 \\
(-3.789)\end{array}$ & $\begin{array}{c}-0.347 \\
(-5.004)\end{array}$ & $\begin{array}{c}-0.629 \\
(-6.316)\end{array}$ & $\begin{array}{c}-0.525 \\
(-5.203)\end{array}$ & $\begin{array}{c}-0.473 \\
(-7.155)\end{array}$ & $\begin{array}{c}-0.709 \\
(-7.124)\end{array}$ & $\begin{array}{c}-0.606 \\
(-6.092)\end{array}$ & $\begin{array}{c}-0.620 \\
(-9.503)\end{array}$ & $\begin{array}{c}-0.787 \\
(-8.161)\end{array}$ & $\begin{array}{c}-0.861 \\
(-8.997)\end{array}$ & $\begin{array}{c}-0.797 \\
(-12.760)\end{array}$ \\
\hline Experiencia Potencial & $\begin{array}{c}-0.018 \\
(-3.404)\end{array}$ & $\begin{array}{c}-0.028 \\
(-6.045)\end{array}$ & $\begin{array}{c}-0.025 \\
(-7.275)\end{array}$ & $\begin{array}{c}-0.028 \\
(-5.432)\end{array}$ & $\begin{array}{c}-0.026 \\
(-5.490)\end{array}$ & $\begin{array}{c}-0.028 \\
(-8.315)\end{array}$ & $\begin{array}{c}-0.024 \\
(-4.589)\end{array}$ & $\begin{array}{c}-0.028 \\
(-6.156)\end{array}$ & $\begin{array}{c}-0.028 \\
(-8.374)\end{array}$ & $\begin{array}{c}-0.033 \\
(-6.476)\end{array}$ & $\begin{array}{c}-0.028 \\
(-6.114)\end{array}$ & $\begin{array}{c}-0.032 \\
(-9.718)\end{array}$ \\
\hline Exper. Potencial 2 & $\begin{array}{c}\mathbf{0 . 0 0 0} \\
(1.047)\end{array}$ & $\begin{array}{c}0.001 \\
(5.874)\end{array}$ & $\begin{array}{c}0.000 \\
(5.603)\end{array}$ & $\begin{array}{c}0.000 \\
(2.397)\end{array}$ & $\begin{array}{c}0.001 \\
(5.736)\end{array}$ & $\begin{array}{c}0.000 \\
(6.494)\end{array}$ & $\begin{array}{c}0.000 \\
(1.742)\end{array}$ & $\begin{array}{c}\mathbf{0 . 0 0 1} \\
(5.779)\end{array}$ & $\begin{array}{c}0.000 \\
(6.196)\end{array}$ & $\begin{array}{c}0.000 \\
(3.024)\end{array}$ & $\begin{array}{c}\mathbf{0 . 0 0 1} \\
(5.975)\end{array}$ & $\begin{array}{c}0.000 \\
(7.263)\end{array}$ \\
\hline Profesional & $\begin{array}{c}-\mathbf{- 2 . 4 2 8} \\
(-13.726)\end{array}$ & $\begin{array}{c}-2.249 \\
(-14.778)\end{array}$ & $\begin{array}{c}-2.451 \\
(-21.711)\end{array}$ & $\begin{array}{c}-2.214 \\
(-12.439)\end{array}$ & $\begin{array}{c}-1.776 \\
(-11.466)\end{array}$ & $\begin{array}{c}-\mathbf{2 . 0 4 4} \\
(-17.855)\end{array}$ & $\begin{array}{c}-3.087 \\
(-21.436)\end{array}$ & $\begin{array}{c}-2.635 \\
(-20.046)\end{array}$ & $\begin{array}{c}-2.913 \\
(-30.544)\end{array}$ & $\begin{array}{c}-2.594 \\
(-18.069)\end{array}$ & $\begin{array}{c}-2.003 \\
(-15.892)\end{array}$ & $\begin{array}{c}-2.312 \\
(-24.896)\end{array}$ \\
\hline Calificados & $\begin{array}{l}-2.111 \\
(-14.735)\end{array}$ & $\begin{array}{c}-1.887 \\
-15.595)\end{array}$ & $\begin{array}{c}-2.075 \\
(-23.003)\end{array}$ & $\begin{array}{c}-1.950 \\
(-13.115)\end{array}$ & $\begin{array}{c}-1.671 \\
(-12.721)\end{array}$ & $\begin{array}{c}-1.834 \\
(-18.973)\end{array}$ & $\begin{array}{c}-2.735 \\
(-26.520)\end{array}$ & $\begin{array}{l}-2.194 \\
(-22.802)\end{array}$ & $\begin{array}{c}-2.510 \\
(-36.739)\end{array}$ & $\begin{array}{c}-\mathbf{2 . 3 5 3} \\
(-22.220)\end{array}$ & $\begin{array}{c}-1.851 \\
(-19.081)\end{array}$ & $\begin{array}{c}-2.107 \\
(-30.028)\end{array}$ \\
\hline No Calificado & $\begin{array}{c}-\mathbf{2 . 0 7 9} \\
(-14.320)\end{array}$ & $\begin{array}{c}-1.767 \\
(-14.396)\end{array}$ & $\begin{array}{l}-1.965 \\
(-21.466)\end{array}$ & $\begin{array}{c}-1.833 \\
(-12.114)\end{array}$ & $\begin{array}{l}-1.574 \\
(-11.776)\end{array}$ & $\begin{array}{c}-1.714 \\
(-17.425)\end{array}$ & $\begin{array}{c}-2.709 \\
(-26.295)\end{array}$ & $\begin{array}{l}-2.140 \\
(-21.839)\end{array}$ & $\begin{array}{c}-2.436 \\
(-35.218)\end{array}$ & $\begin{array}{c}-\mathbf{2 . 2 0 1} \\
(-20.767)\end{array}$ & $\begin{array}{c}-\mathbf{1 . 8 2 4} \\
(-18.455)\end{array}$ & $\begin{array}{c}-2.014 \\
(-28.359)\end{array}$ \\
\hline Casado & $\begin{array}{c}-0.060 \\
(-1.543)\end{array}$ & $\begin{array}{c}-0.313 \\
(-8.558)\end{array}$ & $\begin{array}{c}-0.208 \\
(-7.952)\end{array}$ & $\begin{array}{c}-0.037 \\
(-0.987)\end{array}$ & $\begin{array}{c}-0.387 \\
(-10.794)\end{array}$ & $\begin{array}{c}-0.224 \\
(-8.820)\end{array}$ & $\begin{array}{c}-0.076 \\
(-2.006)\end{array}$ & $\begin{array}{c}-0.317 \\
(-8.821)\end{array}$ & $\begin{array}{c}-0.203 \\
(-7.927)\end{array}$ & $\begin{array}{c}-0.041 \\
(-1.113)\end{array}$ & $\begin{array}{c}-0.385 \\
(-10.990)\end{array}$ & $\begin{array}{c}-0.213 \\
(-8.527)\end{array}$ \\
\hline Escuela & $\begin{array}{c}0.099 \\
(1.225)\end{array}$ & $\begin{array}{c}\mathbf{0 . 0 1 1} \\
(0.140)\end{array}$ & $\begin{array}{c}\mathbf{0 . 0 5 1} \\
(0.923)\end{array}$ & $\begin{array}{c}0.044 \\
(0.611)\end{array}$ & $\begin{array}{c}\mathbf{0 . 1 2 5} \\
(1.853)\end{array}$ & $\begin{array}{c}0.102 \\
(2.080)\end{array}$ & $\begin{array}{c}\mathbf{0 . 0 5 2} \\
(0.663)\end{array}$ & $\begin{array}{c}-0.050 \\
(-0.659)\end{array}$ & $\begin{array}{c}0.006 \\
(0.109)\end{array}$ & $\begin{array}{c}\mathbf{0 . 0 1 5} \\
(0.208)\end{array}$ & $\begin{array}{c}0.070 \\
(1.052)\end{array}$ & $\begin{array}{c}\mathbf{0 . 0 5 6} \\
(1.160)\end{array}$ \\
\hline Hijos Solteros & $\begin{array}{c}0.018 \\
(1.639)\end{array}$ & $\begin{array}{c}-0.005 \\
(-0.529)\end{array}$ & $\begin{array}{c}\mathbf{0 . 0 0 2} \\
(0.336)\end{array}$ & $\begin{array}{c}\mathbf{0 . 0 5 5} \\
(5.049)\end{array}$ & $\begin{array}{c}\mathbf{0 . 0 0 2} \\
(0.252)\end{array}$ & $\begin{array}{c}0.022 \\
(3.262)\end{array}$ & $\begin{array}{c}0.017 \\
(1.545)\end{array}$ & $\begin{array}{c}0.000 \\
(0.013)\end{array}$ & $\begin{array}{c}\mathbf{0 . 0 0 6} \\
(0.883)\end{array}$ & $\begin{array}{c}\mathbf{0 . 0 5 5} \\
(5.098)\end{array}$ & $\begin{array}{c}\mathbf{0 . 0 0 4} \\
(0.502)\end{array}$ & $\begin{array}{c}0.023 \\
(3.461)\end{array}$ \\
\hline Primario & $\begin{array}{c}-0.521 \\
(-1.847)\end{array}$ & $\begin{array}{c}-0.131 \\
(-1.116)\end{array}$ & $\begin{array}{c}-0.274 \\
(-2.782)\end{array}$ & $\begin{array}{c}0.296 \\
(1.179)\end{array}$ & $\begin{array}{c}-0.113 \\
(-0.843)\end{array}$ & $\begin{array}{c}-0.131 \\
(-1.189)\end{array}$ & & & & & & \\
\hline Industria & $\begin{array}{c}-0.231 \\
(-2.024)\end{array}$ & $\begin{array}{c}-0.209 \\
(-2.239)\end{array}$ & $\begin{array}{c}-0.256 \\
(-3.637)\end{array}$ & $\begin{array}{c}-0.193 \\
(-1.548)\end{array}$ & $\begin{array}{c}-0.206 \\
(-1.963)\end{array}$ & $\begin{array}{c}-0.237 \\
(-2.999)\end{array}$ & & & & & & \\
\hline Sector Público & $\begin{array}{c}-0.173 \\
(-0.728)\end{array}$ & $\begin{array}{c}-0.345 \\
(-2.279)\end{array}$ & $\begin{array}{c}-0.378 \\
(-3.063)\end{array}$ & $\begin{array}{c}-0.600 \\
(-1.484)\end{array}$ & $\begin{array}{c}-0.363 \\
(-1.966)\end{array}$ & $\begin{array}{c}-0.465 \\
(-2.869)\end{array}$ & & & & & & \\
\hline Construcción & $\begin{array}{c}-0.355 \\
(-1.274)\end{array}$ & $\begin{array}{c}\mathbf{0 . 0 4 5} \\
(0.469)\end{array}$ & $\begin{array}{c}-0.094 \\
(-1.254)\end{array}$ & $\begin{array}{c}-0.091 \\
(-0.393)\end{array}$ & $\begin{array}{c}0.287 \\
(2.753)\end{array}$ & $\begin{array}{c}\mathbf{0 . 1 8 3} \\
(2.295)\end{array}$ & & & & & & \\
\hline Comercio & $\begin{array}{c}-0.459 \\
(-4.184)\end{array}$ & $\begin{array}{c}-0.274 \\
(-2.942)\end{array}$ & $\begin{array}{c}-0.367 \\
(-5.276)\end{array}$ & $\begin{array}{c}-0.298 \\
(-2.554)\end{array}$ & $\begin{array}{c}-0.277 \\
(-2.717)\end{array}$ & $\begin{array}{c}-0.303 \\
(-4.007)\end{array}$ & & & & & & \\
\hline Transporte & $\begin{array}{c}-0.215 \\
(-1.437)\end{array}$ & $\begin{array}{c}-0.267 \\
(-2.671)\end{array}$ & $\begin{array}{c}-0.359 \\
(-4.565)\end{array}$ & $\begin{array}{c}-0.139 \\
(-0.947)\end{array}$ & $\begin{array}{c}-0.195 \\
(-1.806)\end{array}$ & $\begin{array}{c}-0.249 \\
(-2.996)\end{array}$ & & & & & & \\
\hline Servicios Sociales & $\begin{array}{c}-0.791 \\
(-7.389)\end{array}$ & $\begin{array}{c}-0.758 \\
(-7.835)\end{array}$ & $\begin{array}{c}-0.733 \\
(-10.530)\end{array}$ & $\begin{array}{c}-0.582 \\
(-5.059)\end{array}$ & $\begin{array}{c}-0.641 \\
(-6.064)\end{array}$ & $\begin{array}{c}-0.586 \\
(-7.689)\end{array}$ & & & & & & \\
\hline Servicio Doméstico & $\begin{array}{c}-0.384 \\
(-3.107)\end{array}$ & $\begin{array}{c}-0.039 \\
(-0.296)\end{array}$ & $\begin{array}{c}-0.268 \\
(-3.326)\end{array}$ & $\begin{array}{c}-0.243 \\
(-1.888)\end{array}$ & $\begin{array}{c}-0.021 \\
(-0.156)\end{array}$ & $\begin{array}{c}-0.156 \\
(-1.824)\end{array}$ & & & & & & \\
\hline Tamaño 1 & $\begin{array}{l}-0.312 \\
(-3.870)\end{array}$ & $\begin{array}{c}-0.154 \\
(-2.817)\end{array}$ & $\begin{array}{c}-0.184 \\
(-4.105)\end{array}$ & $\begin{array}{c}-0.175 \\
(-2.093)\end{array}$ & $\begin{array}{c}-0.065 \\
(-1.040)\end{array}$ & $\begin{array}{c}-0.084 \\
(-1.695)\end{array}$ & & & & & & \\
\hline Tamaño 2 & $\begin{array}{c}-0.200 \\
(-2.728)\end{array}$ & $\begin{array}{c}-0.269 \\
(-5.077)\end{array}$ & $\begin{array}{c}-0.224 \\
(-5.265)\end{array}$ & $\begin{array}{c}-0.103 \\
(-1.355)\end{array}$ & $\begin{array}{c}-0.117 \\
(-1.982)\end{array}$ & $\begin{array}{c}-0.098 \\
(-2.107)\end{array}$ & & & & & & \\
\hline Tamaño 3 & $\begin{array}{c}-0.227 \\
(-3.086)\end{array}$ & $\begin{array}{c}-0.267 \\
(-4.776)\end{array}$ & $\begin{array}{c}-0.230 \\
(-5.232)\end{array}$ & $\begin{array}{c}-0.009 \\
(-0.122)\end{array}$ & $\begin{array}{c}-0.056 \\
(-0.913)\end{array}$ & $\begin{array}{c}-0.025 \\
(-0.530)\end{array}$ & & & & & & \\
\hline Tamaño 4 & $\begin{array}{c}-0.234 \\
(-2.899)\end{array}$ & $\begin{array}{c}-0.154 \\
(-2.523)\end{array}$ & $\begin{array}{c}-0.173 \\
(-3.594)\end{array}$ & $\begin{array}{c}-0.209 \\
(-2.436)\end{array}$ & $\begin{array}{c}-0.024 \\
(-0.366)\end{array}$ & $\begin{array}{c}-0.096 \\
(-1.837)\end{array}$ & & & & & & \\
\hline Tamaño 5 & $\begin{array}{c}-0.359 \\
(-3.552)\end{array}$ & $\begin{array}{c}-0.081 \\
(-1.156)\end{array}$ & $\begin{array}{c}-0.178 \\
(-3.118)\end{array}$ & $\begin{array}{c}-0.184 \\
(-1.785)\end{array}$ & $\begin{array}{c}-0.168 \\
(-2.081)\end{array}$ & $\begin{array}{c}-0.189 \\
(-3.020)\end{array}$ & & & & & & \\
\hline Tamaño 6 & $\begin{array}{c}-0.175 \\
(-1.393)\end{array}$ & $\begin{array}{c}\mathbf{0 . 1 1 5} \\
(1.382) \\
\end{array}$ & $\begin{array}{c}\mathbf{0 . 0 0 1} \\
(0.016)\end{array}$ & $\begin{array}{c}-0.338 \\
(-2.255)\end{array}$ & $\begin{array}{c}-0.012 \\
(-0.109)\end{array}$ & $\begin{array}{c}-0.142 \\
(-1.650)\end{array}$ & & & & & & \\
\hline $\begin{array}{l}\text { Chi-2 } \\
\text { Obs }\end{array}$ & $\begin{array}{c}1978 \\
11261\end{array}$ & $\begin{array}{c}1551 \\
17984\end{array}$ & $\begin{array}{c}3423 \\
29245\end{array}$ & $\begin{array}{l}1514 \\
9680\end{array}$ & $\begin{array}{c}1535 \\
13917\end{array}$ & $\begin{array}{c}2848 \\
23597 \\
\end{array}$ & $\begin{array}{c}1840 \\
11261\end{array}$ & $\begin{array}{c}1287 \\
17984\end{array}$ & $\begin{array}{c}3102 \\
29245\end{array}$ & $\begin{array}{l}1418 \\
9680\end{array}$ & $\begin{array}{c}1175 \\
13917\end{array}$ & $\begin{array}{c}2459 \\
23597\end{array}$ \\
\hline
\end{tabular}

Fuente: Elaboración propia en base a datos de la EPH. 


\section{Cuadro 16}

Ecuación para la probabilidad de ser un trabajador informal (Modelo Probit) Argentina, 1994-2000

\begin{tabular}{|c|c|c|c|c|c|c|c|c|c|c|c|c|}
\hline & \multicolumn{6}{|c|}{ Ecuación Completa } & \multicolumn{6}{|c|}{ Ecuación de Características Personales } \\
\hline & \multicolumn{3}{|c|}{1994} & \multicolumn{3}{|c|}{2000} & \multicolumn{3}{|c|}{1994} & \multicolumn{3}{|c|}{2000} \\
\hline & Hombre & Mujer & Total & Hombre & Mujer & Total & Hombre & Mujer & Total & Hombre & Mujer & Total \\
\hline Constante & $\begin{array}{c}\mathbf{0 . 3 3 7} \\
(0.597)\end{array}$ & $\begin{array}{c}0.442 \\
(1.086)\end{array}$ & $\begin{array}{c}\mathbf{0 . 4 1 2} \\
(1.256)\end{array}$ & $\begin{array}{c}\mathbf{0 . 9 2 3} \\
(1.972)\end{array}$ & $\begin{array}{c}\mathbf{0 . 4 9 8} \\
(0.989)\end{array}$ & $\begin{array}{c}\mathbf{0 . 6 6 5} \\
(1.980)\end{array}$ & $\begin{array}{c}1.471 \\
(2.948)\end{array}$ & $\begin{array}{c}1.127 \\
(3.060)\end{array}$ & $\begin{array}{c}1.254 \\
(4.243)\end{array}$ & $\begin{array}{c}1.700 \\
(4.128)\end{array}$ & $\begin{array}{c}1.093 \\
(2.337)\end{array}$ & $\begin{array}{c}1.389 \\
(4.604)\end{array}$ \\
\hline Primaria Completa & $\begin{array}{c}-0.106 \\
(-1.363)\end{array}$ & $\begin{array}{c}-0.145 \\
(-2.396)\end{array}$ & $\begin{array}{c}-0.141 \\
(-2.979)\end{array}$ & $\begin{array}{c}-0.144 \\
(-1.533)\end{array}$ & $\begin{array}{c}-0.193 \\
(-2.420)\end{array}$ & $\begin{array}{c}-0.179 \\
(-2.966)\end{array}$ & $\begin{array}{c}-0.357 \\
(-5.007)\end{array}$ & $\begin{array}{l}-0.213 \\
(-3.829)\end{array}$ & $\begin{array}{c}-0.285 \\
(-6.590)\end{array}$ & $\begin{array}{c}-0.227 \\
(-2.537)\end{array}$ & $\begin{array}{l}-0.216 \\
(-2.992)\end{array}$ & $\begin{array}{c}-0.230 \\
(-4.146)\end{array}$ \\
\hline Secundaria Incompleta & $\begin{array}{c}-0.035 \\
(-0.391)\end{array}$ & $\begin{array}{c}-0.191 \\
(-2.849)\end{array}$ & $\begin{array}{c}-0.158 \\
(-2.989)\end{array}$ & $\begin{array}{c}-0.149 \\
(-1.435)\end{array}$ & $\begin{array}{c}-0.287 \\
(-3.410)\end{array}$ & $\begin{array}{c}-0.239 \\
(-3.697)\end{array}$ & $\begin{array}{c}-0.481 \\
(-5.990)\end{array}$ & $\begin{array}{c}-0.320 \\
(-5.235)\end{array}$ & $\begin{array}{c}-0.405 \\
(-8.440)\end{array}$ & $\begin{array}{c}-0.318 \\
(-3.216)\end{array}$ & $\begin{array}{c}-0.361 \\
(-4.748)\end{array}$ & $\begin{array}{c}-0.357 \\
(-6.020)\end{array}$ \\
\hline Secundaria Completa & $\begin{array}{c}-0.382 \\
(-4.016)\end{array}$ & $\begin{array}{c}-0.456 \\
(-6.238)\end{array}$ & $\begin{array}{c}-0.473 \\
(-8.322)\end{array}$ & $\begin{array}{c}-0.386 \\
(-3.636)\end{array}$ & $\begin{array}{c}-0.557 \\
(-6.306)\end{array}$ & $\begin{array}{c}-0.502 \\
(-7.514)\end{array}$ & $\begin{array}{c}-0.998 \\
(-11.842)\end{array}$ & $\begin{array}{c}-0.639 \\
(-9.604)\end{array}$ & $\begin{array}{c}-0.815 \\
(-15.917)\end{array}$ & $\begin{array}{c}-0.682 \\
(-6.811)\end{array}$ & $\begin{array}{c}-0.742 \\
(-9.315)\end{array}$ & $\begin{array}{c}-0.725 \\
(-11.872)\end{array}$ \\
\hline Superior Incompleta & $\begin{array}{c}-0.110 \\
(-0.912)\end{array}$ & $\begin{array}{c}-0.513 \\
(-5.423)\end{array}$ & $\begin{array}{c}-0.402 \\
(-5.504)\end{array}$ & $\begin{array}{c}-0.313 \\
(-2.536)\end{array}$ & $\begin{array}{c}-0.616 \\
(-5.931)\end{array}$ & $\begin{array}{c}-0.517 \\
(-6.610)\end{array}$ & $\begin{array}{c}-0.898 \\
(-8.296)\end{array}$ & $\begin{array}{c}-0.817 \\
(-9.404)\end{array}$ & $\begin{array}{c}-0.886 \\
(-13.346)\end{array}$ & $\begin{array}{c}-0.669 \\
(-5.740)\end{array}$ & $\begin{array}{c}-0.866 \\
(-9.218)\end{array}$ & $\begin{array}{c}-0.805 \\
(-11.236)\end{array}$ \\
\hline Superior Completa & $\begin{array}{c}-0.382 \\
(-3.235)\end{array}$ & $\begin{array}{c}-0.601 \\
(-5.386)\end{array}$ & $\begin{array}{c}-0.570 \\
(-7.451)\end{array}$ & $\begin{array}{c}-0.555 \\
(-4.583)\end{array}$ & $\begin{array}{c}-0.747 \\
(-6.496)\end{array}$ & $\begin{array}{c}-0.680 \\
(-8.504)\end{array}$ & $\begin{array}{c}-1.215 \\
(-11.563)\end{array}$ & $\begin{array}{c}-0.990 \\
(-9.633)\end{array}$ & $\begin{array}{c}-1.150 \\
(-16.704)\end{array}$ & $\begin{array}{c}-0.972 \\
(-8.536)\end{array}$ & $\begin{array}{c}\mathbf{- 1 . 1 1 4} \\
(-10.605)\end{array}$ & $\begin{array}{c}-1.061 \\
(-14.624)\end{array}$ \\
\hline Experiencia Potencial & $\begin{array}{c}-0.023 \\
(-3.574)\end{array}$ & $\begin{array}{c}-0.025 \\
(-4.458)\end{array}$ & $\begin{array}{c}-0.027 \\
(-6.544)\end{array}$ & $\begin{array}{l}-0.010 \\
(-1.570)\end{array}$ & $\begin{array}{c}-0.031 \\
(-4.981)\end{array}$ & $\begin{array}{c}-0.021 \\
(-4.688)\end{array}$ & $\begin{array}{c}-0.030 \\
(-4.996)\end{array}$ & $\begin{array}{c}-0.030 \\
(-5.604)\end{array}$ & $\begin{array}{l}-0.029 \\
(-7.496)\end{array}$ & $\begin{array}{c}-0.011 \\
(-1.800)\end{array}$ & $\begin{array}{c}-0.028 \\
(-4.869)\end{array}$ & $\begin{array}{l}-0.016 \\
(-4.040)\end{array}$ \\
\hline Exper. Potencial 2 & $\begin{array}{c}\mathbf{0 . 0 0 1} \\
(4.502)\end{array}$ & $\begin{array}{c}\mathbf{0 . 0 0 0} \\
(4.143)\end{array}$ & $\begin{array}{c}\mathbf{0 . 0 0 1} \\
(6.438)\end{array}$ & $\begin{array}{c}0.000 \\
(1.784)\end{array}$ & $\begin{array}{c}0.001 \\
(4.294)\end{array}$ & $\begin{array}{c}0.000 \\
(4.123)\end{array}$ & $\begin{array}{c}\mathbf{0 . 0 0 1} \\
(5.114)\end{array}$ & $\begin{array}{c}\mathbf{0 . 0 0 1} \\
(4.982)\end{array}$ & $\begin{array}{c}\mathbf{0 . 0 0 1} \\
(6.931)\end{array}$ & $\begin{array}{c}\mathbf{0 . 0 0 0} \\
(1.862)\end{array}$ & $\begin{array}{c}0.000 \\
(3.939)\end{array}$ & $\begin{array}{c}0.000 \\
(3.584)\end{array}$ \\
\hline Antigüedad & $\begin{array}{c}-0.343 \\
(-22.043)\end{array}$ & $\begin{array}{c}-0.311 \\
(-26.783)\end{array}$ & $\begin{array}{c}-0.320 \\
(-34.691)\end{array}$ & $\begin{array}{c}-0.383 \\
(-24.238)\end{array}$ & $\begin{array}{c}-0.323 \\
(-25.007)\end{array}$ & $\begin{array}{c}-0.347 \\
(-35.105)\end{array}$ & $\begin{array}{c}-0.375 \\
(-26.040)\end{array}$ & $\begin{array}{c}-0.341 \\
(-32.077)\end{array}$ & $\begin{array}{c}-0.354 \\
(-41.762)\end{array}$ & $\begin{array}{c}-0.401 \\
(-26.881)\end{array}$ & $\begin{array}{c}-0.347 \\
(-29.663)\end{array}$ & $\begin{array}{c}-0.369 \\
(-40.488)\end{array}$ \\
\hline Profesional & $\begin{array}{c}-0.111 \\
(-0.207)\end{array}$ & $\begin{array}{c}\mathbf{0 . 2 4 7} \\
(0.642)\end{array}$ & $\begin{array}{c}\mathbf{0 . 1 4 3} \\
(0.460)\end{array}$ & $\begin{array}{c}-\mathbf{0 . 1 8 6} \\
(-0.419)\end{array}$ & $\begin{array}{c}\mathbf{0 . 8 5 0} \\
(1.767)\end{array}$ & $\begin{array}{c}\mathbf{0 . 3 9 1} \\
(1.225)\end{array}$ & $\begin{array}{c}-0.286 \\
(-0.580)\end{array}$ & $\begin{array}{c}-0.009 \\
(-0.025)\end{array}$ & $\begin{array}{c}-0.064 \\
(-0.218)\end{array}$ & $\begin{array}{c}-0.183 \\
(-0.455)\end{array}$ & $\begin{array}{c}\mathbf{0 . 8 5 8} \\
(1.837)\end{array}$ & $\begin{array}{c}0.341 \\
(1.141)\end{array}$ \\
\hline Calificados & $\begin{array}{c}-0.033 \\
(-0.062)\end{array}$ & $\begin{array}{c}0.391 \\
(1.050)\end{array}$ & $\begin{array}{c}\mathbf{0 . 2 3 8} \\
(0.787)\end{array}$ & $\begin{array}{c}-0.386 \\
(-0.885)\end{array}$ & $\begin{array}{c}\mathbf{0 . 5 7 4} \\
(1.215)\end{array}$ & $\begin{array}{c}\mathbf{0 . 1 6 6} \\
(0.532)\end{array}$ & $\begin{array}{c}-0.209 \\
(-0.432)\end{array}$ & $\begin{array}{c}\mathbf{0 . 3 2 0} \\
(0.894)\end{array}$ & $\begin{array}{c}\mathbf{0 . 1 3 6} \\
(0.474)\end{array}$ & $\begin{array}{c}-0.291 \\
(-0.739)\end{array}$ & $\begin{array}{c}\mathbf{0 . 7 8 1} \\
(1.703)\end{array}$ & $\begin{array}{c}\mathbf{0 . 2 6 8} \\
(0.916)\end{array}$ \\
\hline No Calificado & $\begin{array}{l}-0.046 \\
(-0.087)\end{array}$ & $\begin{array}{c}0.430 \\
(1.154)\end{array}$ & $\begin{array}{c}\mathbf{0 . 2 6 0} \\
(0.859)\end{array}$ & $\begin{array}{c}\mathbf{0 . 0 2 7} \\
(0.062)\end{array}$ & $\begin{array}{c}\mathbf{0 . 6 6 9} \\
(1.415)\end{array}$ & $\begin{array}{c}\mathbf{0 . 3 9 4} \\
(1.258)\end{array}$ & $\begin{array}{c}\mathbf{0 . 2 7 2} \\
(0.561)\end{array}$ & $\begin{array}{c}\mathbf{0 . 3 3 1} \\
(0.922)\end{array}$ & $\begin{array}{c}\mathbf{0 . 3 8 7} \\
(1.342)\end{array}$ & $\begin{array}{c}0.557 \\
(1.411)\end{array}$ & $\begin{array}{c}0.927 \\
(2.019)\end{array}$ & $\begin{array}{c}\mathbf{0 . 7 9 4} \\
(2.709)\end{array}$ \\
\hline Casado & $\begin{array}{c}\mathbf{0 . 0 8 0} \\
(1.780)\end{array}$ & $\begin{array}{c}-0.227 \\
(-5.450)\end{array}$ & $\begin{array}{c}-0.090 \\
(-3.005)\end{array}$ & $\begin{array}{c}\mathbf{0 . 0 1 9} \\
(0.437)\end{array}$ & $\begin{array}{c}-0.171 \\
(-3.830)\end{array}$ & $\begin{array}{c}-0.087 \\
(-2.846)\end{array}$ & $\begin{array}{l}\mathbf{0 . 0 0 0} \\
(-0.007)\end{array}$ & $\begin{array}{c}-0.281 \\
(-7.253)\end{array}$ & $\begin{array}{c}-0.170 \\
(-6.165)\end{array}$ & $\begin{array}{c}-0.009 \\
(-0.202)\end{array}$ & $\begin{array}{c}-0.231 \\
(-5.575)\end{array}$ & $\begin{array}{c}-0.153 \\
(-5.372)\end{array}$ \\
\hline Escuela & $\begin{array}{c}\mathbf{0 . 0 5 5} \\
(0.571)\end{array}$ & $\begin{array}{c}0.144 \\
(1.693)\end{array}$ & $\begin{array}{c}\mathbf{0 . 1 0 9} \\
(1.704)\end{array}$ & $\begin{array}{c}\mathbf{0 . 0 8 2} \\
(0.915)\end{array}$ & $\begin{array}{c}\mathbf{0 . 0 0 3} \\
(0.034)\end{array}$ & $\begin{array}{c}\mathbf{0 . 0 4 6} \\
(0.731)\end{array}$ & $\begin{array}{c}\mathbf{0 . 0 5 5} \\
(0.596)\end{array}$ & $\begin{array}{c}\mathbf{0 . 0 8 7} \\
(1.097)\end{array}$ & $\begin{array}{c}\mathbf{0 . 0 8 0} \\
(1.331)\end{array}$ & $\begin{array}{c}\mathbf{0 . 0 7 2} \\
(0.836)\end{array}$ & $\begin{array}{c}-0.084 \\
(-1.021)\end{array}$ & $\begin{array}{c}\mathbf{0 . 0 1 4} \\
(0.233)\end{array}$ \\
\hline Primario & $\begin{array}{c}\mathbf{0 . 5 1 5} \\
(1.340)\end{array}$ & $\begin{array}{c}0.196 \\
(1.255)\end{array}$ & $\begin{array}{c}0.244 \\
(1.806)\end{array}$ & $\begin{array}{c}1.026 \\
(2.519)\end{array}$ & $\begin{array}{c}\mathbf{0 . 4 1 4} \\
(2.397)\end{array}$ & $\begin{array}{c}0.478 \\
(3.273)\end{array}$ & & & & & & \\
\hline Industria & $\begin{array}{c}\mathbf{0 . 5 8 4} \\
(2.953)\end{array}$ & $\begin{array}{c}\mathbf{0 . 1 5 6} \\
(1.185)\end{array}$ & $\begin{array}{c}\mathbf{0 . 2 8 5} \\
(2.619)\end{array}$ & $\begin{array}{c}\mathbf{0 . 8 0 6} \\
(4.444)\end{array}$ & $\begin{array}{c}0.261 \\
(1.885)\end{array}$ & $\begin{array}{c}0.443 \\
(4.066)\end{array}$ & & & & & & \\
\hline Sector Público & $\begin{array}{c}-0.054 \\
(-0.126)\end{array}$ & $\begin{array}{c}-\mathbf{0 . 4 3 8} \\
(-2.000)\end{array}$ & $\begin{array}{c}-0.322 \\
(-1.693)\end{array}$ & $\begin{array}{c}\mathbf{0 . 7 4 8} \\
(1.902)\end{array}$ & $\begin{array}{c}-0.285 \\
(-1.173)\end{array}$ & $\begin{array}{c}-0.041 \\
(-0.206)\end{array}$ & & & & & & \\
\hline Construcción & $\begin{array}{c}\mathbf{0 . 8 0 2} \\
(2.239)\end{array}$ & $\begin{array}{c}\mathbf{0 . 6 7 7} \\
(4.998)\end{array}$ & $\begin{array}{c}\mathbf{0 . 7 5 8} \\
(6.663)\end{array}$ & $\begin{array}{c}\mathbf{0 . 1 0 7} \\
(0.317)\end{array}$ & $\begin{array}{c}\mathbf{0 . 5 8 7} \\
(4.110)\end{array}$ & $\begin{array}{c}\mathbf{0 . 6 4 2} \\
(5.570)\end{array}$ & & & & & & \\
\hline Comercio & $\begin{array}{c}\mathbf{0 . 4 5 5} \\
(2.374)\end{array}$ & $\begin{array}{c}0.237 \\
(1.813)\end{array}$ & $\begin{array}{c}0.299 \\
(2.786)\end{array}$ & $\begin{array}{c}0.491 \\
(2.887)\end{array}$ & $\begin{array}{c}0.192 \\
(1.419)\end{array}$ & $\begin{array}{c}\mathbf{0 . 3 1 2} \\
(2.955)\end{array}$ & & & & & & \\
\hline Transporte & $\begin{array}{c}\mathbf{0 . 7 5 4} \\
(3.327)\end{array}$ & $\begin{array}{c}0.543 \\
(4.022)\end{array}$ & $\begin{array}{c}\mathbf{0 . 6 1 7} \\
(5.467)\end{array}$ & $\begin{array}{c}\mathbf{0 . 7 0 8} \\
(3.565)\end{array}$ & $\begin{array}{c}\mathbf{0 . 6 1 4} \\
(4.391)\end{array}$ & $\begin{array}{c}\mathbf{0 . 6 8 9} \\
(6.200)\end{array}$ & & & & & & \\
\hline Servicios Sociales & $\begin{array}{c}\mathbf{0 . 1 1 9} \\
(0.635)\end{array}$ & $\begin{array}{c}-0.111 \\
(-0.848)\end{array}$ & $\begin{array}{c}-0.035 \\
(-0.327)\end{array}$ & $\begin{array}{c}\mathbf{0 . 3 9 0} \\
(2.334)\end{array}$ & $\begin{array}{c}\mathbf{0 . 1 1 2} \\
(0.823)\end{array}$ & $\begin{array}{c}0.245 \\
(2.347)\end{array}$ & & & & & & \\
\hline Servicio Doméstico & $\begin{array}{c}0.790 \\
(3.808)\end{array}$ & $\begin{array}{c}\mathbf{0 . 6 4 1} \\
(3.342)\end{array}$ & $\begin{array}{c}\mathbf{0 . 6 1 0} \\
(5.028)\end{array}$ & $\begin{array}{c}\mathbf{0 . 8 6 8} \\
(4.498)\end{array}$ & $\begin{array}{c}\mathbf{0 . 6 0 5} \\
(2.792)\end{array}$ & $\begin{array}{c}\mathbf{0 . 6 4 9} \\
(5.093)\end{array}$ & & & & & & \\
\hline Tamaño 1 & $\begin{array}{c}0.590 \\
(5.521)\end{array}$ & $\begin{array}{c}\mathbf{0 . 6 7 1} \\
(7.392)\end{array}$ & $\begin{array}{c}\mathbf{0 . 6 7 5} \\
(10.191)\end{array}$ & $\begin{array}{c}\mathbf{0 . 5 9 5} \\
(4.902)\end{array}$ & $\begin{array}{c}1.144 \\
(9.032)\end{array}$ & $\begin{array}{c}0.953 \\
(11.505)\end{array}$ & & & & & & \\
\hline Tamaño 2 & $\begin{array}{c}0.271 \\
(3.346)\end{array}$ & $\begin{array}{c}\mathbf{0 . 6 3 5} \\
(12.023)\end{array}$ & $\begin{array}{c}\mathbf{0 . 5 3 0} \\
(12.087)\end{array}$ & $\begin{array}{c}\mathbf{0 . 4 5 4} \\
(5.366)\end{array}$ & $\begin{array}{c}0.950 \\
(14.672)\end{array}$ & $\begin{array}{c}\mathbf{0 . 7 7 7} \\
(15.330)\end{array}$ & & & & & & \\
\hline Tamaño 3 & $\begin{array}{c}-0.243 \\
(-3.036)\end{array}$ & $\begin{array}{c}-0.012 \\
(-0.229)\end{array}$ & $\begin{array}{c}-0.068 \\
(-1.545)\end{array}$ & $\begin{array}{c}-0.044 \\
(-0.534)\end{array}$ & $\begin{array}{c}0.213 \\
(3.334)\end{array}$ & $\begin{array}{c}\mathbf{0 . 1 1 8} \\
(2.360)\end{array}$ & & & & & & \\
\hline Tamaño 4 & $\begin{array}{c}-0.749 \\
(-7.466)\end{array}$ & $\begin{array}{c}-0.506 \\
(-7.942)\end{array}$ & $\begin{array}{c}-0.570 \\
(-10.638)\end{array}$ & $\begin{array}{c}-0.466 \\
(-5.016)\end{array}$ & $\begin{array}{c}-0.384 \\
(-5.211)\end{array}$ & $\begin{array}{c}-0.401 \\
(-7.045)\end{array}$ & & & & & & \\
\hline Tamaño 5 & $\begin{array}{c}-0.673 \\
(-5.338)\end{array}$ & $\begin{array}{c}-0.649 \\
(-7.802)\end{array}$ & $\begin{array}{c}-0.645 \\
(-9.314)\end{array}$ & $\begin{array}{c}-0.623 \\
(-5.152)\end{array}$ & $\begin{array}{c}-0.465 \\
(-5.373)\end{array}$ & $\begin{array}{c}-\mathbf{0 . 5 3 8} \\
(-7.722)\end{array}$ & & & & & & \\
\hline Tamaño 6 & $\begin{array}{c}-0.555 \\
(-3.328)\end{array}$ & $\begin{array}{c}-0.625 \\
(-5.423)\end{array}$ & $\begin{array}{c}-0.588 \\
(-6.251)\end{array}$ & $\begin{array}{c}-0.300 \\
(-2.052)\end{array}$ & $\begin{array}{c}-0.558 \\
(-4.311)\end{array}$ & $\begin{array}{c}-0.429 \\
(-4.525)\end{array}$ & & & & & & \\
\hline $\begin{array}{l}\text { Chi-2 } \\
\text { obs }\end{array}$ & $\begin{array}{l}2868 \\
7333 \\
\end{array}$ & $\begin{array}{c}3496 \\
10889 \\
\end{array}$ & $\begin{array}{c}6255 \\
18222 \\
\end{array}$ & $\begin{array}{l}3080 \\
6116 \\
\end{array}$ & $\begin{array}{l}3249 \\
7917 \\
\end{array}$ & $\begin{array}{c}6227 \\
14033 \\
\end{array}$ & $\begin{array}{l}2143 \\
7333 \\
\end{array}$ & $\begin{array}{c}2380 \\
10889 \\
\end{array}$ & $\begin{array}{c}4332 \\
18222 \\
\end{array}$ & $\begin{array}{l}2564 \\
6116 \\
\end{array}$ & $\begin{array}{l}2168 \\
7917 \\
\end{array}$ & $\begin{array}{c}4532 \\
14033 \\
\end{array}$ \\
\hline
\end{tabular}

Fuente: Elaboración propia en base a datos de la EPH. 


\section{Cuadro 17}

Tamaño y estructura de las brechas de informalidad y desempleo

Argentina, 1994 - 2000

\begin{tabular}{|c|c|c|c|c|c|c|c|}
\hline & & Discriminación & Err. Est. & Características & Err. Est. & Resto & Total \\
\hline \multicolumn{8}{|c|}{$\begin{array}{l}\text { Ecuación Completa } \\
\text { Informalidad }\end{array}$} \\
\hline \multirow[t]{2}{*}{1994} & Límite I & 0.009 & $(0.009)$ & -0.003 & $(0.008)$ & 0.013 & 0.019 \\
\hline & Límite II & -0.007 & $(0.010)$ & 0.013 & $(0.006)$ & 0.013 & 0.019 \\
\hline 2000 & Límite I & 0.015 & $(0.014)$ & 0.048 & $(0.015)$ & -0.003 & 0.060 \\
\hline \multicolumn{8}{|c|}{ Desempleo } \\
\hline 1994 & Límite II & 0.027 & $(0.006)$ & -0.001 & $(0.003)$ & 0.005 & 0.031 \\
\hline 2000 & Límite I & 0.035 & $(0.009)$ & -0.020 & $(0.009)$ & 0.005 & 0.020 \\
\hline \multicolumn{8}{|c|}{$\begin{array}{l}\text { Ecuación de Características Personales } \\
\text { Informalidad }\end{array}$} \\
\hline \multicolumn{8}{|c|}{ Desempleo } \\
\hline \multirow[t]{2}{*}{1994} & Límite I & 0.017 & $(0.004)$ & 0.002 & $(0.003)$ & 0.012 & 0.031 \\
\hline & Límite II & 0.014 & $(0.005)$ & 0.007 & $(0.002)$ & 0.010 & 0.031 \\
\hline \multirow[t]{2}{*}{2000} & Límite I & 0.013 & $(0.005)$ & 0.000 & $(0.003)$ & 0.008 & 0.021 \\
\hline & Límite II & 0.012 & $(0.006)$ & 0.002 & $(0.003)$ & 0.007 & 0.021 \\
\hline
\end{tabular}

Fuente: Elaboración propia en base a datos de la EPH. Nota: entre paréntesis se presentan los errores estándar (bootstrap). 


\section{Cuadro 18}

Estructura vertical de la discriminación en desempleo e informalidad Argentina, 1994 - 2000

\begin{tabular}{|c|c|c|c|c|c|c|c|c|c|c|c|c|c|c|c|c|}
\hline \multirow{3}{*}{ Informalidad } & \multicolumn{8}{|c|}{ Modelo Completo } & \multicolumn{8}{|c|}{ Modelo Características Personales } \\
\hline & \multicolumn{4}{|c|}{1994} & \multicolumn{4}{|c|}{2000} & \multicolumn{4}{|c|}{1994} & \multicolumn{4}{|c|}{2000} \\
\hline & $\mathrm{HO}$ & $\mathrm{MU}$ & $\% \mathrm{HO}$ & $\% \mathrm{MU}$ & $\mathrm{HO}$ & $\mathrm{MU}$ & $\% \mathrm{HO}$ & $\% \mathrm{MU}$ & $\mathrm{HO}$ & $\mathrm{MU}$ & $\% \mathrm{HO}$ & $\% \mathrm{MU}$ & $\mathrm{HO}$ & $\mathrm{MU}$ & $\% \mathrm{HO}$ & $\% \mathrm{MU}$ \\
\hline Educación & 0.03 & 0.00 & 16 & 0 & 0.05 & 0.00 & 11 & 0 & 0.00 & 0.04 & 0 & 28 & 0.02 & 0.00 & 6 & 0 \\
\hline Experiencia & 0.02 & 0.00 & 14 & 0 & 0.08 & 0.00 & 17 & 0 & 0.01 & 0.00 & 8 & 0 & 0.07 & 0.00 & 18 & 0 \\
\hline Antigüedad & 0.00 & 0.03 & 0 & 16 & 0.00 & 0.08 & 0 & 18 & 0.00 & 0.03 & 0 & 21 & 0.00 & 0.07 & 0 & 21 \\
\hline Tipo de Trabajador & 0.00 & 0.08 & 0 & 51 & 0.00 & 0.26 & 0 & 63 & 0.00 & 0.08 & 0 & 51 & 0.00 & 0.27 & 0 & 79 \\
\hline Región & 0.03 & 0.00 & 16 & 0 & 0.06 & 0.00 & 14 & 0 & 0.02 & 0.00 & 15 & 0 & 0.05 & 0.00 & 12 & 0 \\
\hline Sector Productivo & 0.04 & 0.00 & 27 & 0 & 0.08 & 0.00 & 19 & 0 & - & - & - & - & - & - & - & - \\
\hline Tamaño de la Firma & 0.00 & 0.03 & 0 & 21 & 0.00 & 0.08 & 0 & 18 & - & - & - & - & - & - & - & - \\
\hline Demografia & 0.04 & 0.00 & 27 & 0 & 0.04 & 0.00 & 10 & 0 & 0.05 & 0.00 & 29 & 0 & 0.06 & 0.00 & 15 & 0 \\
\hline Constante & 0.00 & 0.02 & 0 & 12 & 0.13 & 0.00 & 30 & 0 & 0.08 & 0.00 & 48 & 0 & 0.20 & 0.00 & 49 & 0 \\
\hline Total & 0.16 & 0.16 & 100 & 100 & 0.44 & 0.41 & 100 & 100 & 0.16 & 0.15 & 100 & 100 & 0.39 & 0.35 & 100 & 100 \\
\hline \multirow{3}{*}{ Desempleo } & \multicolumn{8}{|c|}{ Modelo Completo } & \multicolumn{8}{|c|}{ Modelo Características Personales } \\
\hline & \multicolumn{4}{|c|}{1994} & \multicolumn{4}{|c|}{2000} & \multicolumn{4}{|c|}{1994} & \multicolumn{4}{|c|}{2000} \\
\hline & $\mathrm{HO}$ & $\mathrm{MU}$ & $\% \mathrm{HO}$ & $\% \mathrm{MU}$ & $\mathrm{HO}$ & $\mathrm{MU}$ & $\% \mathrm{HO}$ & $\% \mathrm{MU}$ & $\mathrm{HO}$ & $\mathrm{MU}$ & $\% \mathrm{HO}$ & $\% \mathrm{MU}$ & $\mathrm{HO}$ & $\mathrm{MU}$ & $\% \mathrm{HO}$ & $\% \mathrm{MU}$ \\
\hline Educación & 0.00 & 0.00 & 0 & 4 & 0.00 & 0.02 & 0 & 15 & 0.00 & 0.00 & 4 & 0 & 0.01 & 0.00 & 6 & 0 \\
\hline Experiencia & 0.00 & 0.01 & 0 & 7 & 0.00 & 0.04 & 0 & 29 & 0.00 & 0.01 & 0 & 16 & 0.00 & 0.04 & 0 & 32 \\
\hline Tipo de Trabajador & 0.00 & 0.03 & 0 & 48 & 0.00 & 0.05 & 0 & 40 & 0.00 & 0.08 & 0 & 82 & 0.00 & 0.09 & 0 & 65 \\
\hline Región & 0.00 & 0.00 & 0 & 4 & 0.00 & 0.01 & 0 & 4 & 0.00 & 0.00 & 0 & 2 & 0.00 & 0.00 & 0 & 3 \\
\hline Sector Productivo & 0.00 & 0.02 & 0 & 25 & 0.00 & 0.01 & 0 & 5 & - & - & - & - & - & - & - & - \\
\hline Tamaño de la Firma & 0.00 & 0.01 & 0 & 12 & 0.00 & 0.01 & 0 & 7 & - & - & - & - & - & - & - & - \\
\hline Demografía & 0.03 & 0.00 & 29 & 0 & 0.06 & 0.00 & 35 & 0 & 0.03 & 0.00 & 25 & 0 & 0.06 & 0.00 & 39 & 0 \\
\hline Constante & 0.07 & 0.00 & 71 & 0 & 0.11 & 0.00 & 65 & 0 & 0.08 & 0.00 & 71 & 0 & 0.08 & 0.00 & 55 & 0 \\
\hline Total & 0.10 & 0.07 & 100 & 100 & 0.16 & 0.13 & 100 & 100 & 0.11 & 0.09 & 100 & 100 & 0.15 & 0.14 & 100 & 100 \\
\hline
\end{tabular}

Fuente: Elaboración propia en base a datos de la EPH. 


\section{Gráfico 1}

\section{Evolución del ingreso individual mensual y del ingreso horario}

(en pesos constantes de 1997)

Gran Buenos Aires, 1985-2000
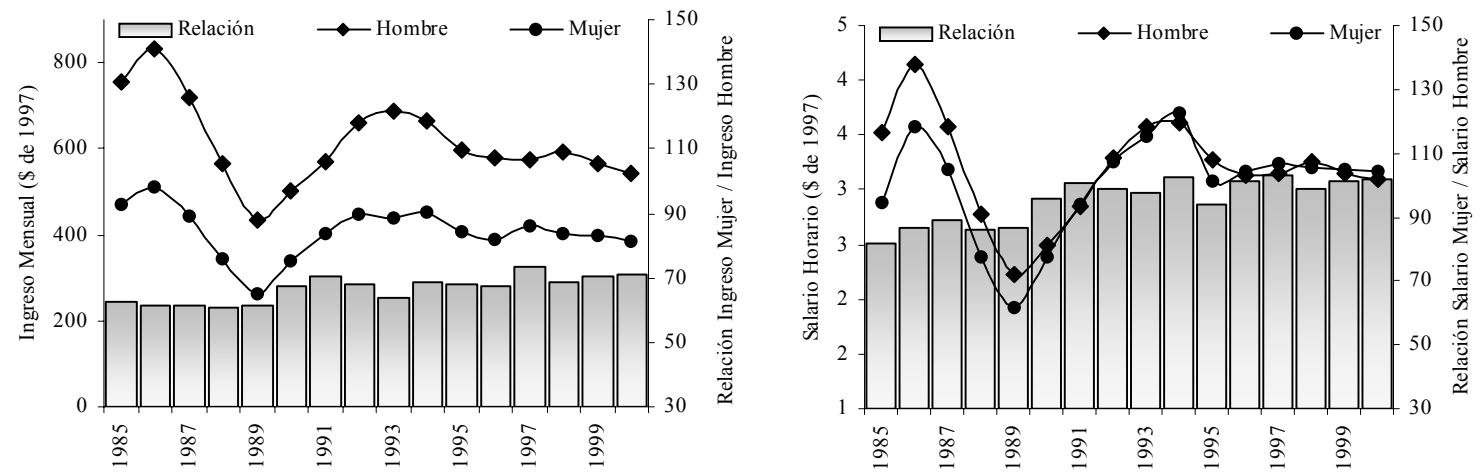

Fuente: Elaboración propia en base a datos de la EPH. Nota: "Relación" indica ingreso promedio (mensual u horario) de las mujeres sobre el ingreso (mensual u horario) de los hombres.

\section{Gráfico 2}

Evolución de la tasa de desempleo y de la tasa de Informalidad Gran Buenos Aires, 1985-2000

A. Tasa de Desempleo

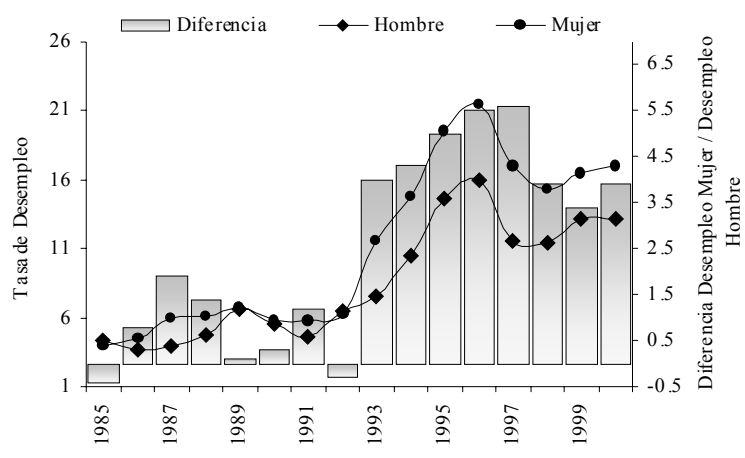

B. Tasa de In formalidad Laboral

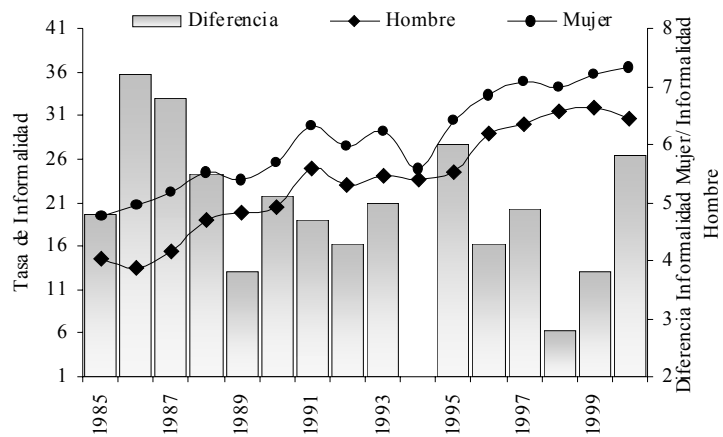

Fuente: Elaboración propia en base a datos de la EPH. Nota: Diferencia indica la tasa de desempleo (o de informalidad) de las mujeres menos la tasa de desempleo (o de informalidad) de los hombres. 


\section{Gráfico 3}

\section{Evolución de la discriminación salarial}

Gran Buenos Aires, 1985-2000

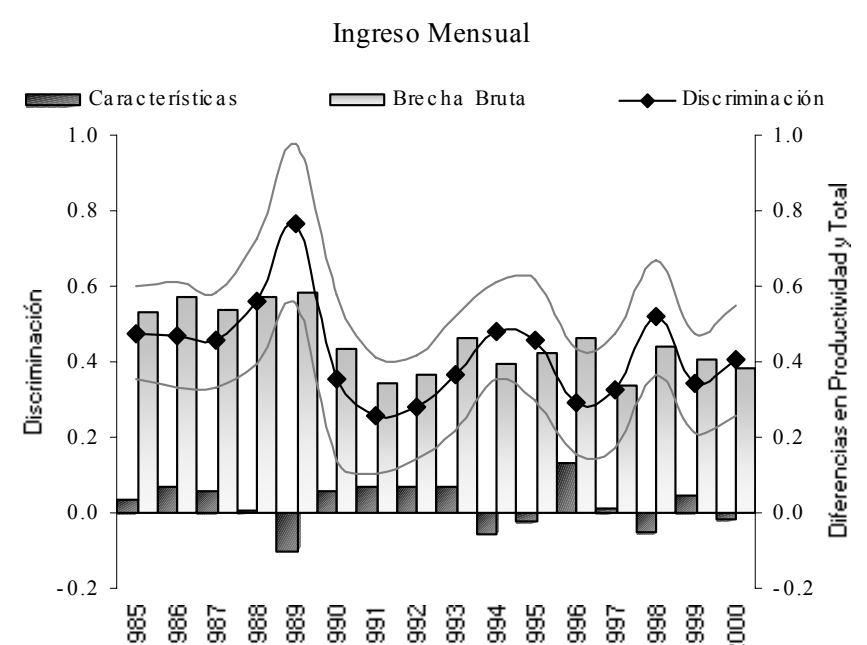

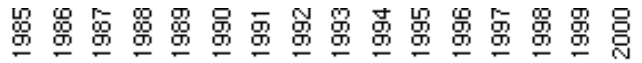

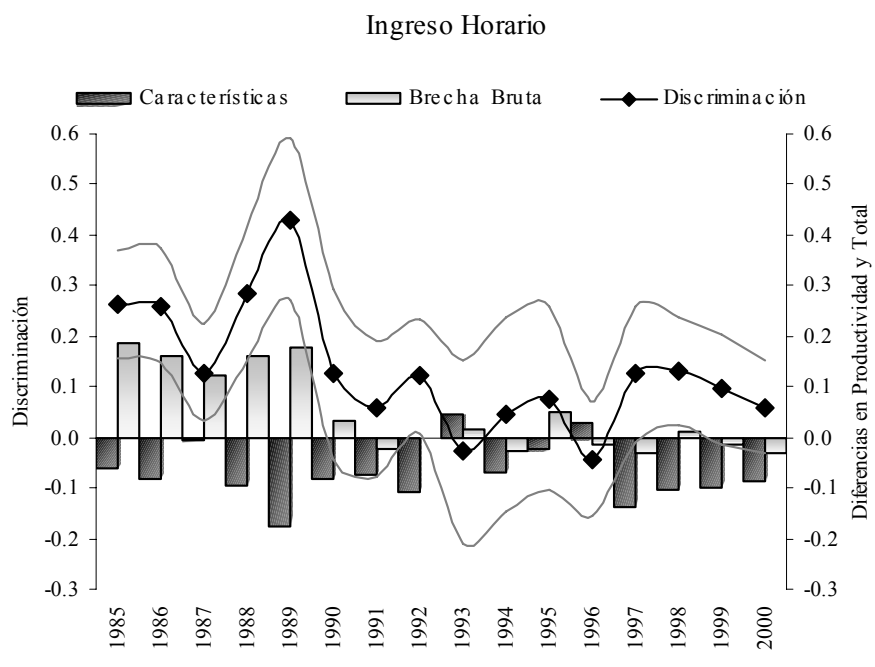

Fuente: Elaboración propia en base a datos de la EPH.

\section{Gráfico 4}

Evolución de la discriminación en desempleo e informalidad

Gran Buenos Aires , 1985-2000

Desempleo

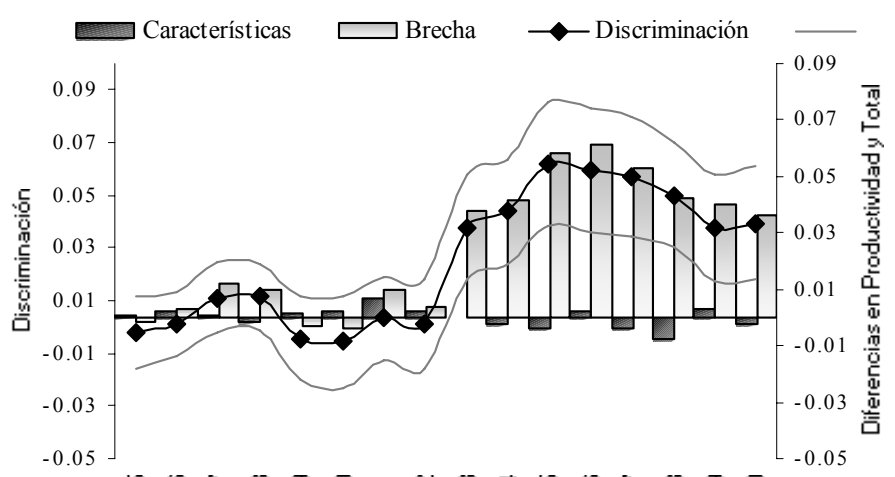

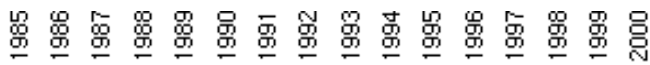

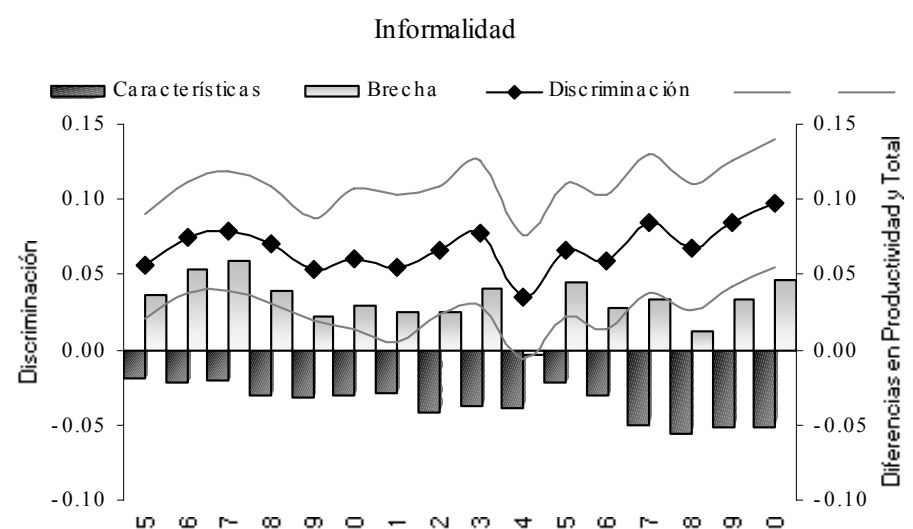

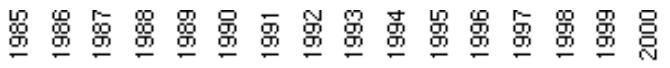

Fuente: Elaboración propia en base a datos de la EPH. Nota: se tomó el promedio simple de las dos descomposiciones posibles. 


\section{Anexo 1}

\begin{tabular}{|c|c|}
\hline Variable & Descripción \\
\hline Ingreso Laboral Horario & Ingreso salarial horario \\
\hline Ingreso Individual & Ingreso Individual en el último mes de referencia \\
\hline Informal & Igual a 1 si el trabajador es informal y a 0 si es informal. \\
\hline Desempleo & Igual a 1 si el trabajador está desempleado y a 0 si está empleado \\
\hline Primaria Incompleta & Primaria incompleta $=1$; En otro caso $=0$ \\
\hline Primaria Completa & Primaria completa =1; En otro caso $=0$ \\
\hline Secundaria Incompleta & Secundaria incompleta $=1 ;$ En otro caso $=0$ \\
\hline Secundaria Completa & Secundaria completa $=1 ;$ En otro caso $=0$ \\
\hline Superior Incompleta & Superior incompleta =1; En otro caso $=0$ \\
\hline Superior Completa & Superior completa $=1 ;$ En otro caso $=0$ \\
\hline Experiencia Potencial & Edad-años de educación-6 \\
\hline Antigüedad & en Años \\
\hline Profesional & $\begin{array}{l}\text { Profesional = 1; En otro caso }=0 \text {. Se consideró profesional a aquellos individuos que declararon trabajar o haber trabajado en algunas de las } \\
\text { siguientes ramas del código CllU-Rev. } 3: 611,801,11,311,301,621,811,21,321,351,631,821,31,331,361,641,831,41,341,371,651,841,51, \\
\text { 381,661,851,61,391,671,861,71,401,681,871,81,411,691,881,91,421,701,891,101,431, } \\
711,901,111,441,721,911,121,451,731,921,131,461,741,931,141,471,751,941,151,481,761,951,161,491,771,961,171,501,781,971,181,511,7 \\
981,191,521,201,531,211,541,221,551,231,561,571,581,591\end{array}$ \\
\hline Calificados & $\begin{array}{l}\text { Calificado = 1; En otro caso }=0 \text {. Se consideró calificado a aquellos individuos que declararon trabajar o haber trabajado en algunas de las } \\
\text { siguientes ramas del código ClIU-Rev. } 3: \\
12,13,22,23,32,33,42,43,52,53,62,63,72,73,82,83,92,93,102,103,112,113,122,123,132,133,142,143,152,153,162,163,172,173,182,183,192,19 \\
202,203,212,213,222,223,232,233,302,303,312,313,322,323,332,333,342,343,352,353,362,363,372,373,382,383,392,393,402,403,412,413,4 \\
423,432,433,442,443,452,453,462,463,472,473,482,483,492,493,502,503,512,513,522,523,532,533,542,543,552,553,562,563,572,573,582,5 \\
592,593,612,613,622,623,632,633,642,643,652,653,662,663,672,673,682,683,692,693,702,703,712,713,722,723,732,733,742,743,752,753,7 \\
763,772,773,782,783,792,793,802,803,812,813,822,823,832,833,842,843,852,853,862,863,872,873,882,883,892,893,902,903,912,913,922,9 \\
932,933,942,943,952,953,962,963,972,973,982,983,\end{array}$ \\
\hline No Calificado & $\begin{array}{l}\text { No calificado }=1 \text {; En otro caso }=0 \text {. Se consideró no calificado a aquellos individuos que declararon trabajar o haber trabajado en algunas de } \\
\text { siguientes ramas del código CllU-Rev. } 3: \\
14,24,34,44,54,64,74,84,94,104,114,124,134,144,154,164,174,184,194,204,214,224,234,304,314,324,334,344,354,364,374,384,394,404,414 \\
4,434,444,454,464,474,484,494,504,514,524,534,544,554,564,574,584,594,614,624,634,644,654,664,674,684,694,704,714,724,734,744,754 \\
4,774,784,794,804,814,824,834,844,854,864,874,884,894,904,914,924,934,944,954,964,974,984,\end{array}$ \\
\hline Otros & Otros $=1 ;$ En otro caso $=0$ \\
\hline Región 1 & Región $1=1$; En otro caso $=0$. La región 1 está compuesta por los siguientes aglomerados urbanos: La Plata, Capital, Conurbano \\
\hline Región 2 & Región $2=1$; En otro caso = 0. La región 2 está compuesta por los siguientes aglomerados urbanos: Rosario, Santa Fe, Paraná, Santa Rosa \\
\hline Región 3 & $\begin{array}{l}\text { Región } 3=1 \text {; En otro caso }=0 \text {. La región } 3 \text { está compuesta por los siguientes aglomerados urbanos: Comodoro Rivadavia, Río Gallegos, Tie } \\
\text { del Fuego, Neuquén, Mendoza }\end{array}$ \\
\hline Región 4 & $\begin{array}{l}\text { Región } 4=1 ; \text {; En otro caso }=0 \text {. La región } 4 \text { está compuesta por los siguientes aglomerados urbanos: Jujuy, Catamarca, Salta, Tucumán, San } \\
\text { Luis, Córdoba, Santiago del Estero, San Juan }\end{array}$ \\
\hline Primario & Primario $=1 ;$ En otro caso = 0. "Primario" indica que el individuo trabaja o ha trabajado por última vez en: actividades primarias \\
\hline Industria & $\begin{array}{l}\text { Industria }=1 ; \text { En otro caso }=0 \text {. "Industria" indica que el individuo trabaja o ha trabajado por última vez en: Ind. De alimentos, bebidas o tabace } \\
\text { Ind. Textiles o del calzado, Ind. De productos químicos o refinación de petróleo, Ind. De productos metálicos, maquinarias y equipos u otras } \\
\text { industrias manufactureras }\end{array}$ \\
\hline Sector Público & $\begin{array}{l}\text { Sector Público }=1 ; \text { En otro caso = } 0 \text {. "Sector Público" indica que el individuo trabaja o ha trabajado por última vez en el sector público nacion } \\
\text { provincial o municipal. }\end{array}$ \\
\hline Construcción & Construcción = 1 ; En otro caso = 0. "Construcción" indica que el individuo trabaja o ha trabajado por última vez en el sector construcción \\
\hline Comercio & $\begin{array}{l}\text { Comercio }=1 ; \text { En otro caso }=0 . \text { "Comercio" indica que el individuo trabaja o ha trabajado por última vez en el comercio al por mayor, al por } \\
\text { menor o en restaurants y hoteles asi como también actividades inmobiliarias o empresariales y de alquiler o en servicios de reparación }\end{array}$ \\
\hline Transporte & $\begin{array}{l}\text { Transporte }=1 ; \text { En otro caso }=0 . \text { "Transporte" indica que el individuo trabaja o ha trabajado por última vez en el sector transporte y servicios } \\
\text { conexos de trasporte y comunicaciones }\end{array}$ \\
\hline Servicios Sociales & $\begin{array}{l}\text { Servicios Sociales }=1 ; \text { En otro caso }=0 \text {. "Servicios Sociales" indica que el individuo trabaja o ha trabajado por última vez en servicios sociale } \\
\text { de salud, servicios comunitarios, servicios de enseñanza o servicios de defensa }\end{array}$ \\
\hline Servicio Doméstico & $\begin{array}{l}\text { Servicio Doméstico }=1 ; \text {; En otro caso = } 0 \text {. "Servicios Doméstico" indica que el individuo trabaja o ha trabajado por última vez en servicios } \\
\text { domésticos u otros servicios personales }\end{array}$ \\
\hline Otros & Otros $=1 ;$ En otro caso $=0$ \\
\hline Tamaño 1 & Tamaño 1 = 1; En otro caso = 0. "Tamaño 1" indica que la persona trabaja o ha trabajado por última vez en un establecimiento con 1 persona \\
\hline Tamaño 2 & Tamaño 2 = 1; En otro caso = 0. "Tamaño 2" indica que la persona trabaja o ha trabajado por última vez en un establecimiento con 2-5 persor \\
\hline Tamaño 3 & $\begin{array}{l}\text { Tamaño } 3=1 ; \text { En otro caso = 0. "Tamaño 3" indica que la persona trabaja o ha trabajado por última vez en un establecimiento con 6-25 } \\
\text { personas }\end{array}$ \\
\hline Tamaño 4 & $\begin{array}{l}\text { Tamaño } 4 \text { = 1; En otro caso = 0. "Tamaño 4" indica que la persona trabaja o ha trabajado por última vez en un establecimiento con 26-100 } \\
\text { personas }\end{array}$ \\
\hline Tamaño 5 & $\begin{array}{l}\text { Tamaño } 5=1 ; \text { En otro caso = 0. "Tamaño 5" indica que la persona trabaja o ha trabajado por última vez en un establecimiento con 101-500 } \\
\text { personas }\end{array}$ \\
\hline Tamaño 6 & $\begin{array}{l}\text { Tamaño } 6=1 \text {; En otro caso = 0. "Tamaño 6" indica que la persona trabaja o ha trabajado por última vez en un establecimiento con más de } 50 \\
\text { personas }\end{array}$ \\
\hline Otros & Otros $=1 ;$ En otro caso $=0$ \\
\hline Edad & Indica la edad del individuo en años \\
\hline Casado & Casado $=1$ (si el individuo está casado o unido) $;$ En otro caso $=0$ \\
\hline Escuela & Escuela = 1 (si el individuo asiste a algún nivel educativo); En otro caso $=0$ \\
\hline Hijos Solteros & Número de hijos solteros \\
\hline Participación & Participación = 1 (si el individuo participa del mercado laboral); En otro caso = 0 \\
\hline
\end{tabular}

